Florida International University

FIU Digital Commons

$7-1-2020$

\title{
3D Architectural Analysis of Neurons, Astrocytes, Vasculature \& Nuclei in the Motor and Somatosensory Murine Cortical Columns
}

Jared Leichner

Department of Biomedical Engineering, Florida International University, jleic001@fiu.edu

Follow this and additional works at: https://digitalcommons.fiu.edu/etd

Part of the Bioimaging and Biomedical Optics Commons, Biotechnology Commons, Computational Neuroscience Commons, Data Science Commons, and the Other Biomedical Engineering and Bioengineering Commons

\section{Recommended Citation}

Leichner, Jared, "3D Architectural Analysis of Neurons, Astrocytes, Vasculature \& Nuclei in the Motor and Somatosensory Murine Cortical Columns" (2020). FIU Electronic Theses and Dissertations. 4457.

https://digitalcommons.fiu.edu/etd/4457

This work is brought to you for free and open access by the University Graduate School at FIU Digital Commons. It has been accepted for inclusion in FIU Electronic Theses and Dissertations by an authorized administrator of FIU Digital Commons. For more information, please contact dcc@fiu.edu. 


\title{
FLORIDA INTERNATIONAL UNIVERSITY
}

Miami, Florida

3D ARCHITECTURAL ANALYSIS OF NEURONS, ASTROCYTES, VASCULATURE \& NUCLEI IN THE MOTOR AND SOMATOSENSORY MURINE CORTICAL COLUMNS

\author{
A dissertation submitted in partial fulfillment of \\ the requirements for the degree of \\ DOCTOR OF PHILOSOPHY
}

in

BIOMEDICAL ENGINEERING

by

Jared Leichner

2020 
To: Dean John L. Volakis

College of Engineering \& Computing

This dissertation, written by Jared Leichner, and entitled 3D Architectural Analysis of Neurons, Astrocytes, Vasculature \& Nuclei in the Motor and Somatosensory Murine Cortical Columns, having been approved in respect to style and intellectual content, is referred to you for judgment.

We have read this dissertation and recommend that it be approved.

$\begin{array}{r}\text { Ranu Jung } \\ \hline \text { Jorge Riera Diaz } \\ \hline \text { Timothy Allen } \\ \hline \text { Wei-Chiang Lin, Major Professor }\end{array}$

Date of Defense: July 1, 2020

The dissertation of Jared Leichner is approved.

Dean John L. Volakis College of Engineering \& Computing

Andrés G. Gil

Vice President for Research and Economic Development and Dean of the University Graduate School

Florida International University, 2020 
(C) Copyright 2020 by Jared Leichner All rights reserved. 


\section{DEDICATION}

I dedicate this Doctoral Dissertation to those who believed in and supported me throughout the preliminary failures, constant optimizations and final success.

First, I thank my parents, who understood the value of combining my MBA studies with a practical doctoral education in engineering so that I can understand not only how to design and produce a critical biotechnology product, but also how to assess the market and understand the best way to strategically bring an entrepreneurial venture to fruition.

Second, I want to thank my wife, who was extraordinarily patient and supportive. After years of waiting for me to return home until late into the night so that I could complete just 'one more experiment,' I can proudly say that the colossal efforts finally bore fruit.

Third, I want to thank my dissertation committee, who had the challenging task of simultaneously reigning in my overambitions, setting achievable but challenging goals in my research, and providing career and life guidance along the way.

Finally, I want to provide sincere thanks to my dissertation advisor and mentor, Dr. Wei-Chiang Lin. Throughout our 9 years of working together, he has definitively shaped my perspective and ability, and transformed me from an idealistic neuroscience enthusiast to a more rigorous and cautioned research scientist. I am overwhelmingly thankful for his patience, as even when my experiments would continue to provide results of inefficient quality, he would still provide me guidance and support as long as he saw that I was still willing to put in the time and effort to better understand the fundamental issues and resolve them. Dr. Lin provided the critical guidance that any research project must start with a complete and thorough understanding of the most fundamental physical phenomena - a lesson that I will carry close to heart the rest of my career. 


\section{ACKNOWLEDGMENTS}

I would like to acknowledge a number of key individuals and groups outside of my dissertation committee which have played critical roles in my research.

Thank you, Dr. Horatiu Vinerean, for your critical support in providing animal tissues that allowed me to carry out these dissertation studies.

Thank you, Dr. Erasmo Perera, as without your patient guidance I would not have been able to collect high-quality brain slices from your vibratome setup.

Thank you, Dr. Timothy Allen, for providing use of your confocal fluorescence microscope on the Modesto Maidique Campus - without which much of this research work would not have been possible.

Thank you, FIU Biomedical Engineering Department, for developing our imaging core facility which provided me the use of confocal fluorescence microscopy on the Engineering campus.

Finally, I want to thank my fellow lab members I have interacted with through group lab meetings during my time in the department who have provided me guidance and support in carrying out my experiments, including Teshaun Francis, Mohamed Almadi, Arash Dadkhah and Zahra Nafar. I also want to thank the BME faculty who have educated me in a variety of important topics during my time as a $\mathrm{PhD}$ Student and the BME student body for their companionship through the journey. 


\section{ABSTRACT OF THE DISSERTATION}

3D ARCHITECTURAL ANALYSIS OF NEURONS, ASTROCYTES, VASCULATURE \& NUCLEI IN THE MOTOR AND SOMATOSENSORY MURINE CORTICAL COLUMNS

by

Jared Leichner

Florida International University, 2020

Miami, Florida

Professor Wei-Chiang Lin, Major Professor

Characterization of the complex cortical structure of the brain at a cellular level is a fundamental goal of neuroscience which can provide a better understanding of both normal function as well as disease state progression. Many challenges exist however when carrying out this form of analysis.

Immunofluorescent staining is a key technique for revealing 3-dimensional structure, but subsequent fluorescence microscopy is limited by the quantity of simultaneous targets that can be labeled and intrinsic lateral and isotropic axial point-spread function (PSF) blurring during the imaging process in a spectral and depth-dependent manner. Even after successful staining, imaging and optical deconvolution, the sheer density of filamentous processes in the neuropil significantly complicates analysis due to the difficulty of separating individual cells in a highly interconnected network of tightly woven cellular arbors.

In order to solve these problems, a variety of methodologies were developed and validated for improved analysis of cortical anatomy. An enhanced immunofluorescent staining and imaging protocol was utilized to precisely locate specific functional regions within brain slices at high magnification and collect four-channel, complete cortical columns. A powerful deconvolution routine was established which collected depth variant PSFs using an optical phantom for image restoration. Fractional volume analysis (FVA) was used to provide preliminary data of the proportions of each stained component in order to statistically characterize the variability within and between the functional regions in a depth-dependent and depth-independent manner. Finally, using machine learning techniques, a supervised learning model was developed that could automatically classify neuronal and astrocytic nuclei within the large cortical column datasets based on perinuclear fluorescence. These annotated nuclei were then used as seed points within 
their corresponding fluorescent channel for cell individualization in a highly interconnected network. For astrocytes, this technique provides the first method for characterization of complex morphology in an automated fashion over large areas without laborious dye filling or manual tracing. 


\section{TABLE OF CONTENTS}

CHAPTER

PAGE

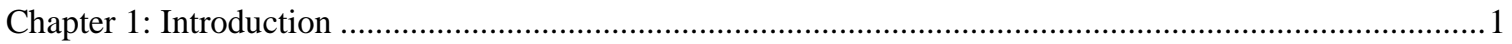

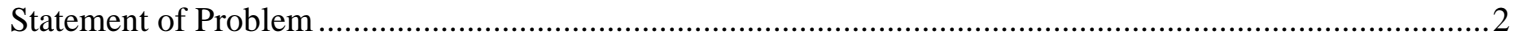

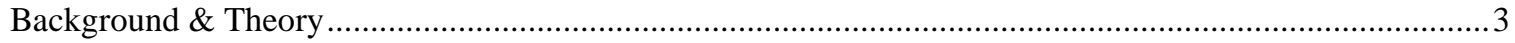

Brain Architectural Study at the Microscopic Level: Technologies................................................... 3

Brain Architectural Study at the Microscopic Level: Applications ......................................................... 7

Brain Architectural Study at the Microscopic Level: Limitations ...........................................................8

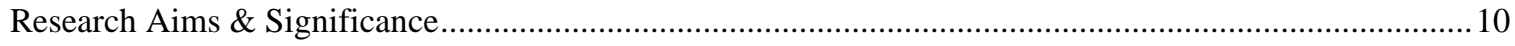

Chapter 2: Acquisition of Neuronal, Astrocytic, Vascular \& Nuclear Data from Murine Cortical Column. 12

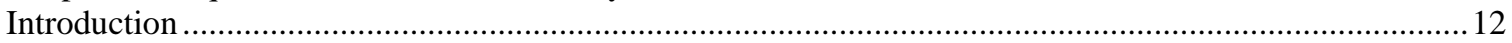

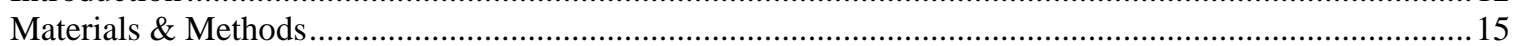

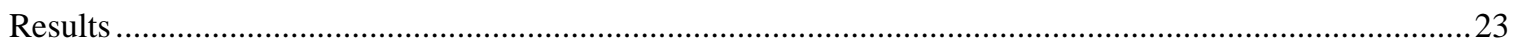

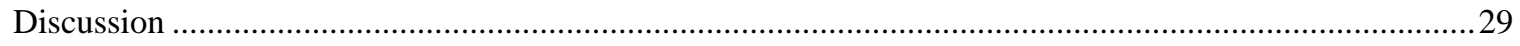

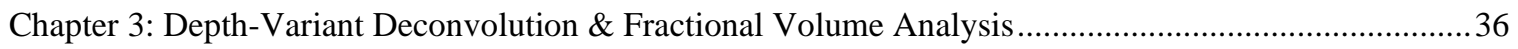

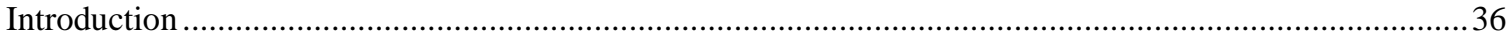

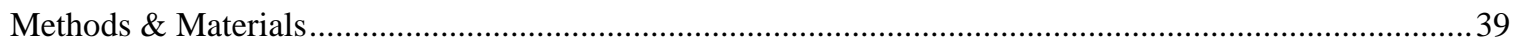

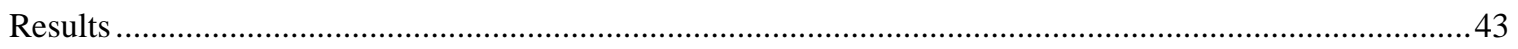

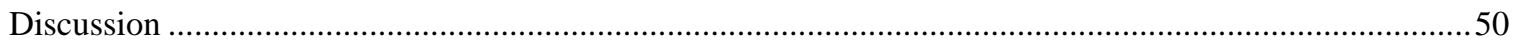

Chapter 4. Automated Nuclei Classification \& Astrocytic Individualization .......................................54

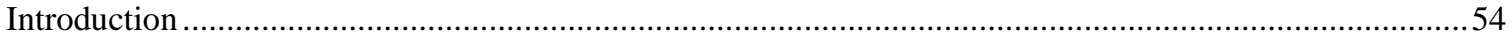

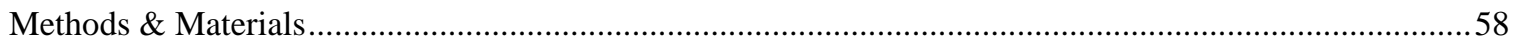

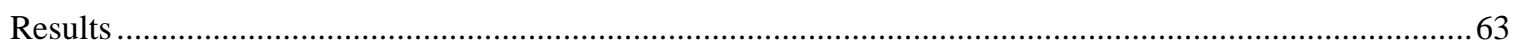

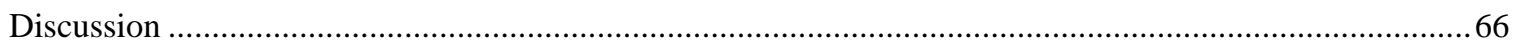

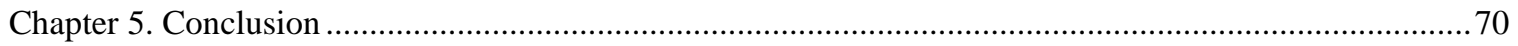

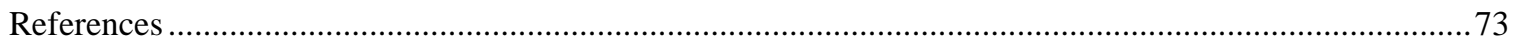

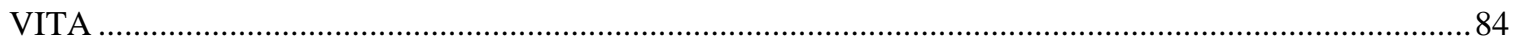




\section{LIST OF TABLES}

TABLE

PAGE

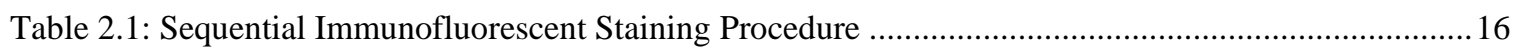

Table 2.2: Materials \& Sources for Experimental Protocol............................................................... 17

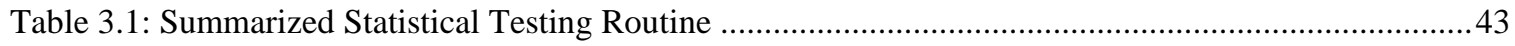

Table 3.2: Summarized Outcomes of Statistical Testing on Volume Fraction Data ................................45

Table 4.1: Sensitivity/Specificity Optimization of Perinuclear Distance Assessment ..............................64

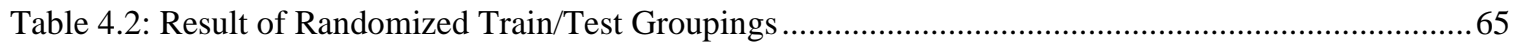




\section{LIST OF FIGURES}

FIGURE

PAGE

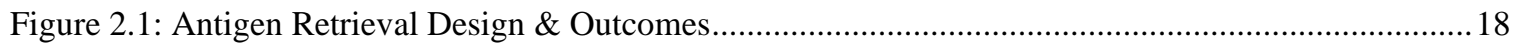

Figure 2.2: Factorial Design for Smi-311 Staining Optimization .............................................................2

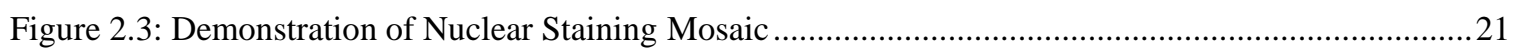

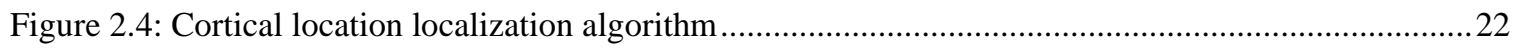

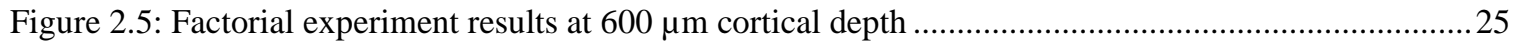

Figure 2.6: Factorial experiment results at $1200 \mu \mathrm{m}$ cortical depth ..........................................................25

Figure 2.7: Four-color staining and cellular architecture at various depths...............................................26

Figure 2.8: Size and scope of a single Z-section in a typical cortical column data cube ...............................2

Figure 2.9: Smi-311 Staining Enhancements Following Stack Contrast Adjustment (SCA) .......................28

Figure 2.10: Volumetric Visualization of Cellular Interconnectivity ..........................................................2 29

Figure 3.1: Depth-Dependent PSF Collection and Sample Chamber Construction ......................................4

Figure 3.2: Impact of Depth-Variant Deconvolution on Data Quality .......................................................4

Figure 3.3: Comparison of Fractional Volume between Regions using Smi-311 (Neuronal) marker............46

Figure 3.4: Comparison of Fractional Volume between Regions using GFAP (Astrocyte) marker .............47

Figure 3.5: Comparison of Fractional Volume between Regions using VWF (Vascular) marker ................48

Figure 3.6: Comparison of Fractional Volume between Regions using Nuclei marker ...............................49

Figure 3.7: Nuclei Density along Cortical Column ..................................................................................

Figure 3.8: Visualization of Interconnectivity of Stained Cellular Markers ..............................................50

Figure 4.1: Complexity of Individual Astrocyte Identification ..............................................................55

Figure 4.2: Demonstration of Perinuclear Parameter Extraction Technique.............................................6

Figure 4.3: Matlab GUI for Nuclei Annotation \& Feature Extraction ....................................................... 61

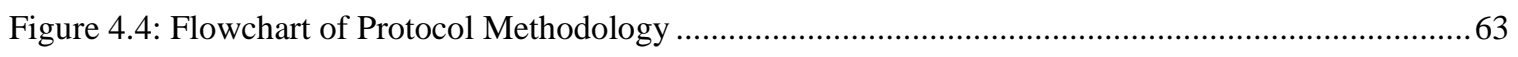

Figure 4.5: Distribution of Nuclei and Hyperplane Development ……..................................................6

Figure 4.6: Astrocyte Individualization and Parameter Extraction ......................................................66 


\section{ABBREVIATIONS AND ACRONYMS}

FVA

GFAP

GUI

M1

PSF

S1BF

VWF
Fractional Volume Analysis

Glial Fibrillary Acidic Protein

Graphic User Interface

Primary Motor Cortex

Point Spread Function

Primary Somatosensory Cortex Barrel Field

Von-Willebrand Factor 


\section{Chapter 1: Introduction}

A thorough understanding of the specific nature of microscopic cellular architecture and heterogeneity of cellular morphology in the neuropil is limited by a lack of multicellular data at the microscopic level. Collection of this data is difficult due to the challenge of imaging such a dense region, the variety of cells involved, and the relative lack of established quantitative metrics in the scientific literature. Due to recent advances in computational hardware, collection of microscopy data over large regions at fine spatial resolutions has become much more efficient - improving the potential of studies of the brain's network architecture. Comprehending the interwoven nature of neurons, astrocytes and vasculature within cortical brain tissue requires a resolution fine enough to identify detailed cell-level morphology and a stitched multi-panel dataset large enough to assess architectural characteristics through

all layers of the cerebral cortex. An enhanced understanding of network architecture can improve understanding of normal brain function, disease state characteristics and can provide insight as to novel therapeutic interventions for resolving diseases.

This dissertation presents a novel combination of immunofluorescent staining targets alongside an optimized staining and confocal imaging methodology for adult Wistar rat cortical brain tissue that highlights four key components simultaneously - neurons, astrocytes, vasculature and nuclei. First, staining of each of these cellular targets is carried out in coronal brain slices from multiple rats. Confocal microscopy is used to subsequently collect 36 cortical columns from the primary motor and somatosensory regions from multiple slices and both hemispheres of each slice. Cortical column datasets encompass large regions $[800 \mu \mathrm{m} \times 3 \mathrm{~mm} \times 70 \mu \mathrm{m}]$ at an extremely fine theoretical voxel resolution $[0.2 \mu \mathrm{m} \times 0.2 \mu \mathrm{m} \times 1 \mu \mathrm{m}]$. Advances are made in large-scale data acquisition and analysis techniques and a rigorous quantitative optimization of staining parameters is performed through a factorial design. Second, depth-variant, accelerated, blind deconvolution is performed to reduce PSF-driven spherical aberration to enhance data quality. Subsequently, fractional volume of each fluorescently stained component is assessed and statistically compared in both a depth-independent as well as a depth-dependent manner. Third, a novel nuclei assignment technique provides rapid and automated categorization of astrocytic and neuronal nuclei 
throughout the cortical column using support vector machine discrimination through perinuclear staining intensity. Astrocyte nuclei classification allows rapid cellular individualization and assessment of morphology.

\section{Statement of Problem}

In order to develop treatment strategies for disease states in the brain, it is critical to understand the specific modifications the disease induces in the complex network architecture. Furthermore, to characterize the various morphological influences of the disease state, it is similarly necessary to first have a firm understanding of the morphology, cellular density and network interconnectivity of normal, undiseased tissue. While macroscopic brain imaging techniques have made tremendous strides in understanding broad network connectivity, they are insufficient for understanding the microscopic alterations in cellular structure and architecture. Microscopic imaging techniques can analyze at these scales, but each of the various approaches provides unique tradeoffs in regard to cost, spatial resolution, field of view and quantity of simultaneously stained targets.

Due to the rapidly shrinking cost of data storage coupled with continued growth in the graphical and computational capabilities of the typical laboratory workstation, it has only recently become feasible to collect extremely large datasets at fine spatial resolutions and then deconvolve them for detailed 3D architectural analysis using advanced software packages such as Bitplane Imaris. Since these capabilities are extremely recent, there is very little presence in the scientific literature of relevant metrics. While researchers have previously provided stunning visualizations of network architecture or have carried out detailed morphological analysis on individual cells or single-cell populations, there is a substantial gap in the current literature regarding how fractional volume and individual cell morphology in the dense neuropil are organized in a region-specific and cortical depth-specific manner.

To contribute to this underrepresented area, this dissertation is unique in its focus on four specific structural cellular markers, the underlying staining and antigen retrieval strategies, the comparison of different functional regions over numerous cortical column datasets in different rats and utilization of 
depth-variant deconvolution and subsequent 3D modeling to extract pertinent quantitative architectural metrics and astrocyte-specific morphological metrics.

\section{Background \& Theory}

Studying the characteristics of normal brain tissue requires an approach which identifies the unique aspects of structural network connectivity, since the fundamental electrical and chemical signaling which underlies cellular communication requires a dynamic collection of cellular nodes operating at multiple hierarchical levels. Furthermore, understanding the complex manifestation of various disease states within brain tissue requires a simultaneous macroscopic and microscopic perspective to understand the local and regional phenomena. Ultimately, the networked connections between various cellular components are the most substantial controllers of brain functionality and are thus suitable targets for characterizing normal and diseased tissue. However, due to the multi-relational interactions between regions on numerous hierarchical scales, any decomposition of the brain network will fundamentally offer an incomplete perspective of the true landscape of the brain (Pessoa, 2014).

\section{Brain Architectural Study at the Microscopic Level: Technologies}

At the microscopic level, the tools and technologies for assessing the network architecture of the brain are vastly different than those at the macroscopic scale. Staining of brain tissue at microscopic scales has been the most accessible method for centuries to provide sufficient contrast to delineate the structure of particular cell types from the surrounding tissue. This practice first began with the work of Camillo Golgi in 1873 , who utilized silver staining and their resulting impregnation into neurons and their processes to provide detailed tracings of dendrites (Golgi, 1873). This and similar staining techniques that rely on immunohistochemistry have been instrumental in revealing the fundamental shape and structure of cell types in the brain. To understand the additional complexity of a 3-dimensional analysis, it is far more suitable to incorporate fluorescent probes - a practice known as immunofluorescent staining.

Early fluorescence microscopy was limited to widefield microscopy, which illuminates a sample and collects fluorescence from all lateral and axial components simultaneously. While the objective used focuses optimally at a precise focal plane, fluorescent signals from out-of-focus regions still reach the 
objective and reduces image clarity. To enhance image quality, a spatial pinhole was introduced to block the out-of-focus light from contributing to the final image, with the resultant technology called confocal microscopy. Using this confocal technique, in conjunction with optical sectioning, it becomes possible to capture entire 3D objects through careful manipulation of the focal volume along the axial dimension (Pawley, 2006). Recently, wide field microscopes have been shown to be capable of this sectioning capability (Cogswell \& Sheppard, 2014), but they are far more limited at this task. While confocal microscopy has seen a variety of incremental improvements in recent decades, four major categories of confocal microscopes remain commercially available today. The first type, used within this dissertation, is the confocal laser scanning microscope, which raster scans the laser along the XY axes to collect the final image on a point-by-point basis. This technique has been widely used to study neuronal morphology for decades (Belichenko et al., 1994). The second, spinning disc confocal microscopy, utilizes a spinning Nipkow-Petran disk which contains hundreds of pinholes - unlike typical confocal techniques which only use a single pinhole. These numerous pinholes sample many points in parallel, enhancing image acquisition speed and reducing phototoxicity (Gräf et al., 2005). Additionally, spinning disc confocal microscopy provides substantial customizability of resolution, brightness and contrast through selection of pinhole size and disc spin speed. While this technology allowed for much faster imaging speeds, the desire for higher spatial resolutions and hence smaller pinholes substantially reduces collected light intensities. To resolve this issue, a third category emerged - dual-spinning disc confocal microscopes. This technique utilizes a second spinning disc, which has a similar array of openings but each containing a micro-lens that is associated with a specific pinhole (Nelson et al., 2010). These micro-lens arrays substantially improve the light intensity in the final image while still allowing for extremely small pinholes to maximize spatial resolution. The final category of confocal microscope, called a programmable array microscope (PAM) utilizes a spatial light modulator to precisely control features of individual pixels, such as their opacity or reflectivity. Placed within the light-path, the modulator can allow for arbitrary illumination and detection patterns (Verveer et al., 1998) and precise photoconversion (Fulwyler et al., 2005) within biological applications. All of these techniques share in common a fundamental advantage over widefield techniques - precise optical sectioning. Optical sectioning with fluorescent probes is valuable due to its compatibility 
with thicker tissue sections than typical immunohistochemistry and its ability to preserve the complex features of tissue components.

While confocal microscopy is the most commonly available optical sectioning technology for most laboratories, other more expensive technologies and exotic instrumentation setups such as multiphoton microscopy and light sheet microscopy can allow for enhanced imaging capabilities. Multi-photon microscopy can achieve better tissue penetration due to the use of near-infrared excitation light and can utilize enhanced resolving power due to the limitation of excitation to a smaller focal volume (Konig, 2000). This is possible due to the use of femtosecond pulsed lasers that can generate a high local photon density at the desired focal point, increasing the likelihood of simultaneous excitation of a fluorophore. The sum of the energy of the arising photons satisfies the transition energy required to cause a fluorescent event for fluorophores located at the overlapped focal point of each photon packet's path (Larson, 2011). This complex process yields several important advantages. First, limiting the elevation of the fluorophore from ground to excited state at the intersected focal point reduces the presence of additional excitation along the path, reducing out-of-focus light and minimizing photobleaching. Second, the necessity of longer wavelength (lower energy) illumination sources to combine to the desired energy level typically has improved depth penetration. Light sheet microscopy decouples the illumination and detection pathways, allowing for far higher imaging speeds and enhanced depth penetration - especially when the sample is optically cleared (Power \& Huisken, 2017). This is typically carried out through focusing a laser beam into a thin sheet using a cylindrical lens. This sheet is then swept through the sample while a perpendicular objective collects the resultant fluorescence signal (Hillman et al., 2019). As the sheet scans through the sample, its thin design only excites fluorescence in a small cross-section, allowing the perpendicular objective to collect the signal within the tissue without fluorescence contribution above or below the focal plane. Within the broader goals of neuroscience research, light sheet microscopy can allow for rapid monitoring of dynamics (e.g. calcium) of living neurons or efficient collection of immunofluorescent data from large areas of fixed, cleared tissue. Through combination of light sheet and multiphoton microscopy, the benefits of each have been combined to allow for extremely rapid, highly penetrating multicolor fluorescence microscopy (Mahou et al., 2014). 
While all of these techniques have tradeoffs, they all share a single fundamental limitation that their resolution cannot exceed the diffraction limit of light. One method to overcome this limitation is through the use of electron microscopy techniques due to their substantially better spatial resolution, which can achieve resolving powers of 1 to $2 \mathrm{~nm}$ in biological specimens under optimal conditions (Cooper, 2000). Electron microscopy techniques, while typically used to generate 2-dimensional images, can be used in synchrony with serial sectioning techniques to generate 3-dimensional volumetric data (Briggman \& Denk, 2006). Furthermore, to provide specific contrast to identify unique macromolecules, antibodies labeled with colloidal gold nanoparticles have been used as contrast agents, akin to how fluorescent secondary antibodies are utilized in fluorescent microscopy (Alberts et al., 2002). However, the high resolution (along with other instrumentation-derived limitations) also serves as a substantial drawback when attempting to derive network architecture metrics of numerous cell types over large spatial regions. Alternative optical methods of exceeding the diffraction limit of light have emerged, collectively known as super-resolution microscopy techniques, which can overcome typical resolution limitations. These are subdivided into two major classes of techniques - deterministic and stochastic (Moerner, 2015). Deterministic techniques take advantage of nonlinear responses to excitation light and include STED (Stimulated Emission Depletion Microscopy) and SSIM (Saturated Structured Illumination Microscopy). Stochastic techniques take advantage of temporal complexity in the resulting fluorescent signal and include PALM (Photoactivated Localization Microscopy) and STORM (Stochastic Optical Reconstruction Microscopy). Techniques which exceed the diffraction limit are exceedingly useful for studying brain networks at the microscopic level, since the tight interwoven nature of cellular filaments requires a spatial resolution fine enough to visualize the fine gaps between them for effective segmentation. Furthermore, sub-micron components such as dendritic spines can be far better represented three-dimensionally when using super-resolution techniques (Sawada et al., 2018).

Even with the widespread availability of optical sectioning, the use of 3-dimensional reconstruction is still rare as compared to the use of 2-dimensional analysis and the development of effective quantitative metrics to analyze the data is even more rarely addressed. This is due to a variety of factors, including the depth dependency of the signal to noise ratio, axial stretching due to the microscope point spread function (which can be heterogeneous throughout the sample due to local refractive index 
variability) and the lack of established network architecture metrics in the scientific literature. To assess network architecture from 3-dimensional data cubes, it is critical to be able to identify connectivity nodes where individual cellular components connect. Furthermore, it is important that the spacing between separate cellular components falls below the spatial resolution of the system after PSF-induced blurring. While this does not necessarily need to involve cells of different types, the power of a network architecture study is dependent not only on the dataset size and spatial resolution, but also the number of independently stained components whose interactions can be reliably characterized 3-dimensionally. Researchers have previously generated excitatory neural connectivity hierarchies in cortical columns of the mouse somatosensory cortex (Lefort et al., 2009), using a combination of electrical recording and neuronal staining. Multi-channel staining of entire cortical columns has similarly been carried out examining neuron/interneuron density (H. S. Meyer et al., 2011), but as with all similar attempts the authors stop short of detailed 3D network architecture quantification, likely due to the substantial complexity and data quality requirements of this type of analysis scheme. Quite possibly, the most similar research project to the dissertation presented here was only just recently published and used a combination of expansion microscopy and light-sheet microscopy to scan a thick cortical column of the primary somatosensory cortex of the mouse cortex at an effective resolution of $60 \times 60 \times 90 \mathrm{~nm}$ (Gao et al., 2019). These researchers were able to use 3D computational modeling techniques to assess a variety of important aspects of their data, such as the subcellular compartment distribution, myelination, dendritic spine density/morphology and morphology of reconstructed neurons.

\section{Brain Architectural Study at the Microscopic Level: Applications}

Each of these varied techniques has allowed researchers to make tremendous strides in understanding the inner workings of the brain. As described previously, the work of Camillo Golgi elucidated the fundamental cellular nature of nervous tissue. In our more modern era, fluorescent techniques have been critical to understand a variety of phenomena, including neurovascular coupling (Uhlirova et al., 2016), the blood-brain barrier (Villaseñor \& Collin, 2017) and astrocytic gap junctions (Nakase et al., 2003). One of the more striking recent developments has emerged with the development of 
'brainbow' technology, where random expression of different ratios of separate fluorescent proteins within individual neurons has allowed for labeling individual cells in hundreds of different 'hues,' making the process of separating adjacent neurons in a dense neuropil a far simpler task - subsequently aiding further study of network connectivity (Weissman \& Pan, 2015). Assessing the anatomical interconnectivity of brain tissue has been achieved with super-resolution techniques (Tønnesen et al., 2018) as well as identification and quantification of synapse locations (S. Park et al., 2018). Electron microscopy has also been able to provide insight as to the neuronal architecture of the normal brain (Briggman \& Denk, 2006) and multi-labeling techniques have provided simultaneous visualization of multiple neuronal populations (X. Zhang et al., 2019). Furthermore, such a high magnification has allowed for direct visualization of the structure of a tripartite synapse (Chung et al., 2015), a phenomenon which would be impossible due to PSF-driven aberrations in optical microscopy.

In addition to studies of normal brains, optical techniques have also proven critical to understand the alterations which occur due to various disease states. For example, fluorescent techniques have helped illuminate the structural and functional characteristics of numerous disease states including epilepsy (Qin et al., 2017), ischemic stroke (Gelderblom et al., 2012), Alzheimer's disease (Shioya et al., 2010) and Parkinson's disease (H.-J. Park et al., 2016). Furthermore, the ability to perform simultaneous staining of multiple targets has proven critical for assessing a variety of complex processes, such as the breakdown of the blood brain barrier during various disease states - including epilepsy (Saunders et al., 2015).

Additionally, super-resolution techniques have been used to further understand a variety of disease states, including Huntington's Disease (Lu et al., 2019) and Alzheimer's (Mlodzianoski et al., 2018). Electron microscopy-based techniques have also proven critical to understand disease states in the brain as well, including assessment of the blood brain barrier under conditions of edema (Hirano et al., 1994) and identification of Tau filament structures in Alzheimer's disease (Fitzpatrick et al., 2017).

\section{Brain Architectural Study at the Microscopic Level: Limitations}

Light microscopy and immunohistochemical techniques have been exceedingly useful in the early history of microscopy to illuminate the structure and components of brain tissue but have a fundamental limitation in their inability to represent complex 3-dimensional morphologies. While immunofluorescence 
techniques combined with confocal optical sectioning allows for 3-dimensional analysis, they exhibit limitations due to intrinsic optical phenomena - including the thickness of tissue sections that can be analyzed (due to optical absorption, spherical aberration and objective working distance) and the maximum number of individual fluorescent channels that can be assessed (typically at most four in current gold standard instrumentation). Incorporation of multiphoton techniques reduce the impact of some of these limitations, but at a significant additional financial cost and risk of cell damage for live-cell studies due to the short, high-energy pulse durations (Tauer, 2002). Damage to live cells is not a concern in this dissertation since only fixed samples are used. Light-sheet microscopy improves the rate of data collection but can be far more complex to align, calibrate and collect the resulting image data (Girkin \& Carvalho, 2018). Super-resolution techniques encompass a broad class of methodologies, each with specific disadvantages. However, examples of typical disadvantages in these techniques include the additional cost and complexity of the imaging system, requirements of specialized fluorescent probes, enhanced phototoxicity due to beam strength and imaging depth / volume limitations.

Regardless of which optical imaging techniques are utilized, resultant network studies all suffer from the same fundamental obstacles. First, heterogeneous brightness levels of different filaments (whether due to inhomogeneous staining, lesser epitope presence or depth-dependent absorption/scattering effects) will limit the accuracy of 3-dimensional reconstructions that rely on pixel threshold values for segmentation. Second, heterogeneous diameter of cellular components will be reconstructed at different accuracies due to interaction effects of the system PSF and the filament diameter. Finally, assessment of network connectivity of components from different fluorescent channels must take into account the channel-specific PSF-driven axial stretch, which complicates the resultant analysis.

Electron microscopy techniques on the other hand, while being able to provide substantial improvements in spatial resolution, suffer from numerous limitations which limit their utility for brain network studies. First, sample thicknesses are typically limited to approximately $200 \mathrm{~nm}$, due to multiple scattering events of the beam limiting reconstruction accuracy. This by itself is a tremendous limitation, since accurate three-dimensional network modeling requires a sample thick enough to provide sufficient cellular information for morphological reconstruction. While serial sectioning techniques are available that can provide 3-dimensional reconstruction, they are time-consuming and laborious in nature (Hoffpauir et 
al., 2007). Furthermore, while separate labeling of individual cell types is a well-established method in optical techniques, this technique is available but still in its infancy when applied to electron microscopy (X. Zhang et al., 2019). Additionally, while optical techniques have the capability to be used on living tissue, the processing steps of typical electron microscopy coupled with the magnitude of the electron beam dose make the use of this technique on live tissue unlikely (de Jonge \& Peckys, 2016).

\section{Research Aims \& Significance}

This dissertation was structured around four unique specific aims.

Specific Aim 1: Develop an optimized immunofluorescent staining and imaging protocol which is capable of acquiring entire cortical columns from the Primary Motor (M1) and Primary Somatosensory Barrel-Field

\section{$\underline{(\mathrm{S} 1 \mathrm{BF}) \text { of the Rat Brain. }}$}

Significance:

Simultaneous staining of four fluorescent channels and collection of data across an entire cortical column are both rare, but until now have not yet been carried out simultaneously. Furthermore, methodological advancements in ROI-identification, mosaic scanning and subsequent alignment is presented. Additionally, the use of relatively thick $(70 \mu \mathrm{m})$ brain slices in conjunction with a specific focus on neuronal, astrocytic and vascular processes allows for a unique perspective of the architecture of these critical cortical components. Finally, a thorough factorial experiment is utilized to optimize the staining protocol through unbiased quantitative analysis of resultant image contrast.

Specific Aim 2: Enhance the morphological accuracy of the collected data using a deconvolution routine that takes into account the spatially and spectrally varying PSF, and with the resultant data carry out detailed analysis of fractional volume distributions of volumetrically modeled cellular components. Significance:

A novel, low-cost methodology for multi-channel experimental PSF collection at sparse depths is presented. Subsequent deconvolution substantially enhances data quality by reducing axial stretch which results from spherical aberration - an intrinsic and unavoidable consequence of imaging deep into tissue. Furthermore, the volumetric density of the components of each fluorescent channel in 50 micron 
increments along the cortical column is assessed. Subsequent statistical analysis provides a clear indication of within-brain, between-brain and between-functional region differences, in both a depth-dependent and depth-independent manner.

Specific Aim 3: Create an automated routine for identifying cell nuclei which belong to astrocytes \& neurons and optimize the underlying decision algorithm to maximize sensitivity and specificity.

\section{Significance:}

In order to differentiate adjacent cells within dense interconnected networks and perform morphological analysis in the neuropil, it is necessary to be able to identify the nuclei of each cell of interest so that it can be used as a seed point for cell individualization and morphological analysis. To accomplish this, a novel technique for assigning nuclei based on perinuclear staining density of

fluorescently stained somas is generated and applied to neurons and astrocytes. Subsequent assessment of sensitivity and specificity demonstrate the efficiency of the protocol, which replaces laborious manual tracing and dye-filling techniques.

Specific Aim 4: Leverage identification of astrocytic cell nuclei to automate the process of cell individualization and morphological extraction.

\section{Significance:}

Heterogeneity of astrocyte morphology is a common theme in the literature, as well as the substantial knowledge gap regarding the underlying variation. This is primarily due to the current techniques available to assess morphology, which require either manual tracing or dye-filling, both of which are tedious and poorly suited for collection of large amounts of morphological data. Here, cell nuclei identification tools developed previously are leveraged to create a powerful methodology for rapid astrocyte morphological analysis in large multichannel fluorescent datasets. 
Chapter 2. Acquisition of Neuronal, Astrocytic, Vascular \& Nuclear Data from Murine Cortical Column

\section{Introduction}

Staining of brain tissue has been the most accessible method for centuries in order to provide sufficient contrast to delineate the structure of particular cell types from the surrounding tissue. This practice first began with the work of Camillo Golgi in 1873, who utilized silver staining and their resulting impregnation into neurons and their processes to provide detailed tracings of dendrites (Golgi, 1873). This and similar staining techniques that rely on immunohistochemistry have been instrumental in revealing the fundamental shape and structure of cell types in the brain but have a fundamental limitation in their inability to represent complex 3-dimensional morphologies. Despite this limitation, these techniques have helped illuminate the structural and functional characteristics of numerous disease states, including epilepsy (Qin et al., 2017), ischemic stroke (Gelderblom et al., 2012), Alzheimer's disease (Shioya et al., 2010) and Parkinson's disease (H.-J. Park et al., 2016).

To understand the additional complexity of a 3-dimensional analysis, it is far more suitable to utilize immunofluorescent staining. Modern immunofluorescent techniques emerge from the foundational work of Dr. Albert Coons, who developed the technique of Fluorescein labeling of immunoglobulin preparations (Beutner, 2003). Using this technique, in accordance with optical sectioning, it becomes possible to capture entire 3D objects through careful manipulation of the focal volume along the axial dimension. While this is typically performed using confocal microscopy methods, wide field microscopes are also capable of this sectioning capability (Cogswell \& Sheppard, 2014). This technique allows for the use of thicker tissue sections than typical immunohistochemistry and preserves the complex connectivity of tissue components. The use of immunofluorescence carries limitations due to intrinsic optical phenomena, including the thickness of tissue sections that can be analyzed (due to optical absorption, spherical aberration and objective working distance) and the maximum number of individual fluorescent channels that can be assessed (typically at most four in current gold standard instrumentation). Spectral signal analysis provides the capability to go beyond four simultaneous channels, but spectral scanning will be far slower, since typical confocal microscopy requires 2-8 microseconds per pixel (Brown et al., 2008) while 
spectral scanning can take approximately 2 milliseconds per pixel (Strasser et al., 2019) - a 1000-fold difference in speed. Due to this, spectral scanning would not be compatible with the large spatial regions scanned in this study due to the substantial increase in imaging time over the $24-48$ hours the scan already requires.

While confocal microscopy is the most commonly available optical sectioning technology for most laboratories, other more expensive technologies and exotic instrumentation setups such as multiphoton microscopy and light sheet microscopy can allow for enhanced imaging capabilities. Multi-photon microscopy is capable of achieving better tissue penetration due to near-infrared excitation light as well as enhanced resolving power due to the limitation of excitation to a smaller focal volume (Konig, 2000). Light sheet microscopy decouples the illumination and detection pathways, allowing for far higher imaging speeds, and enhanced depth penetration - especially when the sample is optically cleared (Power \& Huisken, 2017). Despite the advantages of these advanced methodologies, a 70 micron thick sample assessed using laser scanning confocal microscopy is more than sufficient to examine the interplay of cells and their filamentous processes throughout the thickness of the sample.

Although the developed combination of immunofluorescence staining targets is novel, immunofluorescence with neural markers have been used to accomplish a wide variety of research objectives, including assessments of neurovascular coupling (McCaslin et al., 2011), Parkinson's disease (Reeve et al., 2018), neuroinflammation (Kälin et al., 2015) (Cerbai et al., 2012), Alzheimer's Disease (Kimbrough et al., 2015), vascular connectivity (Wu et al., 2014), tissue clearing strategies (Yang et al., 2014) and axonal process tracing (Gong et al., 2013). However, the use of 3-dimensional reconstruction is still rare as compared to the use of 2-dimensional analysis and the development of effective quantitative metrics to analyze the data is even more rarely addressed. This is due to a variety of factors, including the depth dependency of the signal to noise ratio and axial stretching due to the microscope point spread function.

Throughout this chapter, the term 'cortical column' is not referring to a 'functional' column as for example the 'barrels' in the somatosensory cortex. Instead, it refers to the shape of the collected data cube, since the length of it (from cortical surface to grey/white interface) is far longer than the width of it, creating a data cube with a columnar shape. In the existing literature, examples of full cortical column 
staining are very rare and typically only stain a single cell type at a time, whether neuron (H. S. Meyer et al., 2011), astrocyte (Eilam et al., 2016) or vasculature (Kobat et al., 2011). The rarity of this methodology is due to the inherent complexity of the multi-panel mosaic stitching techniques required, which combine automated-stage microscopy coupled with large-volume image stitching. While many modern labs balance the capture of a large area of interest with a magnification/spatial resolution high enough to accurately identify the finest cellular filaments, it is still relatively uncommon. The capability of identifying these individual filaments is critical for understand the network connectivity throughout the region. Finally, while a single-target strategy can be useful in answering particular research questions, it fails to reveal the nature of the complex network architecture that connects various cell types together.

Compared to staining of a single target, simultaneous immunofluorescence of four separate structural targets is substantially superior due to its unique ability to assess multi-component connectivity. Four-color imaging has been reported as early as the 1990s (Ferri et al., 1997) and has been used to characterize respiratory diseases in single neurons (Grünewald et al., 2014), oxidative phosphorylation defects in muscle fibers (Rocha et al., 2015) and mitochondrial abnormalities in individual osteoblasts (Dobson et al., 2016). The use of simultaneous targets allows for analysis of colocalization and proximity of epitopes of interest, which can yield substantial insight as to the connectivity of the markers analyzed. However, most existing scientific literature utilizing this technique only uses structural markers for one or two of the stained components, and functional markers for the remainder. In contrast, the combination of structural staining targets used in this dissertation is novel and critical for understanding various aspects of cortical network architecture.

The choice of four structural markers provides an opportunity to study the simultaneous interrelation of cellular, neuronal, astrocytic and vascular distributions. For example, this protocol can allow a better understanding of the structural coupling of these cellular components. However, it is important to recognize that confocal microscopy does not provide the spatial resolution to identify tripartite synapses, as the intrinsic blurring due to the microscope's point spread function prevents accurate discrimination of close filament proximity versus actual filament contact. The addition of the nuclear stain provides critical validation that the fluorescently stained structures are individual cells and not artifacts. Overall, it is evident that the use of this simultaneous analysis has an important emergent property and is 
appropriate for better understanding the spatial distributions of the dense filamentous structure of these

critical cellular components. It has been well established that much of the complex interconnectivity of the brain relies on the dynamic connections between these three components (Xue et al., 2013) (Adriani et al., 2017) (Bélanger et al., 2011), and understanding their proximity is a valuable tool for identifying underlying morphological metrics (Tsai et al., 2009). Additionally, the development of accurate brain models or quantitative metrics for model inputs within the field of computational neuroscience relies upon recreation of as much of native tissue structure as possible and the representation of these three cell types three-dimensionally reflect some of the most prominent features of brain tissue.

Thus, this protocol has substantial advantages, as it captures entire cortical columns, threedimensionally, at fine spatial resolutions and simultaneously identifies four critical components of interest. By collecting cortical columns from distinct cortical zones, this protocol becomes capable of yielding both region-specific data as well as cortical layer-specific data. This emerges naturally from the large regions of interest analyzed, which provides the potential capability of determining the fundamental 'building block' or 'repeating unit' that composes each functional brain region. Furthermore, this protocol identifies a series of incremental methodological improvements which will be important for researchers attempting a similar challenge.

\section{Materials \& Methods}

Three adult male Wistar Rats (16-20 weeks) were sacrificed through carbon dioxide euthanasia and then rapidly decapitated. The skull plate was carefully removed and the brain was placed into a solution of 10\% Buffered Formalin (Fisher Scientific, SF99-20) and placed at 4 Celsius. While intracardiac perfusion can provide an improved method of fixation, it was not available for this study and was not a substantial concern since deeper regions of the brain were not characterized. After 48 hours of refrigeration, the brain underwent multiple PBS washes and was switched into a solution of PBS/0.05\% Sodium Azide for storage at 4 degrees Celsius. To generate coronal slices, the brain was placed into an acrylic mold and the cerebellum was removed. The rear surface of the brain was then glued to the vibratome (Leica, VT 1000S) stage and the entire brain was cut into 70 micron slices beginning at the 
olfactory bulb (Cutting Speed: $0.175 \mathrm{~mm} / \mathrm{s}$, Vibration: $80 \mathrm{~Hz}$ ). Each section was photographed and section-specific landmarks were used to map sections to a rat brain atlas (Paxinos \& Watson, 2007). The convergence or divergence of specific structural features of the brain between both hemispheres was utilized to identify the corresponding image of the atlas for each brain slice. From each rat brain, three coronal sections located at the center of the M1 region and three coronal sections located at the center of the S1BF region were collected, encompassing six coronal slices per brain and eighteen in total over the three brains. Subsequent imaging was performed for a cortical column on each hemisphere, providing thirty-six full cortical columns over the entire study. Sections were stored in PBS/0.05\% Sodium Azide in a multi-well plate until immunofluorescent staining occurred.

Immunofluorescent staining utilized Smi-311 (Pan-Neuronal Neurofilament Marker) as the marker for neuronal dendrites, GFAP (Glial Fibrillary Acidic Protein) as the marker for astrocytes, VWF (Von Willebrand Factor) as the marker for vascular endothelium and a commercial staining product called NucRed Dead to fluorescently stain nuclei. Due to the use of two different animal origin secondary antibodies, the markers were sequentially (instead of simultaneously) stained, since simultaneous staining created substantial cross-reactivity between the Smi-311 pAb and VWF sAb. The complete staining protocol is highlighted in Table 2.1 and a list of materials and sources is provided in Table 2.2.

\begin{tabular}{|c|c|c|}
\hline Incubation Solution & Solution Composition & Incubation Time \\
\hline \multicolumn{3}{|c|}{ Step One: Staining of Von-Willebrand Factor } \\
\hline Donkey Blocking Buffer & $\begin{array}{l}\text { 10\% Donkey Serum, 2\% Triton X-100, 0.1\% Cold Water Fish } \\
\text { Gelatin in PBS }\end{array}$ & 12 hours \\
\hline (3x) PBS Wash & PBS & 6 hours each \\
\hline VWF Primary Antibody & 1:50 Sheep VWF, $2 \%$ Triton X-100, $1 \%$ BSA in PBS & 48 hours \\
\hline (3x) PBS Wash & PBS & 6 hours each \\
\hline VWF Primary Antibody & $\begin{array}{l}\text { 1:250 Preadsorbed Donkey Anti-Sheep TRITC, } \\
2 \% \text { Triton X-100, } 1 \% \text { BSA in PBS }\end{array}$ & 48 hours \\
\hline (3x) PBS Wash & PBS & 6 hours each \\
\hline \multicolumn{3}{|c|}{ Step Two: Staining of Smi-311 / GFAP } \\
\hline Goat Blocking Buffer & $\begin{array}{l}10 \% \text { Goat Serum, } 2 \% \text { Triton X-100, } 0.1 \% \text { Cold Water Fish } \\
\text { Gelatin in PBS }\end{array}$ & 12 hours \\
\hline (3x) PBS Wash & PBS & 6 hours each \\
\hline $\begin{array}{l}\text { Smi-311/GFAP Primary } \\
\text { Antibody }\end{array}$ & $\begin{array}{l}\text { 1:100 Mouse Smi-311, } 1: 10 \text { Rabbit GFAP, } \\
2 \% \text { Triton } X-100,1 \% \text { BSA in PBS }\end{array}$ & 48 hours \\
\hline (3x) PBS Wash & PBS & 6 hours each \\
\hline $\begin{array}{l}\text { Smi-311/GFAP Secondary } \\
\text { Antibody }\end{array}$ & $\begin{array}{l}\text { 1:20 Goat anti-mouse BV421, 1:50 Goat anti-rabbit FITC, } 2 \% \\
\text { Triton X-100, } 1 \% \text { BSA in PBS }\end{array}$ & 48 hours \\
\hline (3x) PBS Wash & PBS & 6 hours each \\
\hline \multicolumn{3}{|l|}{ Step Three: Nuclear Staining } \\
\hline Nuclear Stain Buffer & 2 drops of NucRedDead solution in $500 \mathrm{uL}$ of PBS & 15 minutes \\
\hline (3x) PBS Wash & PBS & 5 minutes each \\
\hline
\end{tabular}




\begin{tabular}{|c|c|c|c|}
\hline \multicolumn{2}{|c|}{ Chemical Name } & Manufacturer & Catalogue Number \\
\hline \multicolumn{4}{|c|}{ Antigen Retrieval Materials } \\
\hline Citric Acid & & Sigma Aldrich & $\mathrm{C} 2404$ \\
\hline \multicolumn{4}{|c|}{ Blocking \& Antibody Dilution Buffer Materials } \\
\hline \multicolumn{2}{|l|}{ Tween-20 } & Promega & $\mathrm{H} 5152$ \\
\hline \multicolumn{2}{|l|}{ Donkey Serum } & Sigma Aldrich & D9663 \\
\hline \multicolumn{2}{|l|}{ Goat Serum } & Sigma Aldrich & G-9023 \\
\hline \multicolumn{2}{|l|}{ Triton $\mathrm{X}-100$} & Sigma Aldrich & $\mathrm{X} 100$ \\
\hline \multicolumn{2}{|c|}{ Bovine Serum Albumin } & Sigma Aldrich & A7030 \\
\hline \multicolumn{2}{|c|}{ Cold Water Fish Gelatin } & Sigma Aldrich & G7041 \\
\hline \multicolumn{2}{|l|}{ PBS } & Sigma Aldrich & P5493 \\
\hline \multicolumn{4}{|c|}{ Tissue Slice Mounting Materials } \\
\hline \multicolumn{2}{|l|}{ Glycerol } & Sigma Aldrich & G9012 \\
\hline \multicolumn{4}{|c|}{ Nuclear Staining Materials } \\
\hline \multicolumn{2}{|l|}{ NucRed Dead } & Life Technologies & R37113 \\
\hline \multicolumn{4}{|c|}{ Neuronal (Smi-311) Antibodies } \\
\hline Primary $\mathrm{Ab}$ & Mouse Smi-311 & Biolegend & Smi-311R \\
\hline Secondary $\mathrm{Ab}$ & $\begin{array}{l}\text { Goat anti-Mouse BV421 } \\
\text { (Preadsorbed) }\end{array}$ & Biolegend & 405317 \\
\hline \multicolumn{4}{|c|}{ Astrocytic (GFAP) Antibodies } \\
\hline Primary $\mathrm{Ab}$ & Rabbit GFAP & Life Technologies & 180063 \\
\hline Secondary $\mathrm{Ab}$ & Goat anti-Rabbit FITC (Preadsorbed) & AbCam & AB97079 \\
\hline \multicolumn{4}{|c|}{ Vascular (VWF) Antibodies } \\
\hline Primary $\mathrm{Ab}$ & Sheep Von-Willebrand Factor & Cedar Lanes & CL20176A-R \\
\hline Secondary $\mathrm{Ab}$ & Donkey anti-Sheep TRITC (Preadsorbed) & AbCore & $\mathrm{AC} 15-0277$ \\
\hline
\end{tabular}

Table 2.2: Materials \& Sources for Experimental Protocol

Immunofluorescent staining began with antigen retrieval, where the brain tissue was heated in a citrate buffer (10 mM Citric Acid, 0.05\% Tween-20, pH 6.0). Heating was performed while brain slices were maintained at mild pressure between a glass slide and coverslip, held in place by the arms of a binder clip. This strategy was critical, as antigen retrieval without compression left the tissue slice fragile and wrinkled while this improved technique prevented the tissue from deteriorating. A detailed diagram of this technique and demonstration of its improved outcomes are shown in Figure 2.1. The temperature of the antigen retrieval solution was raised to 103-105 Celsius (above the typical literature standard of 100 Celsius) and maintained for 10 minutes, after which the tissue is left to cool for 20 minutes off of the hot plate. Antigen retrieval was unnecessary for the VWF and GFAP antibodies, but due to the Smi-311 antibody being composed of larger IgM molecules it was necessary to carry out antigen retrieval to maximize data quality. Antigen retrieval carries risks of destroying native epitopes, and hence should only be used when the benefits in staining outweigh the costs of potential tissue damage. 

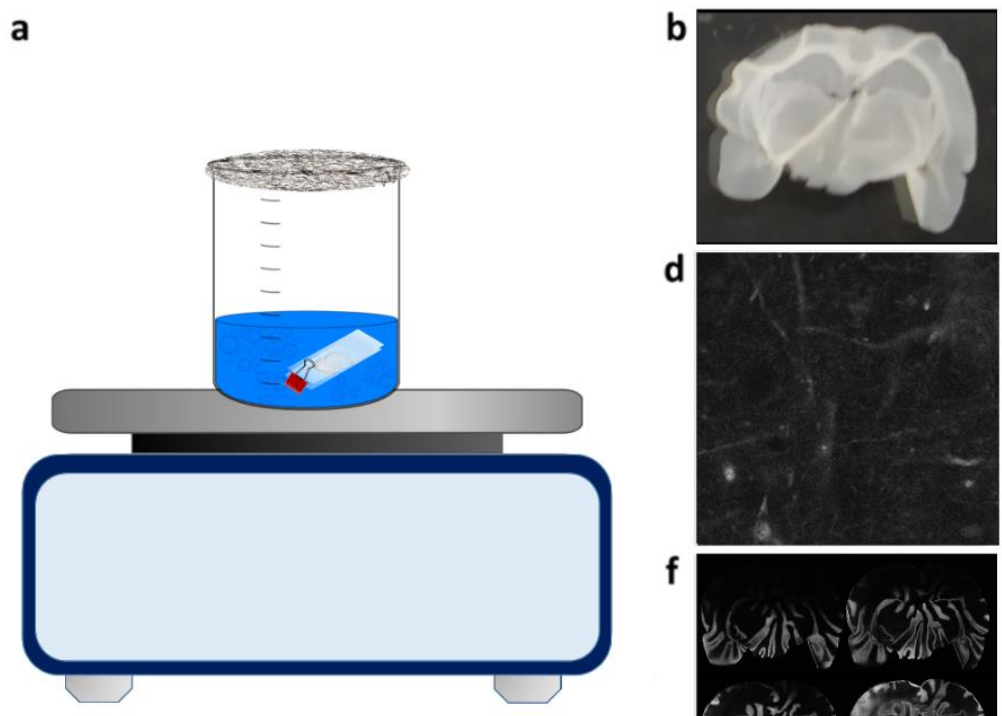

d

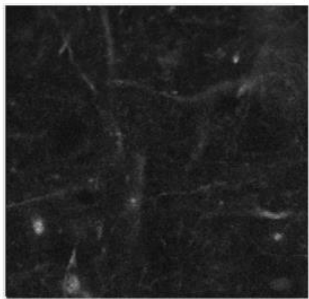

f

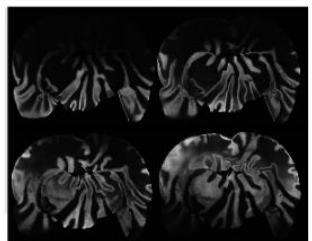

C

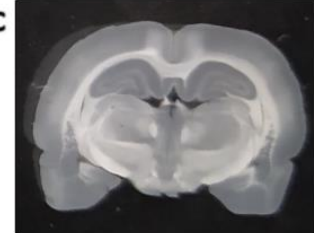

e
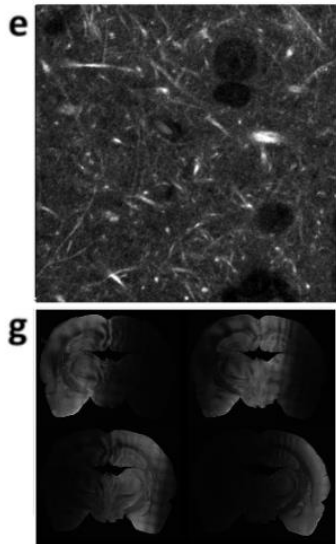

Figure 2.1: Antigen Retrieval Design \& Outcomes (a) A diagram of the antigen retrieval setup is shown. A brain slice is lightly compressed between two glass surfaces and boiled within the antigen retrieval solution. The arms of a binder clip are used to maintain the sandwich design. This setup has clear improvements in maintaining tissue health and minimizing wrinkling, as shown in the post-antigen retrieval slice images demonstrating $(b)$ antigen retrieval tissue appearance without compression and $(c)$ our improved strategy. Antigen retrieval was critical for improving Smi-311 staining quality. We demonstrate this by providing representative images of Smi-311 stained cortical slices using our immunofluorescent staining protocol $(d)$ without and $(e)$ with the additional antigen retrieval setup. Additionally, a 10x tiled mosaic of nuclei staining of four z-sections of the slices shown in (b) and $(c)$ are provided in $(f)$ and $(g)$, providing further evidence that our technique ensures flatter tissue sections which provide higher quality data cubes. While it may appear that the staining in $(f)$ and $(g)$ is 'patchy' the four Z-sections shown highlights that only partial regions of the tissue are present in a single optical section. The wrinkly structure of $(f)$ would not allow a cortical column to be easily collected, while for $(g)$ the profile of a cortical column would be flat along its length since the flatness changes over long distances instead of over short distances (as in $f$ ).

All steps following antigen retrieval were carried out at 37 degrees Celsius on a low-speed shaker with an incubation volume of $600 \mathrm{uL}$ per tissue section. An extended staining protocol was used, encompassing 12 hour blocking, (3x) 6 hour PBS washes and 48 hour antibody incubations, as described in Table 1.1. The staining procedure was carried within a 3-well removable silicone immunofluorescent chamber (Ibidi \#80381). This protocol is substantially extended from traditional IHC procedure, due to a combination of two factors. First, Smi-311 primary antibodies were composed of an $\operatorname{IgG/IgM~mixture~}$ which diffused slower into the tissue. Second, the thicker tissue sections require extended staining in order to optimize resultant image quality in the tissue center. Traditional IHC with thinner tissue sections typically requires 1 hour of blocking buffer, primary antibody and secondary antibody incubation at room temperature, along with short 3x 10 minute PBS washes (R\&D Systems, 2017) (Synaptic Systems, 2018). In many cases, 1 hour at room temperature is considered interchangeable with overnight at 4 Celsius. A 
common trend in the literature is an increase in these durations for thicker tissue sections. One example using 20 and 40 micron thick sections utilized primary antibody staining overnight at room temperature and secondary antibody staining for two hours at room temperature (Grabinski et al., 2015) while another using 50 micron thick tissue sections similarly used overnight room temperature primary antibody incubation (Vercelli et al., 2004).

Even thicker tissue staining has been shown to require even more extensive incubation times. One protocol using vibratome sections between 50 and 200 microns in thickness utilize primary and secondary antibody incubation times of "2 overnights" each at 4 Celsius (K. Meyer et al., 2017). Another protocol using tissue sections 500 microns thick utilized 3 days each of primary and secondary antibody incubation at 4 Celsius, along with 3 hour wash steps (Gonzalez-bellido, 2012). Finally, a protocol using $1 \mathrm{~mm}$ thick coronal brain sections utilized 24 hour each incubation steps for primary and secondary antibodies at 37 Celsius as well as a 24 hour washing step at 37 Celsius (Khoradmehr et al., 2019). These various examples demonstrate that thicker tissue can commonly require more extended staining steps, which guided the rationale to utilize extended staining and washing durations.

In order to demonstrate that the extended protocol is necessary to maximize data quality for Smi311 staining (as GFAP and VWF did not require this additional extension), an extensive factorial design was carried out based on three parameters, each with two levels. These included antigen retrieval \{used or not used $\}$, staining duration $\{$ traditional or extended $\}$ and PBS wash duration $\{$ traditional or extended $\}$. This generated a $2 \times 2 \times 2$ factorial design, demonstrated in Figure 2.2. The combination of 'Traditional PBS Washing' and 'Traditional Incubation Times' was removed from the factorial design after finding that 'Traditional Incubation Time' + 'Extended PBS Washing' was unable to provide any signal for Smi-311 staining in the central region of the tissue due to the short antibody incubation. Hence, we decided not to waste additional antibody on a condition we knew would be insufficient for allowing antibodies to diffuse to the center of the tissue (due to the traditional antibody incubation time). 


\begin{tabular}{|c|c|c|c|}
\hline \multicolumn{5}{|c|}{$\mathbf{2} \times \mathbf{2} \times \mathbf{2}$ Factorial Design } \\
$\begin{array}{c}\text { Condition } \\
\text { Label }\end{array}$ & $\begin{array}{c}\text { Antigen } \\
\text { Retrieval }\end{array}$ & $\begin{array}{c}\text { Staining } \\
\text { Duration }\end{array}$ & $\begin{array}{c}\text { PBS Wash } \\
\text { Duration }\end{array}$ \\
\hline N/A & $\mathbf{x}$ & Traditional & Traditional \\
\hline N/A & $\checkmark$ & Traditional & Traditional \\
\hline 1 & $\mathbf{x}$ & Traditional & Extended \\
\hline 2 & $\checkmark$ & Traditional & Extended \\
\hline 3 & $\mathbf{x}$ & Extended & Traditional \\
\hline 4 & $\checkmark$ & Extended & Traditional \\
\hline 5 & $\mathbf{x}$ & Extended & Extended \\
\hline 6 & $\checkmark$ & Extended & Extended \\
\hline
\end{tabular}

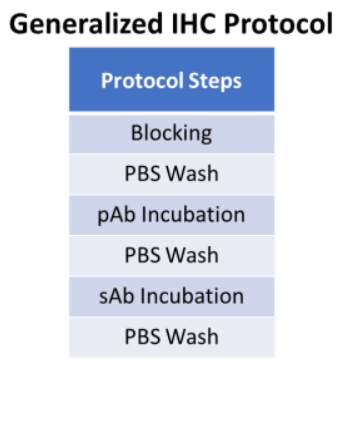

\begin{tabular}{|l|l|}
\multicolumn{2}{|c|}{ Factor Levels } \\
\hline \multicolumn{2}{|c|}{ Blocking } \\
\hline Traditional & $1 \mathrm{hr}, \mathrm{RT}$ \\
\hline Extended & $12 \mathrm{hr}, 37^{\circ} \mathrm{C}$ \\
\hline \multicolumn{2}{|c|}{ PBS Wash } \\
\hline Traditional & $(3 \mathrm{x}) 10 \mathrm{~min}, \mathrm{RT}$ \\
\hline Extended & $(3 \mathrm{x}) 6 \mathrm{hr}, 37^{\circ} \mathrm{C}$ \\
\hline \multicolumn{2}{|c|}{ pAb Incubation } \\
\hline Traditional & $1 \mathrm{hr}, \mathrm{RT}$ \\
\hline Extended & $48 \mathrm{hr}, 37^{\circ} \mathrm{C}$ \\
\hline \multicolumn{2}{|c|}{$\mathrm{sAb}$ Incubation } \\
\hline Traditional & $1 \mathrm{hr}, \mathrm{RT}$ \\
\hline Extended & $48 \mathrm{hr}, 37^{\circ} \mathrm{C}$ \\
\hline
\end{tabular}

Figure 2.2: Factorial Design for Smi-311 Staining Optimization. Demonstration of the $2 \times 2 \times 2$ factorial design for optimizing Smi311 staining, along with the generalized IHC protocol and the specific levels of each factor. The first two conditions were excluded ("N/A") from the study after finding that each sample of conditions ' 1 ' and ' 2 ' were unable to stain the center of the tissue section due to the short 'traditional' staining duration, indicating that the two excluded conditions would have had the same outcomes.

To carry out this study while ensuring valid comparison of the experimental conditions, the factorial study utilized a sequence of consecutive coronal brain sections that include the S1BF region. For each of the six conditions tested, three hemispheres were independently stained and imaged, providing 18 total hemispheric samples for this factorial study. The hemispheres were collected by cutting brain slices in half along their axis of symmetry. From each stained hemisphere, an entire cortical column of Smi-311 staining was collected in the S1BF region from the surface of the cortex to the grey/white interface. While the 'optimal' staining times for each step may be shorter than the extended times utilized, this was not further optimized within the study.

For the four-color staining experiments, tissue sections were carefully transferred out of 24-well plates following immunofluorescent staining and onto microscope slides using a fine-tipped paintbrush, after which they were mounted in glycerol, coverslipped and sealed with clear nail polish. In this study, an Olympus FV1000 and a Nikon C2+ confocal laser-scanning microscope was used with four separate nonoverlapping fluorescent channels to generate cortical column data cubes using 70 micron thick coronal tissue slices. These four channels are illuminated using a $405 \mathrm{~nm}, 488 \mathrm{~nm}, 561 \mathrm{~nm}$ and $640 \mathrm{~nm}$ laser. After placing the slide onto the microscope stage, the entire section is scanned at 10x in the nuclei channel in order to generate a high-resolution macroscopic image of the entire brain slice, as shown in Figure 2.3. 
(a)

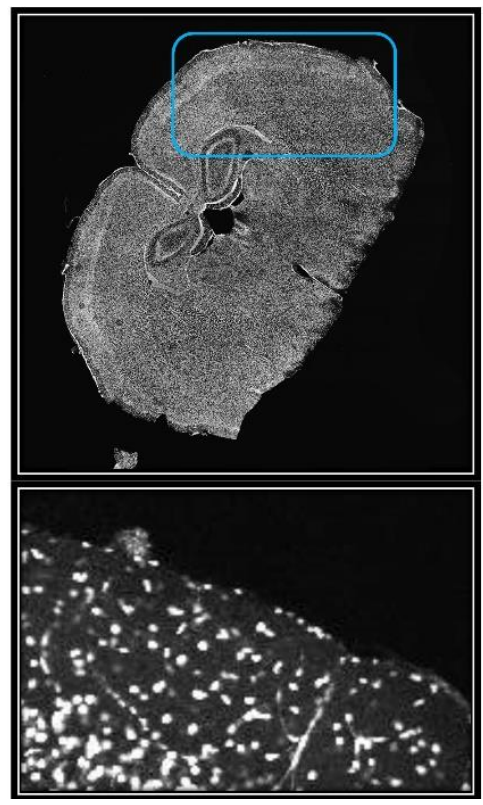

(b)

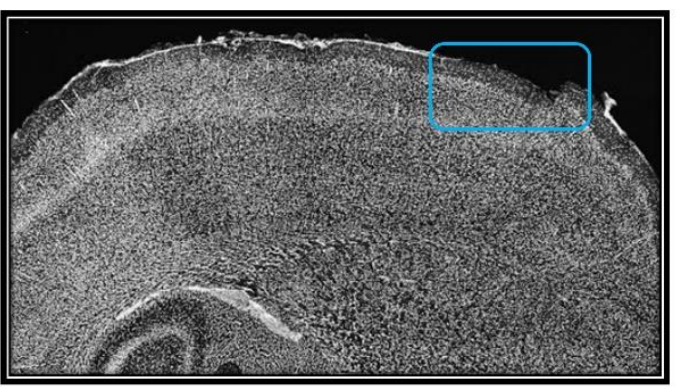

(c)

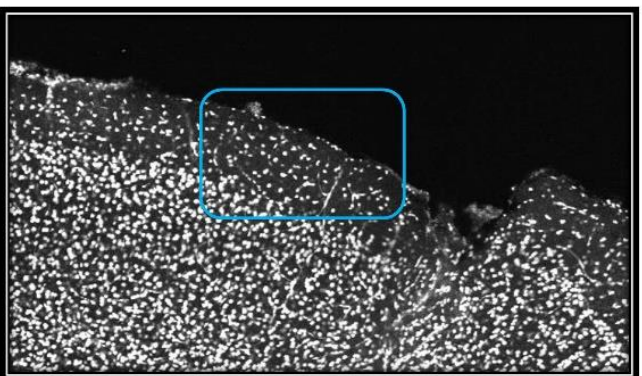

Figure 2.3: Demonstration of Nuclear Staining Mosaic. Representative image of nuclear staining of a rat brain slice composed of a stitched array of individual images collected at 10x magnification. Inset zooming from $(a)$ to $(d)$ demonstrates the extremely high resolution of the original image. This image also demonstrates the inherent difficulty of maintaining complete flatness of the brain slice on the slide due to the caution of avoiding excess compression of the sample. This is not a problem for the analysis of cortical columns, which is done on a smaller scale.

The corresponding image from the brain atlas was next matched to the immunofluorescently stained slice using landmark analysis, as discussed previously. This atlas image then underwent automated post-processing in order to highlight the various functional regions present in the particular brain slice.

Finally, the panel-scanned brain slice was then aligned with the post-processed brain atlas image, a strategy developed for this protocol and illustrated in Figure 2.4. This process allowed for a precise and quantitative relationship between image pixels and microscope stage coordinates (measured in microns), providing a convenient method to determine precisely which cortical region the microscope field of view encompasses even at high magnifications when it is far harder to determine where exactly in the slice the objective is viewing. Once at $60 \mathrm{x}$ magnification, it is critical to rely on stage coordinates since the field of view becomes too small too accurately determine the location within the slice being viewed. By aligning the two images, the stage coordinates corresponding to the 'four corners' of the cortical column were selected, which were then provided to the microscope companion software to automatically generate the individual stage coordinates of each image stack in the mosaic. 
(a)

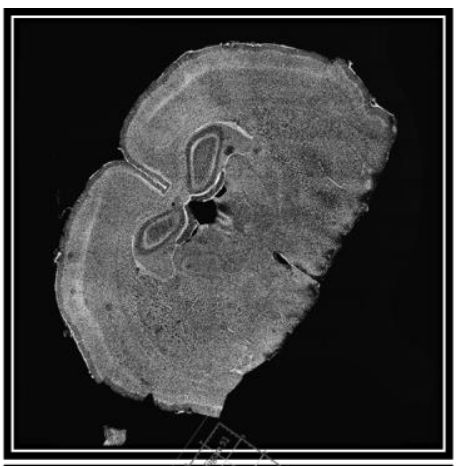

(c)

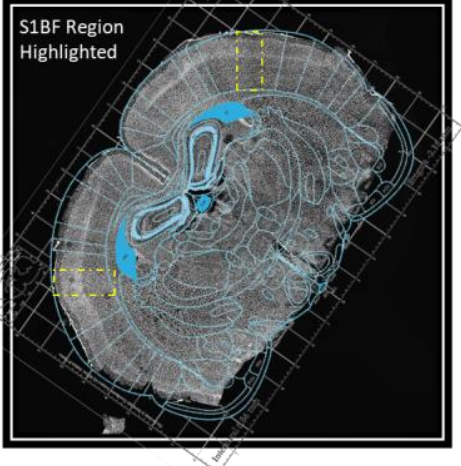

(b)

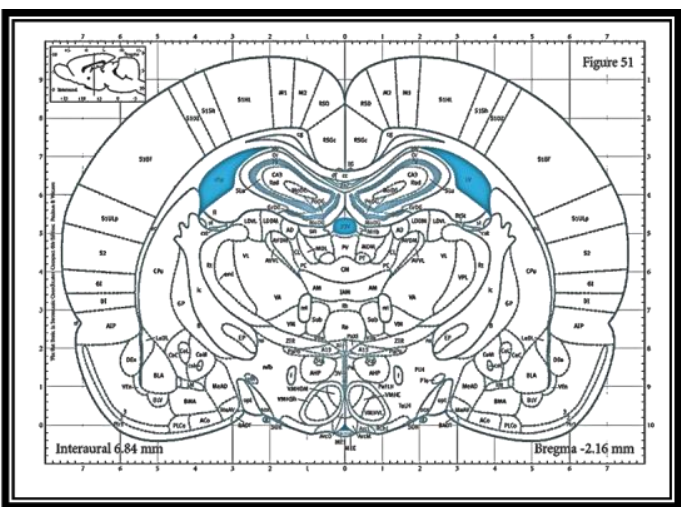

(d)

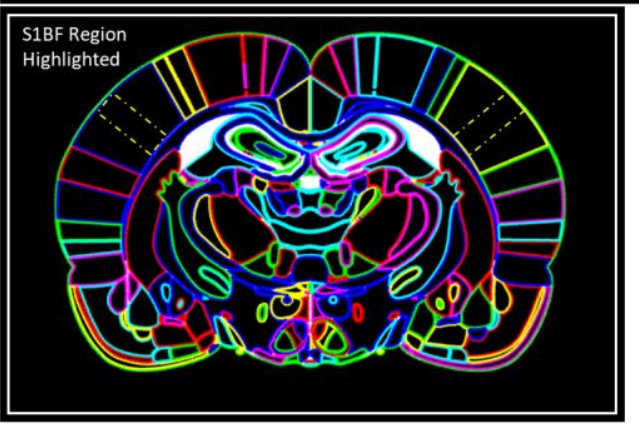

Figure 2.4: Cortical location localization algorithm. (a) First, the brain section is imaged through sequential imaging and stitching of individual 10x magnified images. (b) Next, landmark analysis of all sections collected through vibratome sectioning is used to locate the likely match from the Paxinos and Watson Atlas (Paxinos \& Watson, 2007). (c) A transparent overlay is used to align the two images, hence providing a convenient method of relating the microscope coordinates, image pixel locations from the stitched image and the functional region of the cortex. (d) An automated post-processing algorithm (leveraging the bwboundaries Matlab function) is also applied to the Paxinos and Watson atlas image to extract a pseudo-colored image that highlights all independent regions with random colors for better visual delineation. The approximate size and location of the S1BF cortical column is highlighted in $(c)$ and $(d)$.

Once the cortical region of interest was located, a multi-panel mosaic scan was conducted with a 60x objective of approximately $1 \mathrm{~mm}$ (cortical column width) by $3 \mathrm{~mm}$ (cortical column length) by 70 microns (thickness of slice). A full cortical column is typically five panels wide and sixteen panels in length. To capture the entire cortical column, typically $80 \mathrm{Z}$-sections are collected spaced 1- $\mu \mathrm{m}$ apart. Since separate raster scans of each panel are done for each of the four fluorescent channels, the number of individual scans is $5 \times 16 \times 80 \times 4=25,600$ scans per cortical column. A full scan typically requires approximately 100 Gigabytes of storage space. To manage the large datasets, we utilized an HP Workstation Z440 with an Intel Xeon E5-1650V4 CPU, 64 GB of ram and a Nvidia Quadro M5000 graphics card. This provided the desired volume of tissue to assess morphological connectivity in the cortical column, since within this large region was a dense interwoven network of cellular filaments with a maximum diameter of only 3-5 microns. Due to the small size of the constituent cellular filaments, there 
was more than enough volumetric space within each dataset to generate plentiful connectivity metrics for detailed statistical assessment.

There was no need to perform image correction for the cortical column orientation, as the slice was carefully mounted on the glass slide so that the desired functional region (M1 or S1BF) would lie along the stage axes. As the M1 columns were parallel on each hemisphere, the slice would be oriented so that its axis of symmetry was parallel to the vertical stage axis. Since the S1BF columns were perpendicular to one another, as they lie on the outer curvature of the coronal section, the slice is oriented so that its axis of symmetry is 45 degrees off-axis to the stage axes. This ensures that one hemisphere aligns with the horizontal stage axis while the other hemisphere aligns with the vertical stage axis. This can be clearly seen in the orientation of the S1BF cortical columns in Figure 2.3.

The entire scan typically took between 24 and 48 hours per cortical column, due to the fine spatial resolution (207 $\mathrm{nm}$ along the $\mathrm{XY}$ axis and 1 micron along the $\mathrm{Z}$ axis). This extended scan may be unrealistic for some labs but was the only way to capture such a large cortical column over the entire Zdepth of the cortical slice for all four fluorescent channels. This scan proceeds panel-by-panel across the entire mosaic, preventing constant illumination of the entire cortical column during the scan duration. In order to minimize photobleaching, our imaging strategy began with the most red-shifted fluorophores in the $640 \mathrm{~nm}$ channel and concluded with the most blue-shifted fluorophores in the $405 \mathrm{~nm}$ channel, since the UV illumination laser had a higher propensity for photobleaching than the $488 \mathrm{~nm}, 561 \mathrm{~nm}$ or $640 \mathrm{~nm}$ lasers. Finally, our protocol utilized a high concentration of antibody to allow us to use lower laser powers for illumination and a faster pixel scan rate. The finished collection of images was then stitched together utilizing Terastitcher (Bria \& Iannello, 2012).

\section{Results}

As a result of the factorial design to optimize Smi-311 staining quality, each of the six experimental conditions yielded three complete cortical columns from three distinct hemispheric sections. In order to objectively quantify the image quality to carry out statistical comparison of each condition, a published methodology by Koho et al (Koho et al., 2016) was utilized. This methodology works through 
characterization of the spatial entropy and power spectrum of the image in order to define 'image quality' as an image with high contrast - which corresponds to brighter filaments against a darker background. This is believed to be a valid method of comparison as all cortical columns were imaged using the same microscope imaging parameters. The author of this methodology released their code as an open-source Python package, which was utilized. The package quantifies the quality of each image without the need of any user input, ensuring the final result is completely objective. In order to maximize the validity of comparisons from the factorial designs, all cortical columns were collected from consecutive slices from a single brain. The use of consecutive slices minimizes intrinsic variability between slices but due to this all results from the factorial study only emerge from analysis of a single rat brain. From each cortical column, a $207 \times 207 \times 70 \mu \mathrm{m}$ data cube [1000 x $1000 \times 70$ pixels] was extracted encompassing the entire tissue thickness (70 $\mu \mathrm{m}$ using $1 \mu \mathrm{m} \mathrm{Z}$-sections) at two specific depths into the cortical columns $-600 \mu \mathrm{m}$ and $1200 \mu \mathrm{m}$. At each of these cortical depths, within the data cube, Z-sections were extracted at five specific Z-positions $\{5 \mu \mathrm{m}, 20 \mu \mathrm{m}, 35 \mu \mathrm{m}, 50 \mu \mathrm{m}$ and $65 \mu \mathrm{m}\}$. These five $1000 \times 1000$ pixel images in each of the three cortical columns within each of the six conditions were used with the python package to objectively quantify the image quality. In Figures 2.5 and 2.6 outcomes are provided at these two cortical depth conditions. At both the $600 \mu \mathrm{m}$ and $1200 \mu \mathrm{m}$ cortical depth condition, it is clear that the developed protocol, combining antigen retrieval, extended Smi-311 staining and extended PBS washing provides statistically significant improvements in image quality over the other five conditions at the 'center' of the tissue (35 $\mu \mathrm{m} \mathrm{Z}$-section), although these effects become insignificant when approaching tissue edges. Furthermore, it is evident that 'Traditional Staining' does not provide enough diffusion time for the antibodies to reach the center of the tissue. It is not entirely clear the mechanism by which the extended washing provides improved image quality, but more critical analysis of this phenomena can be left to future studies. 
(b)

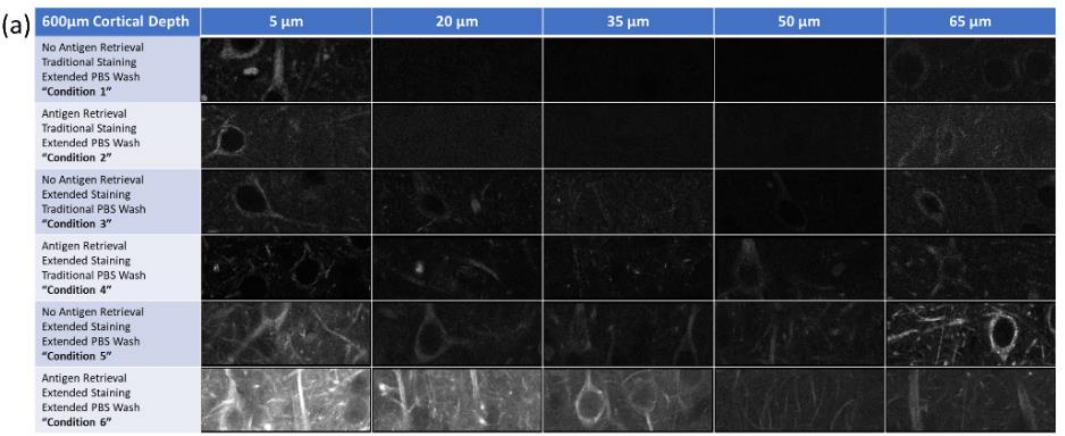

(c)

Image Quality by Depth \& Condition - $600 \mu \mathrm{m}$ Cortical Depth

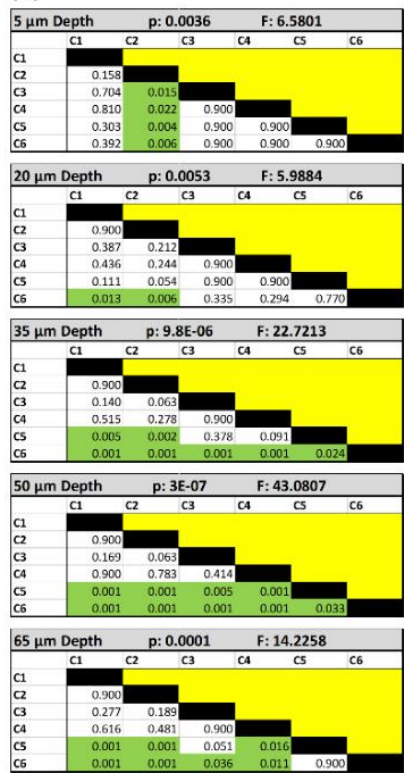

Figure 2.5: Factorial experiment results at $600 \mu \mathrm{m}$ cortical depth. (a) provides a representative image of the staining quality of each condition at each Z-depth. (c) provides the average and standard error bars of the image quality metric of each condition at each measured Z-depth. Standard error bars are computed using standard error of the mean (SEM) and reflect the variability from three independently collected samples per condition per depth. (b) provides the ANOVA and post-hoc Tukey testing used to compare each of the six conditions at each Z-depth. The first line of each ANOVA provides the overall $p$ and $F$ value, which in each case demonstrate a statistically significant difference between the conditions. The grid below this line compares each condition through post-hoc Tukey testing, with green highlighting for statistically significant differences.

(b)

(a)

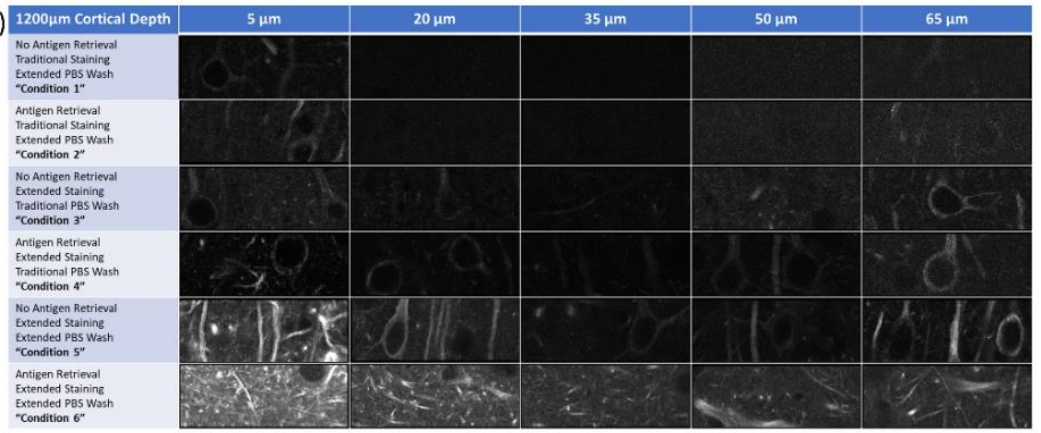

(c)

Image Quality by Depth \& Condition - $1200 \mu \mathrm{m}$ Cortical Dept
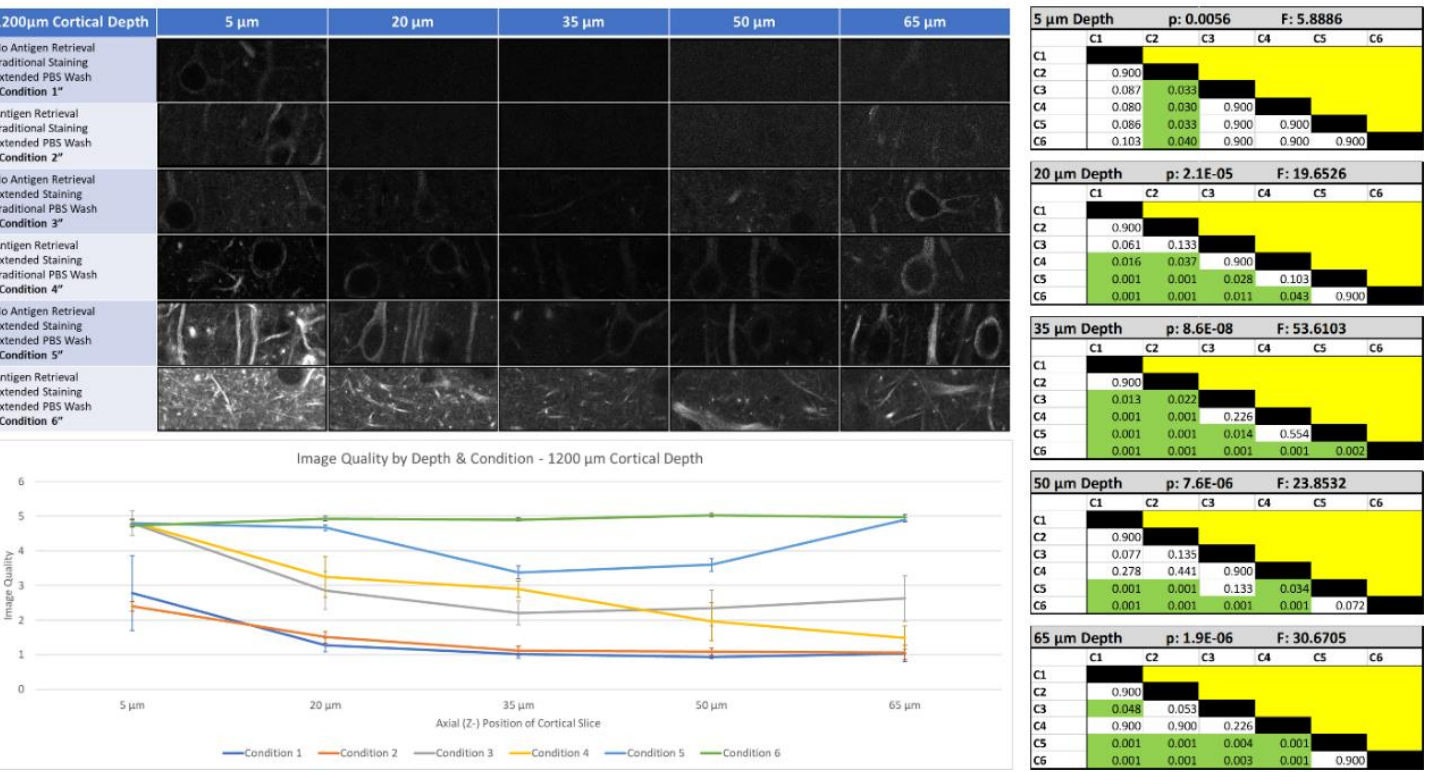

Figure 2.6: Factorial experiment results at $1200 \mu \mathrm{m}$ cortical depth. (a) provides a representative image of the staining quality of each condition at each Z-depth. Standard error bars are computed using standard error of the mean (SEM) and reflect the variability from three independently collected samples per condition per depth. (c) provides the average and standard error bars of the Image Quality metric of each condition at each measured Z-depth. (b) provides the ANOVA and post-hoc Tukey testing used to compare each of the six conditions at each Z-depth. The first line of each ANOVA provides the overall $p$ and $F$ value, which in each case demonstrate a statistically significant difference between the conditions. The grid below this line compares each condition through post-hoc Tukey testing, with green highlighting for statistically significant differences. 
Regarding the four-color staining and imaging scheme, various features of cellular interconnectivity throughout the entire tissue slice thickness can be visualized in figure 2.7 , including astrocytic cuffing of capillaries and nuclei localization inside the unstained soma of neurons.
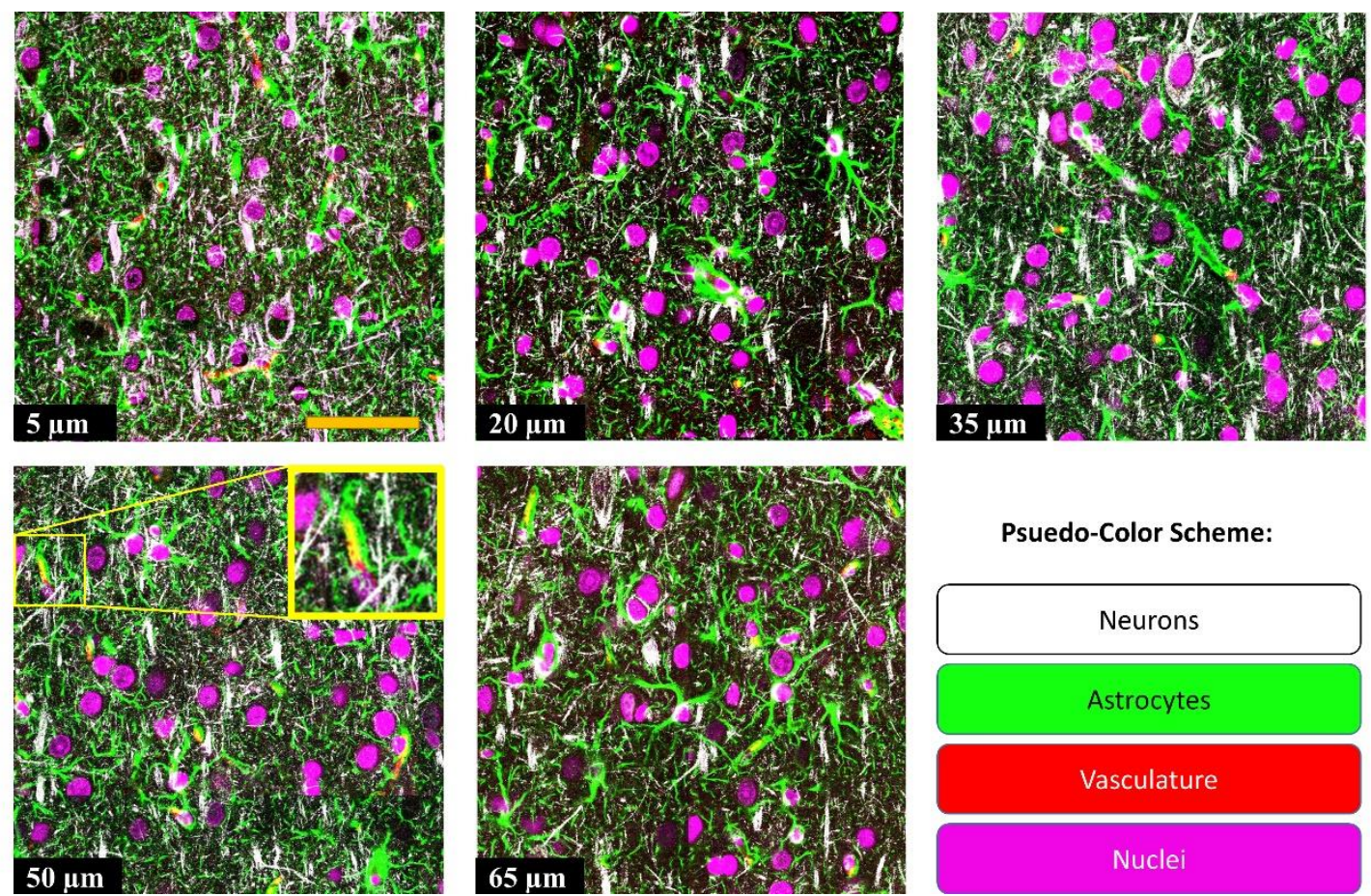

Psuedo-Color Scheme:

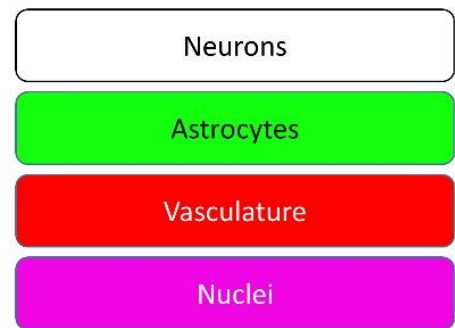

Figure 2.7: Four-color staining and cellular architecture at various depths. Inset figure at 50 um depth demonstrates the presence of stained components around an astrocyte-cuffed capillary. The yellow color on vascular surface highlights the proximity of a stained astrocyte and the capillary it is cuffing. The scale bar at 5 um depth represents a distance of $50 \mathrm{um}$.

However, it is also essential to point out that the data subsets identified in the preceding visualization are only a small proportion of an entire cortical column. As demonstrated in figure 2.8 , the actual datasets are far larger than those from the preceding images. From this macroscopic perspective many of the finer details are no longer visible unless extensively magnified. 

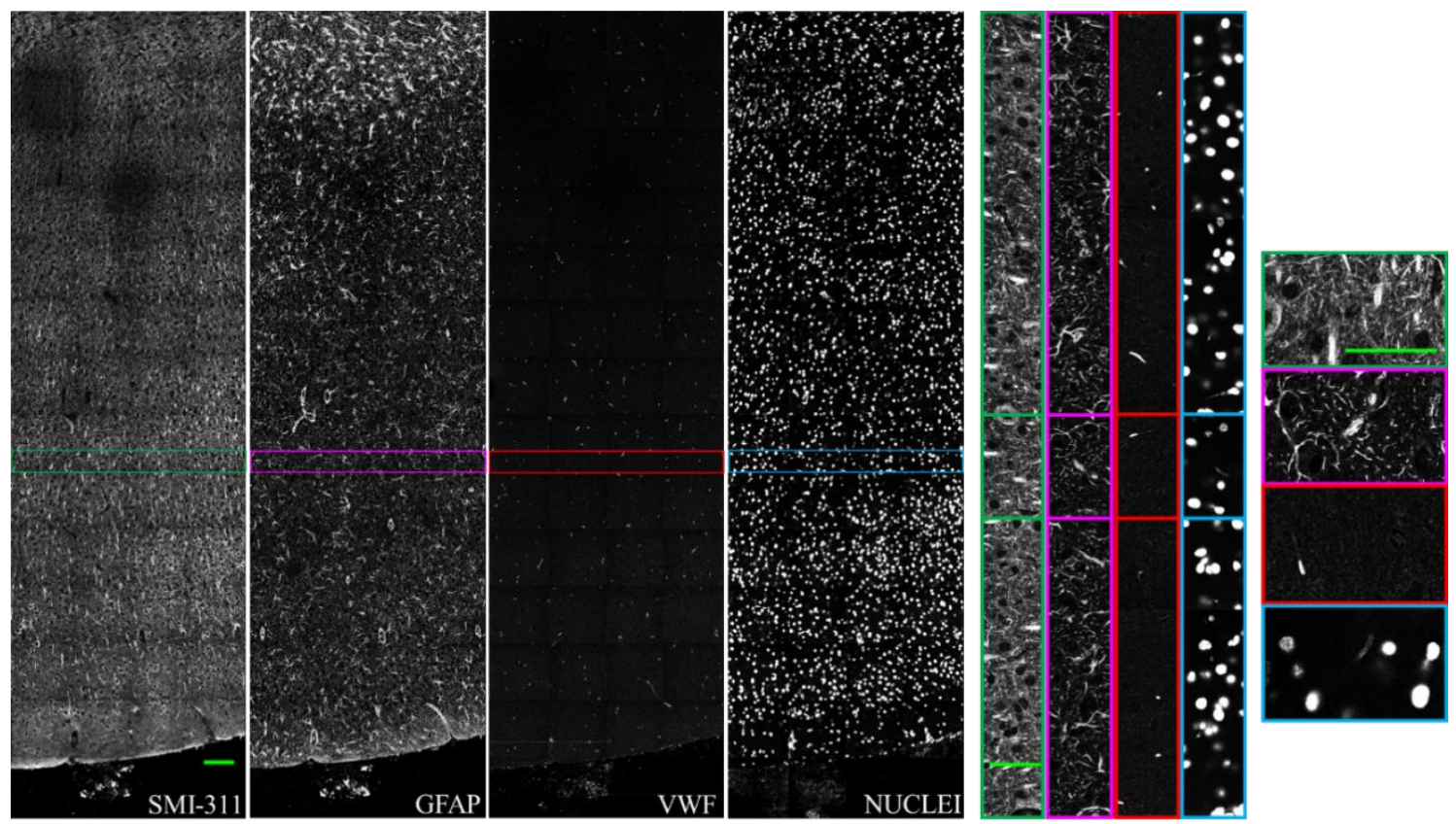

Figure 2.8: Size and scope of a single Z-section in a typical cortical column data cube. Color-coded inset boxes for each channel demonstrate two sequential magnifications to highlight the filament density and staining quality. Each successive magnification includes a 90 degree rotation for ease of visibility. The green scale bar in each set of images represents a distance of 50 microns.

One of the fundamental challenges which also arise is the decline in fluorescent intensity with imaging depth into the tissue, caused by a combination of photobleaching, optical absorption and antibody diffusion limitations. To resolve this, a built-in plugin was utilized within ImageJ called Stack Contrast Adjustment ("SCA"), which is designed to maintain the brightness of an image throughout an entire stack by adjusting the histogram of the image slightly on a per-frame basis (Čapek et al., 2006) (Schindelin et al., 2012) (Schneider et al., 2012). This step is essential to maintain the brightness of cellular filaments throughout the image stack, ensuring that later visualization methods will equally weigh deeper filaments as well as shallower filaments. An example of the outcomes of this technique is shown in Figure 2.9. 


\section{Raw Smi-311 Data}
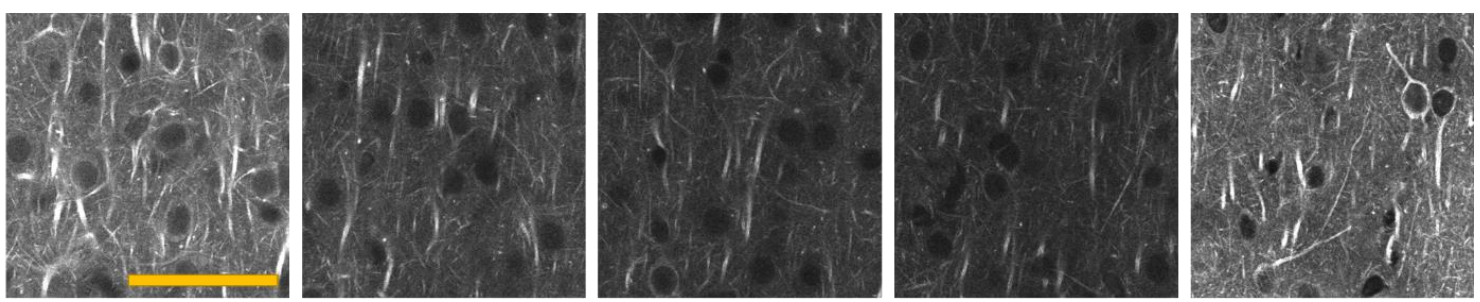

\section{$5 \mu \mathrm{m}$}

\section{$20 \mu \mathrm{m}$}

$35 \mu \mathrm{m}$

$50 \mu \mathrm{m}$

$65 \mu \mathrm{m}$
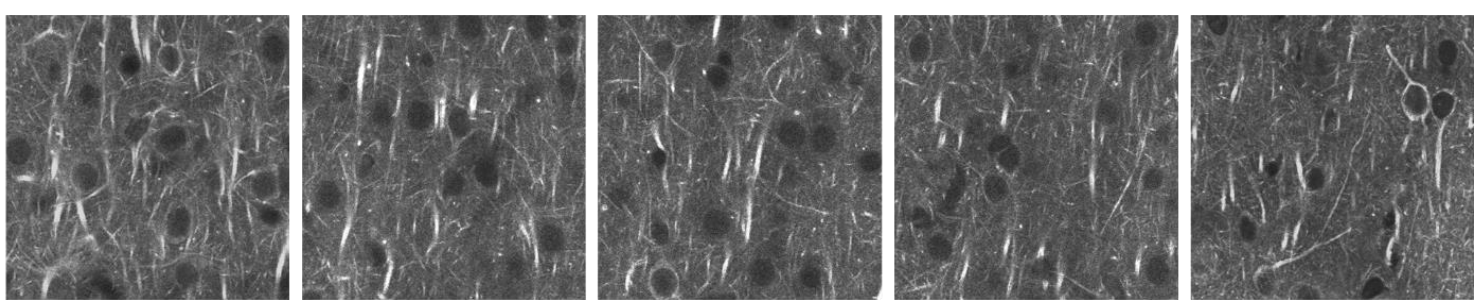

\section{Stack Contrast Adjusted Data}

Figure 2.9: Smi-311 Staining Enhancements Following Stack Contrast Adjustment (SCA). This figure highlights the improved stability of contrast throughout the tissue thickness. While the differences in image contrast at the tissue center may appear minimal, the standardization of contrast across the entire tissue thickness is critical for later volumetric modeling, which requires a consistent contrast level throughout the data cube. The yellow scale bar reflects a distance of 50 microns.

Once the image data has been corrected, the final data becomes suitable for further analysis. Due to the three-dimensional structure of the datasets, they lend themselves to a unique visualization opportunity. Utilizing Imaris Bitplane modeling software, a volumetric representation can be constructed of the assembled data to better visualize the network architecture of the cortical region. This is demonstrated in Figure 2.10. While Imaris is a valuable tool for data visualization and analysis for image stacks, other software that is available for researchers include Bioimage XD, FIJI, ImageSurfer, Osirix, SVI Huygens, Vaa3D and VisBio. 


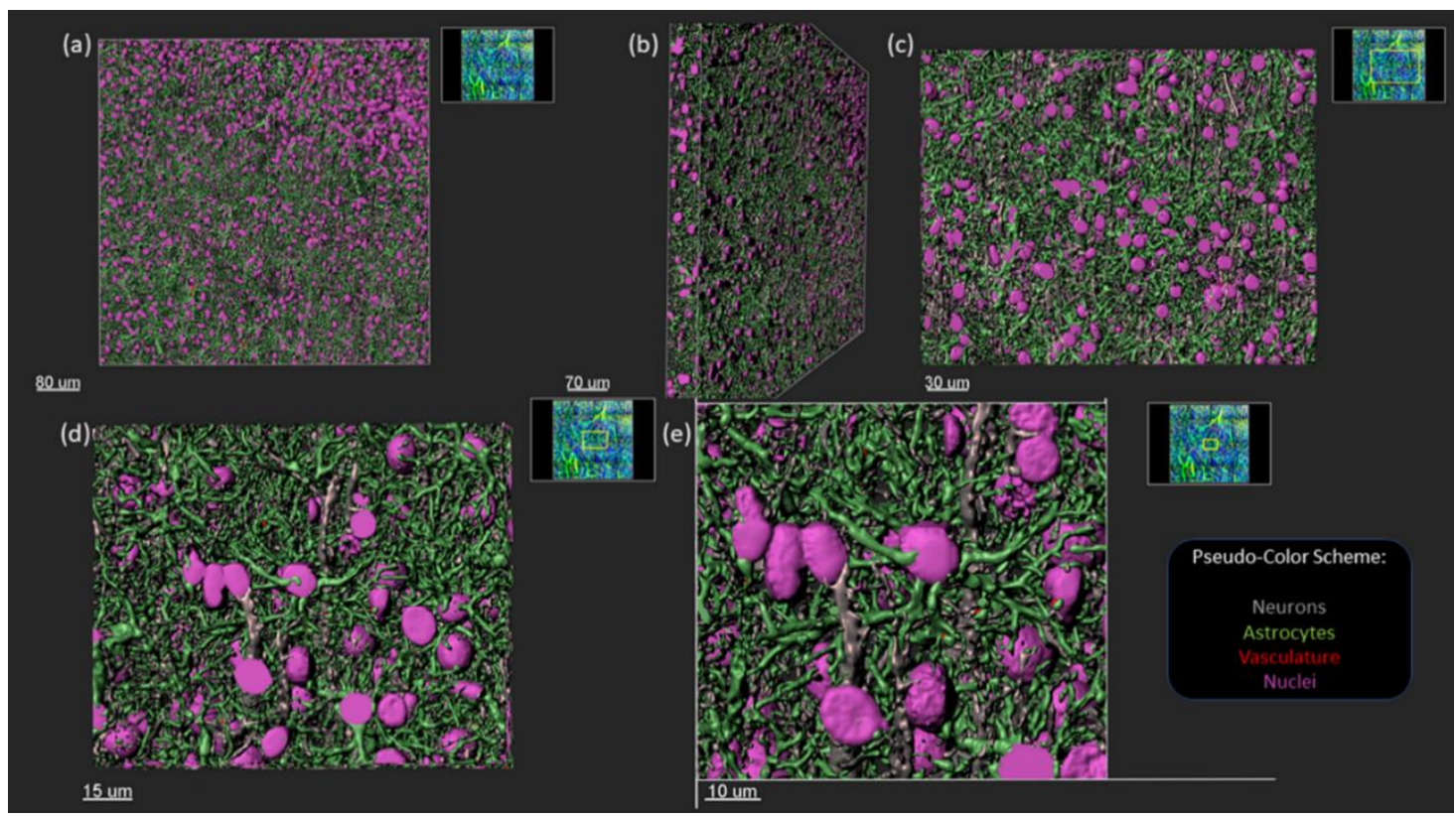

Figure 2.10: Volumetric Visualization of Cellular Interconnectivity. Demonstration of the interwoven nature of all cellular filaments in three-dimensions, aided by the data visualization properties of Bitplane Imaris Software. Sequential zooming and rotation highlight the dense filament connectivity in the neuropil. Data has not undergone deconvolution, and hence exhibits axial stretching due to intrinsic PSF-driven blurring.

\section{Discussion}

This methodology provides substantial improvements over existing techniques for both immunofluorescent staining and subsequent imaging. Compressed antigen retrieval alongside a combination of immunofluorescent staining targets provides insight into network architecture of critical cortical components. Specific improvements of the extended immunofluorescent protocol are demonstrated objectively and quantitatively through a detailed factorial design. Utilization of a lowresolution 10x full-slice mosaic to perform section registration to the rat brain atlas followed by ROIidentification and high-resolution scanning of the entire cortical column provides improved localization of desired functional region of interest. These accomplishments demonstrate a variety of staining and wholeslice imaging strategies which aim to provide guidance for other research groups in two ways. First, it may provide insight towards multi-component staining in combination with large scale imaging and functionalregion identification. Second, it can assist in the development of factorial experiment design for quantifying and optimizing staining protocol outcomes. 
To maximize the homogeneity of antibody diffusion throughout the tissue slice, antigen retrieval played a critical role. Optimizing staining outcomes requires the full penetration of primary and secondary antibodies throughout the entirety of the tissue section. However, because of paraformaldehyde fixation, antibody-epitope binding is substantially reduced due to the formation of cross-linked methylene bridges (Scalia et al., 2017). These methylene bridges reduce both the diffusion rate of antibodies into the tissue as well as their binding efficiency. To restore binding efficiency, an antigen retrieval process is carried out and the tissue is boiled within a citric acid solution. While not all antibodies required this form of pretreatment, the process proved critical for Smi-311. This is attributed to the characteristics of the primary antibody, which was composed of a mixture of Immunoglobulin G ("IgG") and much larger Immunoglobulin M ("IgM") antibodies. The IgM antibodies naturally diffuse slower into tissue, and thus especially benefit from the antigen retrieval process. Furthermore, at the typical boiling temperature at atmospheric pressure, 100 degrees Celsius, the effect of antigen retrieval was found to be far less substantial than when boiled in a sealed beaker - which yielded an enhanced temperature of 103 Celsius. During earlier optimization, boiling was performed for 5, 10, 15 and 20 minutes. However, the tissue became too fragile for use when boiling at 15 or 20 minutes was used, so 10 minutes was chosen for the study. A separate study to identify the optimal interaction of temperature and time would prove valuable to further uncover the optimal conditions. Furthermore, while quantitative assessment of image quality in the factorial design identified additional wash steps as enhancing image quality, a clear understanding of the underlying mechanism would be beneficial and requires additional study.

To reduce the risk of damage to the tissue section during antigen retrieval, the brain section was entrapped between glass surfaces. While sample processing should leave the mechanical properties of the tissue slice unaltered, the antigen retrieval process carries substantial risks to tissue health. Bubbles formed during the boiling process can tear thin sections of tissue due to repeated agitation of sample. Furthermore, the combination of high temperatures and long heating durations can substantially wrinkle the tissue section and reduce its mechanical strength to the point where it will easily break into smaller pieces during transfer. Even when the degree of tissue wrinkling appears minor, wrinkling prevents optimal sample mounting within the imaging chamber and severely distorts the final 3D-modeled data cube. Additionally, wrinkles often increase the overall thickness of the sample beyond the focal length of the objective, leading 
to compression of the tissue slice by the objective when imaging the deepest regions of the thick tissue slice. As a result of the compression of these wrinkles during scanning, the final stitched data becomes discontinuous and can suffer from tearing artifacts. Measurement of small areas will likely be unaffected by these challenges, but for large area scanning, such as in this study, maintaining the integrity of cortical tissue is critical. In this study, the risk of these artifacts is substantially reduced by carrying out antigen retrieval while the brain slice is uniformly and lightly compressed between a glass slide and coverslip. Application of this pressure during the antigen retrieval process protects the tissue slice from both bubbles and warping/wrinkling.

The four-color protocol provided has advantages as well as tradeoffs. Simultaneous staining of neurons, astrocytes, vasculature and nuclei provides an important opportunity to examine cortical network architecture, although labelling limitations emerge with so many independently stained components. Despite the focus of staining structural instead of functional markers, a deeper understanding of the underlying 'hardware' of the cortex can provide insights as to the nature of the differences in cellular communication (Samborska et al., 2016), both in a depth-dependent and region-dependent manner. Each of these staining targets, while well-established for morphological identification, carries potential limitations. GFAP is only a staining agent for GFAP+ astrocytic processes, which although being a commonly used and accepted marker for astrocytes fails to bind to the finest peripheral astrocytic processes, which typically fall between 50-200 nm in diameter (Haseleu et al., 2013) (Lavialle et al., 2011). However, filaments this small already fall below the diffraction limit for typical optical microscopy techniques. Individual-cell microinjection can stain these finer filaments (Ogata \& Kosaka, 2002), but this technique is too time-intensive (and hence nearly impossible) for large-scale analysis. Furthermore, the Smi-311 stain is only for cell bodies and dendrites of neurons, hence preventing any analysis of axons or terminal regions. Finally, the staining of vasculature is carried out using an endothelial cell marker, VWF, which does not provide any information on the outer layers of larger vessels or any indication on whether detected vasculature is an artery or vein. However, for the purposes of this study this distinction is not necessary. RECA-1 would likely serve as a more suitable staining target but was unavailable for use at the time of study due to the unavailability of a suitable antibody host species that would not interfere with other antibody pairings. In order to maximize antibody penetration into the center of the tissue, 48 -hour antibody 
incubation steps were used, causing the entire staining protocol to take two weeks. This was especially important for the large Immunoglobulin M antibodies utilized. To further improve the existing staining protocol, it may be useful to utilize pre-conjugated primary antibodies to reduce the overall length of the staining protocol since primary and secondary antibodies are applied simultaneously. Additionally, antibody fragments can be used in place of the whole immunoglobulin antibodies when available to enhance diffusion rates into the tissue (Deonarain et al., 2018) and minimize the duration of incubation required.

One of the fundamental challenges faced when working at high magnifications within tissue slices is remaining confident in the location of the specific region being assessed. To resolve this problem, landmark analysis of the tissue sections was used in conjunction with a stereotaxic rat brain atlas to estimate the specific locations of functional regions of interest in each brain slice. While this has the advantage of more accurately estimating the location of specific functional regions, vibratome slicing of the brain that is not perfectly coronal due to imperfect mounting on the vibratome stage can reduce the accuracy of the stereotaxic assessment. To reduce the chance of imaging the wrong functional region, cortical columns were selected from the center of the regions of interest.

The use of 70 micron thick tissue sections is a limitation caused by the effects of optical absorption and scattering as well as the working distance of the confocal microscope objective. This thickness limitation prevented full neuronal reconstruction. Furthermore, due to uncertainty in in the vibratome sectioning angle relative to the normal vector from the brain surface, major neuronal arbors often appear at a sharp angle relative to the plane of sectioning, reducing the distance that a single neuron can be traced. This problem can be minimized by using a thicker tissue block, high focal-length, highmagnification objectives, tissue clearing (to minimize absorption-based degradation in image quality with depth) and axial PSF minimization (such as through multiphoton microscopy). Another potential solution to this problem is to extract cortical sheets through flattening of the cortex, which has been performed in previous studies (Lauer et al., 2018). However, while this will standardize the angle of imaging relative to the cortical surface, experimenters will be limited by the observation distance into the brain - a challenge that is not present when using the coronal sections within this study. 
Fluorescence microscopy with existing constraints on simultaneous labeling cannot typically achieve more than four channels in parallel. Accordingly, this methodology fails to identify other cell types of importance within the region, such as microglia, oligodendrocytes, pericytes or schwann cells, or any of the plethora of functional markers that accompany typical neuroscience studies. The fundamental restriction that prevents this is the limit of four simultaneous fluorescent channels that most research microscopes face. To overcome this limitation and add additional fluorescent channels, spectral acquisition and unmixing techniques will be necessary to handle the expected spectral overlaps (Valm et al., 2016), which in turn will significantly reduce acquisition speed due to the need to compute spectral data for each voxel.

In order to capture the full dendritic architecture of a neuron, it may be necessary to instead pursue alternative imaging technologies, such as multiphoton or light sheet microscopy. Multi-photon methods have distinct advantages, such as a smaller point-spread function, improved penetration depth and less photobleaching (Tauer, 2002). This improved penetration depth if used in parallel with a longer workingdistance objective would allow thicker sample imaging, which would increase our chances of entire neural dendrite reconstruction. Light sheet microscopy similarly provides enhanced imaging volume, more rapid imaging speeds and an optimized point-spread function due to decoupling of illumination and excitation pathways (Santi, 2011). Both technologies provide critical advantages, but at this time they have still not been optimized for simultaneous assessment of four separate markers. Multi-channel imaging with multiphoton microscopy has at most been able to assess three simultaneously stained markers using two unique excitation wavelengths, which when synchronized can undergo a non-linear sum-frequency generation process to provide a third excitation energy level (Mahou et al., 2012). The same author has been able to extend these techniques to light sheet microscopy as well through a mixed multiphoton light sheet microscope, which was similarly capable of three-channel collection (Mahou et al., 2014). While confocal techniques are typically inferior based on resolution and depth penetration, they are still the most available method for four-color simultaneous staining and imaging.

The outcomes of this immunofluorescent staining and imaging protocol demonstrate the capability of identifying network architecture of neurons, astrocytes and vascular processes simultaneously within cortical brain tissue. Through future volumetric and filamentous modeling, this extensive image data will 
be used to extract quantitative morphological metrics for analysis of neurovascular coupling, neurometabolic coupling and network architecture. This methodology holds promise for generating complex and accurate quantitative parameters to categorize the morphology of these cells and how they vary in different functional brain regions. For example, the heterogeneous nature of astrocyte form and function in the brain has been well documented in the scientific literature (Oberheim et al., 2012), but no comprehensive assessment of 3-dimensional morphology has been conducted.

Furthermore, if this methodology is utilized to assess diseased tissue it will be possible to obtain a more comprehensive understanding of how disease states alter network architecture. The totality of all data can be used in collaboration with computational neuroscientists to utilize actual experimental data as inputs for existing computational models of brain complexity, as has been previously performed in the Virtual Brain Project (Ritter et al., 2013). The various applications of these comprehensive datasets are still being explored but hold substantial potential for better understanding patterns of connectivity in motor and somatosensory cortical zones. Ultimately, the largest strength of this methodology is the unique combination of staining targets, volume of investigation, spatial resolution and antigen retrieval strategy. In combination, these components provide an intriguing capability for enhanced cortical architecture analysis.

It is necessary to clearly discuss several key weaknesses of this methodology. First, the various optimizations performed in this study were developed for the specific collection of antibodies utilized and is thus not as directly applicable in different contexts. The focus in developing this methodology was not to create a universally generalizable protocol but instead to reflect on solutions to the various challenges faced. The challenges faced in this protocol primarily stemmed from two sources. First, the antibody pairing used for Smi-311 created a variety of issues (i.e. necessity of enhanced antigen retrieval temperature and extended staining protocol) in achieving optimal staining. While other antibody targets can be used for a similar purpose, or potentially even a combination of a separate neuronal nuclear stain and neurofilament stain, the staining quality achieved following optimization was sufficient to not warrant additional investigation of other staining markers, despite the extensive optimization which was required. The second challenge faced was due to the use of the VWF vascular antibody pairing. Earlier experiments utilizing RECA-1 as a staining target provided far better staining quality, but due to conflicts between 
animal sources from antibody pairings, a different staining target was required. VWF does a sufficient job of identifying vascular components, but is undoubtedly a lower-quality target due to not being expressed as ubiquitously in the vascular endothelium. Due to this, vasculature is indeed more difficult to identify within some of the visualizations provided that highlight the density of the neuropil. However, in many circumstances the sheer quantity of volumetrically modeled GFAP and Smi-311 filaments make visualization of any vasculature impossible since these filaments are too dense to allow examination beyond the most superficial layer. While this may reduce the visibility of the vascular staining within the volumetric visualizations due to its relatively lower density, it still exists and can be useful for later quantitative analysis.

An additional limitation emerged from the imaging process. Due to the sheer density of neurofilaments, there was additional background in the Smi-311 channel due to point-spread function blurring from fluorophores directly above and below the optical section shown. While confocal microscopy reduces this issue substantially as compared to widefield microscopy, intrinsic spherical aberration in the image formation process makes this an unavoidable issue.

Despite the challenges from these antibody pairings, the achieved contrast was sufficient to complete this four-color staining methodology in preparation for upcoming volumetric modeling and analysis. Utilization of alternative staining markers could have similarly prevented the need for sequential staining and substantially reduced the immunofluorescent staining protocol duration. However, once achieving satisfactory staining quality the pilot study was completed before attempting additional optimization. 


\section{Chapter 3. Depth-Variant Deconvolution \& Fractional Volume Analysis}

\section{Introduction}

A clear understanding of the variation of cell density in a depth-dependent and functional regiondependent manner in the healthy brain can allow for the development of a quantitative baseline to better map the progression of various disease states. Within the dense neuropil of the cerebral cortex, however, this is a tremendous challenge since the density of major cellular components cannot be accurately characterized as their highly interconnected nature makes accurate determination of individual cells nearly impossible. While dye-filling individual cells can provide cell individualization, it is extremely timeconsuming and unsuitable for analyzing cell density over large regions or many individual cells (Lai et al., 2017). Since cell individualization for calculation of absolute cell density is fundamentally problematic in the dense neuropil, fractional volume analysis (FVA) is an alternative approach to extract locationdependent cell density information. If individual cell volume does not change significantly through the cortical column, FVA can serve as a convenient index for depth or region-dependent cell distribution characteristics.

FVA has many benefits for researchers carrying out computational studies of cellular dynamics in the cerebral cortex. For example, understanding volume fractions of Astrocytes in a local region has been identified as a key factor in understanding glutamate uptake in the area (Medvedev et al., 2014) (Lehre \& Rusakov, 2002). Additionally, volume fraction measurements of dendritic spines has been incorporated into reaction-diffusion models for studying cAMP oscillations (Ohadi \& Rangamani, 2019). Similarly,

GABA concentration in the neocortex has been characterized based on the fractional volume of GABAergic neurons (Petroff et al., 2002). As additional computational strategies leveraging fractional volume of these cellular components emerge, they will be able to utilize this data to better understanding cellular dynamics in these regions.

FVA is also playing an increasing role in characterization of the quantitative metrics which emerge from new MRI techniques. One group (Wen et al., 2018) compared multimodality MRI data with gene expression data and uncovered a strong correlation between the R2t* metric of qGRE signals and the cellular composition of the human cortex, especially the volume fraction of neurons and glia. These results 
provide a potential means to assess microstructural changes in the in vivo brain during, for example, disease development. Similarly, another group has been able to link the quantitative outputs (fractional anisotropy, apparent diffusion coefficient and kurtosis) of diffusion MRI to local volume fractions of neuronal and glial components (Gilani et al., 2019) with the ultimate aim of using these relationships to monitor progression of diseases such as Alzheimer's and multiple sclerosis. These examples reveal the growing role of fractional volume analysis in better interpreting voxel-level quantitative metrics from noninvasive imaging modalities for the improved tracking of disease state progression.

Characterization of disease states is another activity where FVA is playing a useful role. For example, histological samples from patients with medial temporal lobe epilepsy undergo FVA of astrocyte processes in order to characterize the tissue samples as mild, moderate or severe astrogliosis (Witcher et al., 2009). Due to the well-established reactive Astrocyte swelling that occurs during astrogliosis (Robel et al., 2015), it is expected that FVA can serve as a useful tool for characterizing severity. Recent work has also identified FVA as a potentially critical tool for characterizing the stages of Alzheimer's disease progression (Domínguez-Álvaro et al., 2018). This study identified significant changes in the volume fractions of neuronal, glial and vascular components of brain sections, representing a potential future linkage with the MRI signal interpretation discussed previously in order to noninvasively characterize these parameters.

While FVA may not provide the individual-cell morphological metrics that alternative techniques can reveal, for characterizing regional vasculature FVA is one of the only tools available due to the inability to separate vasculature into discrete 'units'. Recent efforts to create a cerebral vascular atlas of the whole mouse brain utilized FVA to distinguish vascular characteristics of unique functional regions (Xiong et al., 2017). Assessments of the effects of ischemia on mice brain vasculature similarly uses fractional volume to characterize the resultant loss in vascular density (L.-Y. Zhang et al., 2018). Similar to this work, fractional vascular volume has also been characterized on a cortical depth-dependent basis from agranular, partly granular and granular cortex (Tsai et al., 2009). When quantifying vascular staining, FVA is one of the most optimal tools for characterizing volumetric properties.

In order to optimize the validity of the extracted FVA metrics, it is critical that the collected images represent the native tissue characteristics as closely as possible. The point-spread function (PSF) of the confocal fluorescent microscope is one of the most useful tools to characterize the potential aberrations 
in the collected data and represents the three-dimensional profile that would be captured by the imaging system resulting from a small fluorescent point source below the diffraction limit of light (Boutet de Monvel et al., 2001). Based on the understanding that a typical fluorescently stained object contains large quantities of these small point sources, the accuracy of volumetric measurements made on the resulting 3D image stack is limited by the extent of the blur created by a single fluorescent molecule. Complicating factors even further, the specific shape and size of the PSF is affected by the imaging wavelength, the microscope objective and the refractive index characteristics of the sample being imaged. Additionally, while scanning deeper into the sample, refractive index mismatches elongate the PSF with depth, a phenomenon known as spherical aberration, which further contributes to axial stretching of the data that worsens continuously the deeper into the sample being scanned. Fortunately, if the PSF can be wellcharacterized, deconvolution algorithms can be used to restore the collected imaging data to a condition closer to the original volumetric properties of the native tissue.

Numerous methodologies for deconvolution are available, all with the same fundamental objective of restoring blurred image data to a condition that better replicates the true characteristics of the tissue imaged - known as the 'ground truth.' One way to subdivide these methodologies is to consider three categories of PSF collection - (1) experimental collection of the microscope PSF, (2) generation of a theoretical PSF using imaging condition parameters and (3) estimation of the shape of the PSF using already imaged data. Experimental collection of PSFs are challenging due to the typically low SNR and need for repeated imaging and averaging, but provide the most accurate estimate of the blurring induced during the imaging process (Pankajakshan et al., 2009). Estimation of the theoretical PSF from experimental parameters (Aguet et al., 2008) (Ben Hadj et al., 2013) is a simple way to attempt deconvolution, but often provides less than ideal results due to substantial differences between the theoretical and experimental PSF. Recent methods for PSF estimation from imaged data utilize convolutional neural networks (Shajkofci \& Liebling, 2018) and generative adversarial networks (S. Lee et al., 2019), but without an a priori understanding of the imaging system aberrations these techniques are less than ideal. Another way deconvolution methodologies are subdivided are through consideration of blind vs non-blind methodologies. Blind methodologies attempt deconvolution by estimating the PSF from the raw collected image data, and then iteratively improving the estimate over multiple cycles of deconvolution. 
Non-blind methodologies rely on a fixed PSF, which remains unchanged over multiple deconvolution iterations.

Deconvolution strategies in the literature using experimental PSFs are often performed with only measurement of PSF from one 'depth condition.' However, some groups have identified a method where by immersing their fluorescent beads in agar gels they can identify PSFs at a range of depths to better characterize the precise degree to which spherical aberration impacts their image quality with depth (Boutet de Monvel et al., 2001). Another group has used measurements of a few select PSFs to interpolate PSFs at unique depths between the measured PSFs using fits of the PSF shape to Zernike moments (Maalouf et al., 2011).

In this study, we characterize FVA of neuronal, astrocytic, vascular and nuclear components in entire cortical columns of 70-micron thick coronal sections of murine brains, stretching from the cortical surface to the grey-white interface. FVA is calculated in 50-micron depth intervals along the $2.5 \mathrm{~mm}$ cortical column length for each component. Furthermore, we characterize FVA in two distinct functional regions of the cerebral cortex - the primary motor region (M1) and the primary somatosensory cortex barrel field (S1BF). To maximize statistical significance of the findings, eighteen of these cortical columns were collected over three different rat brains for each of these two functional regions. To optimize the validity of the metrics, an enhanced depth-dependent PSF collection and deconvolution scheme was provided which can apply independently to each fluorescent channel and throughout the depth of the thick tissue sections. From this data, both cortical depth-dependent and cortical depth-independent statistics are calculated in order to better understand whether these two vastly different functional regions have unique patterns of volume density of neuronal, astrocytic, vascular and nuclear components. Finally, individual nuclei density as well as estimates of astrocytic density are provided.

\section{Methods \& Materials}

Three adult male Wistar rats (16-20 weeks) were used to collect coronal brain slices for this study. Neuronal dendrites and soma were stained with Smi-311, astrocytes with GFAP, vasculature with VWF and nuclei with a staining kit (ThermoFisher R37113). Immunofluorescent staining and imaging 
techniques used have been described in Chapter 2. Briefly, antigen retrieval of lightly compressed slices were used to enhance antibody penetration of the Smi-311 primary antibody. An elongated staining protocol was utilized to maximize staining quality throughout the entirety of the tissue thickness.

Alignment between a 10x mosaic scan of the entire brain slice and a brain atlas was used to identify the location of the specific functional regions of interest. Within these functional regions, entire cortical columns were collected from the cortical surface to the grey/white interface through a mosaic scanning technique, encompassing data cubes $3 \mathrm{~mm}$ long, $800 \mu \mathrm{m}$ wide and the entire thickness of the tissue slice, $70 \mu \mathrm{m}$. Using a 60x oil-immersion objective, these large regions were scanned at a voxel size of $[0.207 \mu \mathrm{m}$ (x) $x 0.207 \mu \mathrm{m}(\mathrm{y}) \times 1 \mu \mathrm{m}(\mathrm{z})]$. From each of the three rats, three coronal slices were selected from the center of the M1 region and three from the S1BF region. From each of these slices, a cortical column was scanned from each hemisphere in the desired functional region. In total, this encompasses 36 total cortical columns, 18 from each of the two functional regions and 12 from each of the three rat brains.

Deconvolving data as accurately as possible requires recognizing that the PSF that blurs the ground truth data changes continuously while traveling from the tissue surface to the deepest $(70 \mu \mathrm{m})$ region of tissue. To measure the PSF, sub-micron fluorescent beads $175 \mathrm{~nm}$ in diameter (ThermoFisher \# P7220) were used to approximate a fluorescent point source, as they are below the resolution limit of a confocal fluorescence microscopy. Beads were acquired from all four fluorescent imaging channels used in the study in order to establish channel specific point spread functions. Estimating the PSF throughout this depth was accomplished through developing an imaging chamber to replicate the process of image formation through the brain slice. A diagram of this chamber is provided in Figure 3.1b. In short, the submicron fluorescent beads are first adsorbed onto the glass slide surface. A thin metal arbor shim disc with a hollow center is adhered to the slide, which creates a small well. The well is then filled with a refractive index matching liquid designed to mimic the refractive index of the grey matter of the cortex. $80 \%$ ethanol was chosen in order to most closely match the expected refractive index variability in the rat grey matter, which ranges from 1.36 to 1.37 (J. Sun et al., 2012). Finally, a coverslip is placed on top and the apparatus sealed with nail polish. We acquired four different thickness of arbor shims $-25 \mu \mathrm{m}, 50 \mu \mathrm{m}$ and $75 \mu \mathrm{m}$. These three conditions were tested in combination with a fourth condition where no arbor shim was used, replicating a condition at the surface of the tissue. By imaging the sub-micron beads through the RI- 
matching liquid at specific depths, it becomes possible to characterize the precise 3-dimensional point spread function of the confocal microscope used. At least 20 PSFs were collected from each of these four depth conditions, from each of the four fluorescent channels and from both laser-scanning confocal microscopes used in the study - a Nikon C2+ and an Olympus FV1000.

Within each cycle of imaging, numerous 3D PSF profiles are extracted. In order to extract a 'final' averaged PSF from the numerous PSF collected, the five highest quality PSFs collected were identified and then averaged. In order to rank the collected PSFs, two criteria were utilized - peak signal to noise ratio (PSNR) and photobleaching. In order to identify the PSNR, the maximum pixel value at the center of the PSF was divided by the average background noise level. If the brightest pixel value does not saturate the detector, a higher PSNR is desired as it provides the most substantial detail in the dimmer regions of the PSF. In order to characterize photobleaching, each region with beads underwent two successive rounds of Z-imaging. The ratio between the peak signal in the first and second round of imaging reveals the amount of photobleaching induced by the imaging process. Lesser photobleaching is ideal, as it reflects that the PSF is minimally altered during the Z-scan from which it is collected. From each combination of microscope, fluorescent channel and imaging chamber depth, the most ideal five PSFs were extracted based upon having the highest PSNR without saturation and the lowest photobleaching. All five beads were aligned along all three axes prior to averaging using the maximum intensity pixel.

Once averaging was complete, an optimal 3-dimensional PSF was identified for each condition. This is demonstrated in Figure 3.1a. In order to deconvolve the resultant data, the built-in blind deconvolution function within Matlab, deconvblind, was leveraged, which requires an initial guess of the PSF but iteratively improves its estimation over multiple cycles - hence falling in-between the typical 'blind' and 'non-blind' methodologies (Biggs \& Andrews, 1997) (Holmes et al., 1995). This has the advantage of utilizing experimental PSFs while also allowing for iterative optimization of their shape during the deconvolution process. Adjusting the PSF shape with each successive iteration is important considering the intrinsic heterogeneity of the PSF throughout the dataset (Jiang \& Wang, 2003). 10 iterations were chosen due to it being a standard cutoff point for microscopy deconvolution in the literature (Chen et al., 2013) (Biggs, 2010) and also due to experimental observations that additional iterations amplified background noise. Additionally, ten iterations was chosen Prior to deconvolution, a small 
$3 \times 3 \times 3$ median filter was applied to the dataset to preserve edges while reducing the presence of shot noise, which would introduce artifacts to the deconvolution process due to their high-frequency components $(\mathrm{Xu}$ et al., 2006). The PSF is filtered as well, as is typical in the literature (Hernández Candia \& GutiérrezMedina, 2014), in order to prevent any noise in the PSF affecting deconvolution accuracy. In order to apply the four distinct depth-dependent PSFs to the continuous Z-axis of data encompassing the 70-micron thick coronal slice, the following strategy was used. The ' $0 \mu \mathrm{m}$ ' PSF which used no arbor shim was used to deconvolve data from 0 to 12 microns of depth. The ' $25 \mu \mathrm{m}$ ' PSF was used to deconvolve data from 13 to 37 microns of depth. The ' $50 \mu \mathrm{m}$ ' PSF was used to deconvolve data from 38 to 62 microns of depth. Finally, the ' $75 \mu \mathrm{m}$ ' PSF was used to deconvolve data from 63 to 70 microns of depth.

Each channel of deconvolved data was then loaded into Bitplane Imaris, an advanced microscopy analysis tool which provided the functionality of volumetrically rendering imaging data. Within Imaris, the large cortical columns were subdivided into small volumetric objects for each fluorescent channel, each with a maximum size of [ $5 \mu \mathrm{m} \times 5 \mu \mathrm{m} \times 5 \mu \mathrm{m}]$. Each of these small objects has a unique centroid and is assigned to a unique depth bin. Depth bins are organized from the cortical surface to the grey/white interface in $50 \mu \mathrm{m}$ increments. By calculating the overall volume of each depth bin and the total volume of all volumetric objects assigned to the bin, it becomes simple to calculate the volume fraction of the volumetrically modeled data as a percent of the total volume of each bin. Furthermore, to account for the curvature of the cortical surface, each surface was approximated by a five-point profile. This profile was used to adjust the 'depth' of each volumetric object based on its location along the width of the cortical column. Correction of the cortical surface profile and the subsequent analysis of the cortical depth and volume of each volumetric object allows for the calculation of fractional volume as a unique percentage every $50 \mu \mathrm{m}$ from the cortical surface to the grey/white interface, for each fluorescent channel.

Subsequent statistical analysis for each of the four fluorescently labeled components is focused on two specific analyses, depth-independent and depth-dependent analyses. In the depth-independent analyses, a single fractional volume was extracted from each entire cortical column by dividing the total volume of modeled components by the total volume of the entire cortical column. For depth-dependent analysis, the fractional volume is independently extracted in 50 micron increments of depth through the cortical column. A normality test was performed on the data sets prior to each statistical analysis in order 
to select the correct methodology. A summary table of the underlying strategy is shown in Table 3.1

below. The first analysis used assesses whether a significant difference exists between paired hemispheres. This is carried out independently for each functional region, with each using 18 matched-pair slices. The second analysis examines whether a significant difference exists between the three brains. This is once again carried out separately for each functional region, with each contributing 6 values in each of three brains. The final analysis carried out examines whether a significant difference exists between the M1 and S1BF functional regions. This experiment uses the data from all 36 cortical columns, as 18 belong to each functional region. Depth-dependent analyses follow the same analysis pattern, but statistical tests are run at each unique cortical depth increment.

\begin{tabular}{|l|l|l|l|}
\hline & Normal test & Non-Normal Test & Sample Size \\
\hline Paired Hemisphere Comparison & Paired T-Test & Wilcoxon Signed-Rank & 18 matched-pairs \\
\hline Brain Comparison & ANOVA & Kruskal-Wallis & Three groups, six samples per group \\
\hline Functional Region Comparison & Unpaired T-Test & Mann Whitney U & 18 samples per group \\
\hline
\end{tabular}

Table 3.1: Summarized Statistical Testing Routine. For both depth-dependent and depth-independent analysis, the same three categories of analysis were performed. Normality testing determines whether the normal or non-normal test types are used.

Additionally, as nuclei were relatively well-dispersed, it became possible to characterize the actual density of nuclei in addition to the fractional volume assessments performed previously. This assessment was carried out in order to compare the nuclei density and nuclei volume fraction profiles, as well as to compare the results to existing literature data. Furthermore, using the total astrocytic volume per depth bin in conjunction with literature values for average Astrocyte volume, it became possible to estimate the density of Astrocytic cells throughout the depth of the cortical column. However, this is simply an estimate as it does not take into account any variability in astrocyte volume that is dependent on cortical depth.

\section{Results}

Using the depth-dependent PSF collection strategy, the PSFs from the 60x objectives of both the Nikon C2+ and Olympus FV1000 confocal microscopes were characterized and compared. Furthermore, the degree to which these PSFs were wavelength and depth-dependent was identified, demonstrating that the axial $(Z)$ length of the PSF grows due to both red-shifting the imaging wavelength as well as imaging deeper into the RI-controlled imaging chamber. This is demonstrated below in Figure 3.1. 


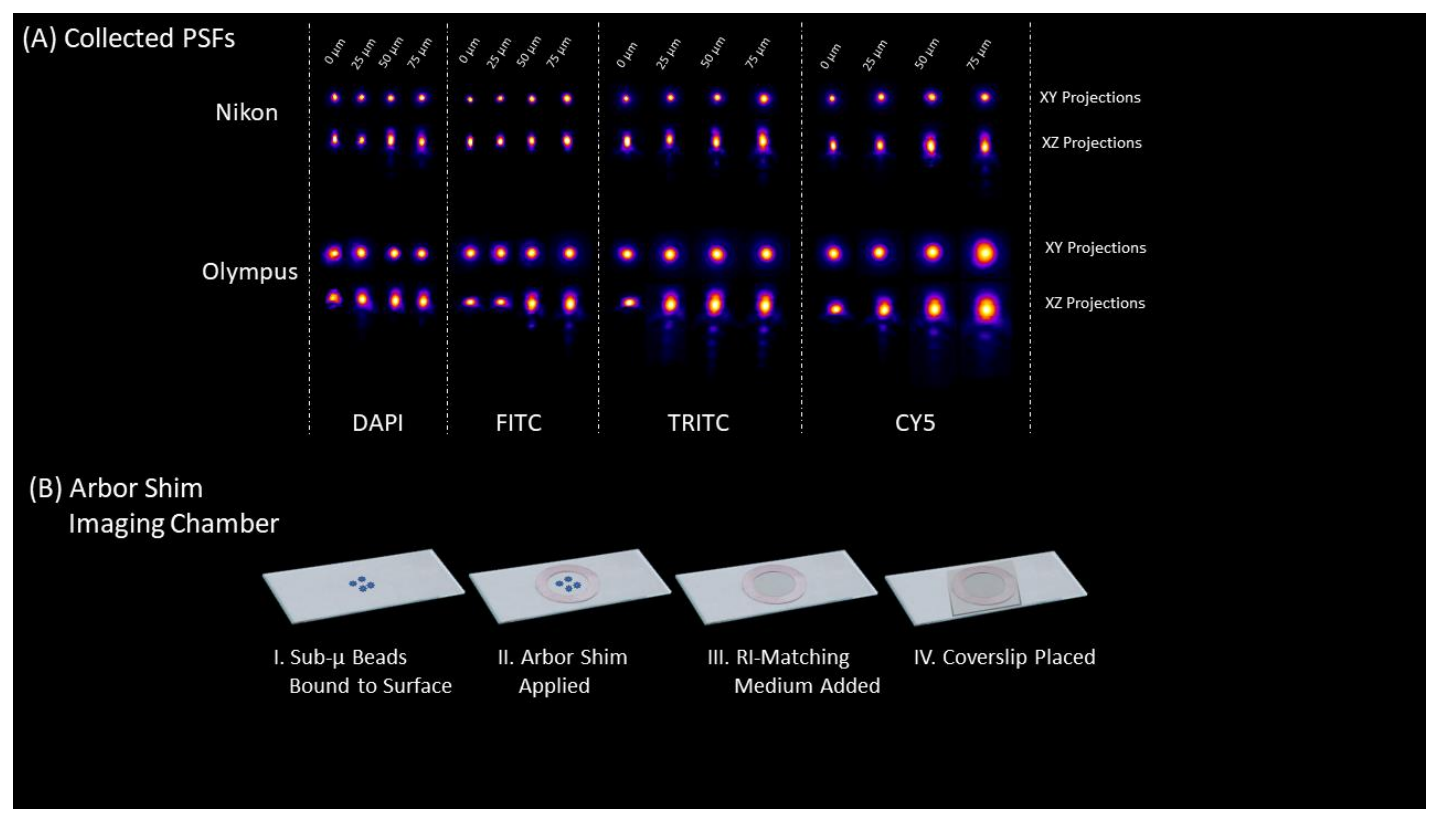

Figure 3.1: Depth-Dependent PSF Collection and Sample Chamber Construction (a) Collected point spread functions are presented as both $X Y$ and $X Z$ projections from each microscope, each fluorescent imaging channel and each specific Z-depth. The pinhole is set to the smallest diameter available in the software, 0.9 Airy Units. Displayed PSFs are an average of five collected PSFs for each condition. Note the typical enlargement of PSF which accompanies a deeper imaging condition or a more red-shifted fluorescent channel. (b) Measurement of these PSFs using a constructed sample chamber composes a four-step process. After the fluorescent beads are adsorbed to the glass slide surface, an arbor shim of defined thickness $(25,50$ or $75 \mu \mathrm{m})$ is placed on the glass slide and adhered using nail polish around the edges. A medium emulating the refractive index of brain tissue is added, and a final coverslip is adhered to the top using nail polish. Imaging of these sub-micron fluorescent beads simulates the PSF at defined depths within cortical brain tissue.

These collected PSFs, in conjunction with blind deconvolution, substantially reduced the Z-

stretching present in the datasets caused by spherical aberration. This strategy was extensively tested with simulated data with a known ground truth and varying levels of noise in order to validate the deconvolution accuracy. Figure 3.2 below highlights the impact of deconvolution on the neuronal data.

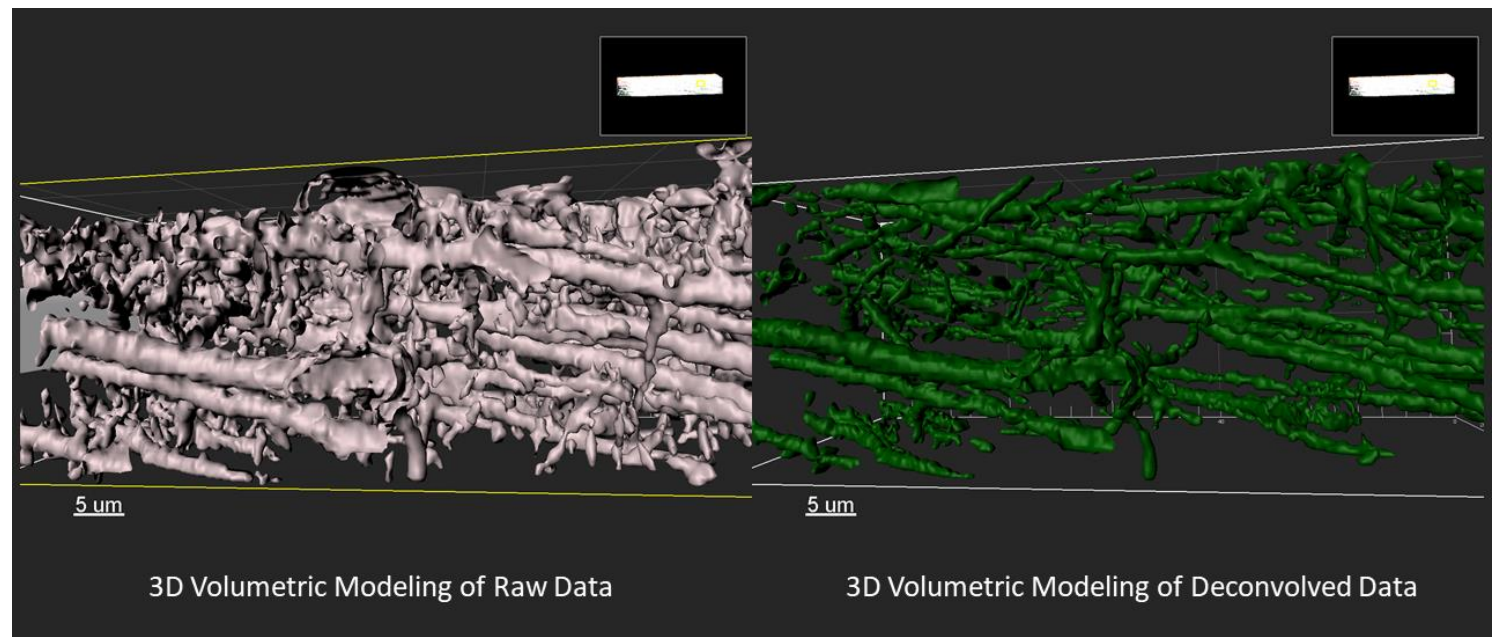

Figure 3.2: Impact of Depth-Variant Deconvolution on Data Quality. These side-by-side images demonstrate the substantial impact of depth-variant deconvolution scheme on the resultant data quality. The provided XZ projections were created through 
volumetric modeling of the raw and deconvolved image stacks in Bitplane Imaris. Note that deconvolution reduces the Z-stretching of reconstructed filaments driven by spherical aberration.

After carrying out subsequent volumetric rendering and analysis of fractional volume, the data underwent a thorough statistical analysis scheme. The general scheme is shown in Table 3.2, encompassing both depth-independent and depth-dependent analysis. A summary of statistically significant findings is also provided, highlighting that in the vast majority of cases the data was relatively homogeneous between hemispheres, between rats and between functional regions.

\begin{tabular}{|c|c|c|c|c|c|}
\hline & \multirow[b]{2}{*}{ Test Performed } & \multicolumn{2}{|r|}{ Smi-311 } & \multicolumn{2}{|r|}{ GFAP } \\
\hline & & Outcome & Notes & Outcome & Notes \\
\hline \multirow{3}{*}{ 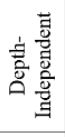 } & Hemispheric Comparison & NSD & $\mathrm{N} / \mathrm{A}$ & NSD & $\mathrm{N} / \mathrm{A}$ \\
\hline & Brain Comparison & NSD & $\mathrm{N} / \mathrm{A}$ & NSD & $\mathrm{N} / \mathrm{A}$ \\
\hline & Fx Region Comparison & SD & $\operatorname{S1BF}(25.46 \%)>\operatorname{Ml}(18.52 \%)$ & NSD & N/A \\
\hline \multirow{3}{*}{ 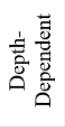 } & Hemispheric Comparison & NSD & $\mathrm{N} / \mathrm{A}$ & NSD & $\mathrm{N} / \mathrm{A}$ \\
\hline & Brain Comparison & SD & $\mathrm{SD}$ in $\mathrm{M} 1$ region $(50-300 \mu \mathrm{m})$ & NSD & $\mathrm{N} / \mathrm{A}$ \\
\hline & Fx Region Comparison & $\mathrm{SD}$ & $\mathrm{S} 1 \mathrm{BF}>\mathrm{M} 1(350-600 \mu \mathrm{m} \& 1100-1300 \mu \mathrm{m})$ & $\mathrm{SD}$ & $\mathrm{Ml}>\mathrm{S} 1 \mathrm{BF}(0-100 \mu \mathrm{m} \& 2250-2300 \mu \mathrm{m})$ \\
\hline & & \multicolumn{2}{|r|}{ VWF } & \multicolumn{2}{|r|}{ Nuclei } \\
\hline \multirow{4}{*}{ 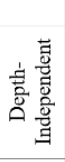 } & Test Performed & Outcome & Notes & Outcome & Notes \\
\hline & Hemispheric Comparison & NSD & N/A & $\mathrm{SD}$ & $\mathrm{SD}$ in $\mathrm{S} 1 \mathrm{BF}$ region \\
\hline & Brain Comparison & $\mathrm{SD}$ & $\mathrm{SD}$ in $\mathrm{M} 1$ region & NSD & $\mathrm{N} / \mathrm{A}$ \\
\hline & Fx Region Comparison & NSD & $\mathrm{N} / \mathrm{A}$ & NSD & N/A \\
\hline \multirow{3}{*}{ 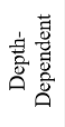 } & Hemispheric Comparison & NSD & N/A & NSD & $\mathrm{N} / \mathrm{A}$ \\
\hline & Brain Comparison & NSD & $\mathrm{N} / \mathrm{A}$ & NSD & N/A \\
\hline & Fx Region Comparison & NSD & N/A & NSD & $\mathrm{N} / \mathrm{A}$ \\
\hline
\end{tabular}

Table 3.2: Summarized Outcomes of Statistical Testing on Volume Fraction Data. Statistical testing was performed on data from each fluorescent channel. Analyses were subdivided into depth-independent and depth-dependent analyses. Depth-independent analyses utilize a single 'average fractional volume' to represent each entire cortical column. Depth-dependent analyses generate a fractional volume metric for each 50- $\mathrm{m}$ increment along the entire cortical column length. Analyses conducted compare hemispheres, multiple brains and both functional $(F x)$ regions. Depth-dependent analyses are only labeled statistically significant if

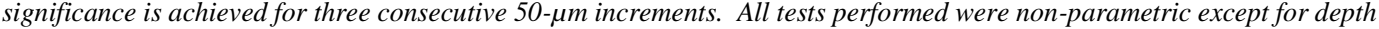
independent GFAP testing. SD (Significant Difference), NSD (No Significant Difference).

Beginning with the neuronal marker Smi-311, comparison of both functional regions in both depth-independent and depth-dependent analysis reveals that S1BF region has a significantly higher fractional volume, with depth-dependent analysis identifying this occurring primarily from 350-600 $\mu \mathrm{m}$ and 1100-1300 $\mu \mathrm{m}$. This is highlighted in Figure 3.3 below, which identifies fractional volume of Smi-311 throughout the cortical column. 
Smi-311 Staining: Fractional Volume Comparison

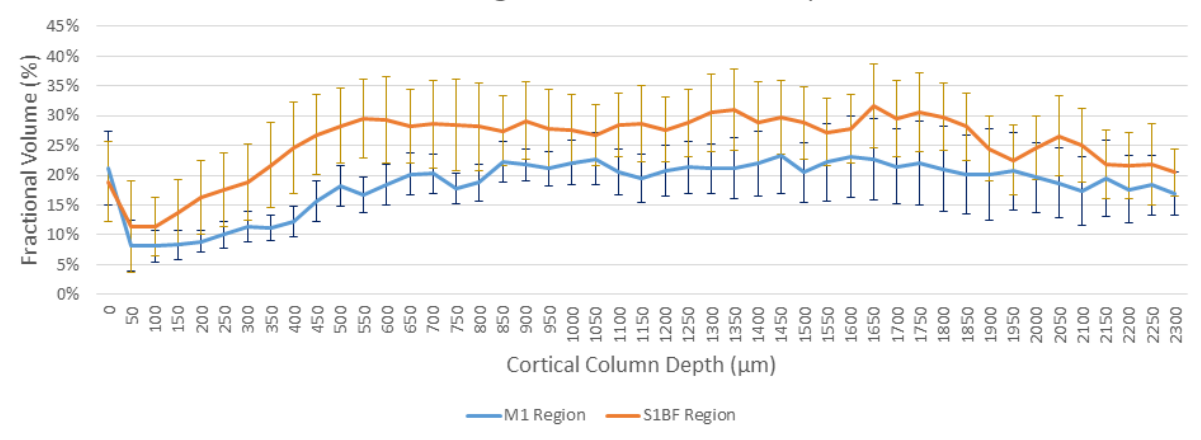

Significance of Difference between Functional Regions along Cortical Depth

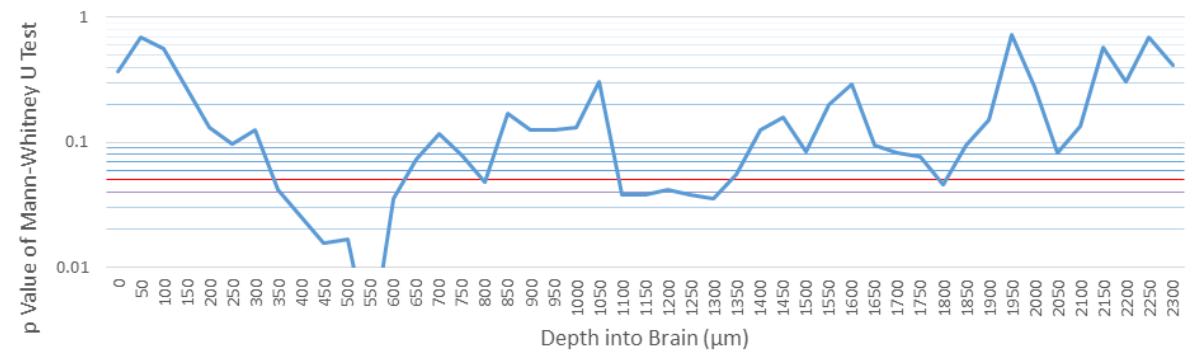

Figure 3.3: Comparison of Fractional Volume between Regions using Smi-311 (Neuronal) marker. Smi-311 fractional volume distribution along the cortical column in conjunction with the presented p-value distribution demonstrate not only that the S1BF has a typically higher density of neuronal dendritic processes, but also that statistically significant differences primarily exist in two specific ranges $-350-600 \mu \mathrm{m}$ and 1100-1300 $\mu \mathrm{m}$. Error bars on top fractional volume plot represent standard error of each data point ( $n=18$ for each functional region). The bottom plot is a log-scale representation of the p values for the depth dependent statistical comparison of $F V$ of both functional regions from the top plot, with $\alpha=0.05$ threshold highlighted with a bright red line.

Assessment of the astrocytic marker GFAP, reveals almost no statistically significant differences between the two functional regions, apart from higher GFAP fractional volume at the surface and the deepest grey/white interface regions. This is highlighted below in Figure 3.4. Furthermore, using literature estimates of GFAP-stained astrocyte volume (Bushong et al., 2002), an estimate of the astrocyte density per cubic millimeter is provided as a secondary Y scaling axis. These authors provide the critical information of the average volume of a dye-filled astrocyte in conjunction with the ratio between the volume of a dyefilled astrocyte and a GFAP-stained astrocyte (15\%), allowing usage of aggregated volume information to estimate cell density. 
GFAP Staining: Fractional Volume Comparison

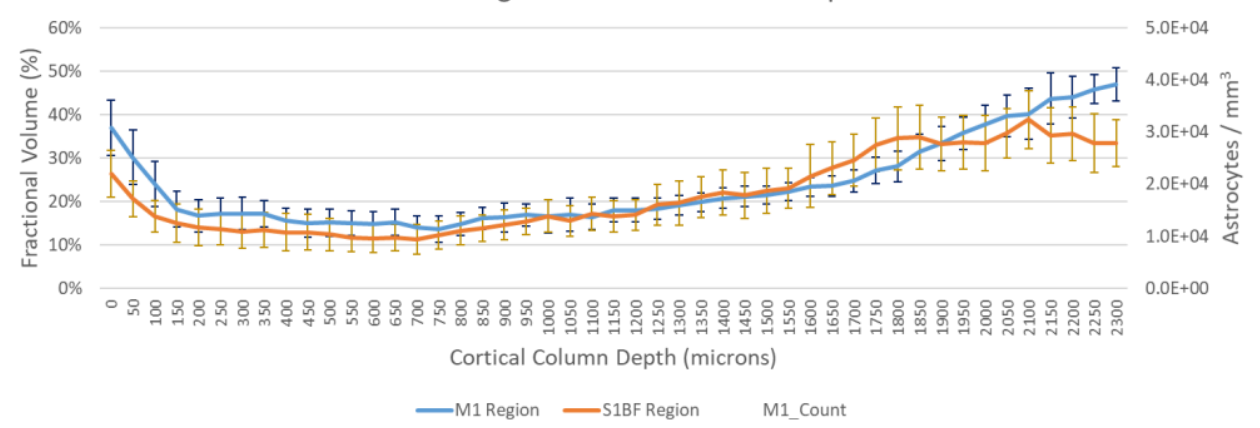

Significance of Difference between Functional Regions along Cortical Depth

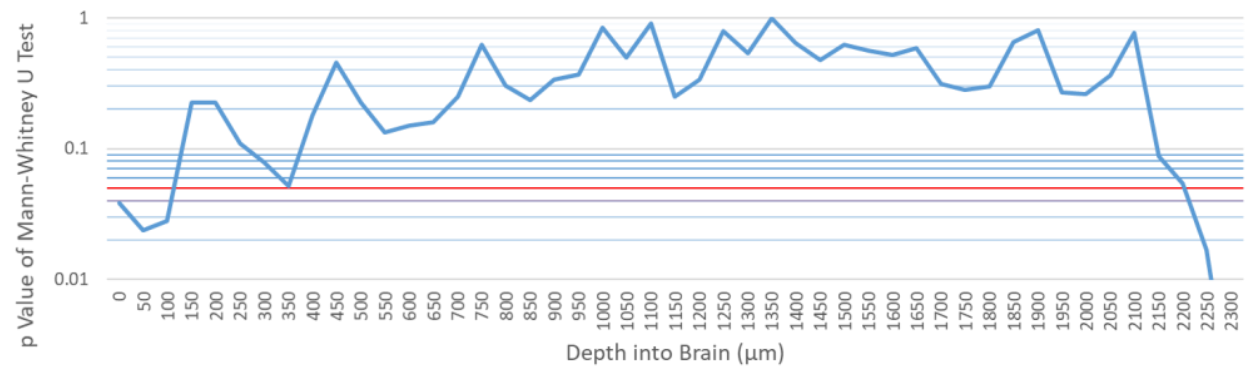

Figure 3.4: Comparison of Fractional Volume between Regions using GFAP (Astrocyte) marker. GFAP fractional volume distribution along the cortical column in conjunction with the presented p-value distribution demonstrate statistically significant differences in two specific ranges - 0-100 $\mu \mathrm{m}$ and 2200-2300 $\mu \mathrm{m}$. Error bars on top fractional volume plot represent standard error of each data point ( $n=18$ for each functional region). Furthermore, an additional $y$-scaling on the right side of the plot estimates the Astrocyte density $/ \mathrm{mm}^{3}$ based on literature estimates of GFAP-stained astrocyte volume (McCaslin et al., 2011). The bottom plot is a log-scale representation of the $p$ values for the depth dependent statistical comparison of $F V$ of both functional regions from the top plot, with $\alpha=0.05$ threshold highlighted with a bright red line.

Analysis further reveals that the vascular marker, VWF, has no substantial difference between the motor and somatosensory regions. This is highlighted below in Figure 3.5. 
VWF Staining: Fractional Volume Comparison

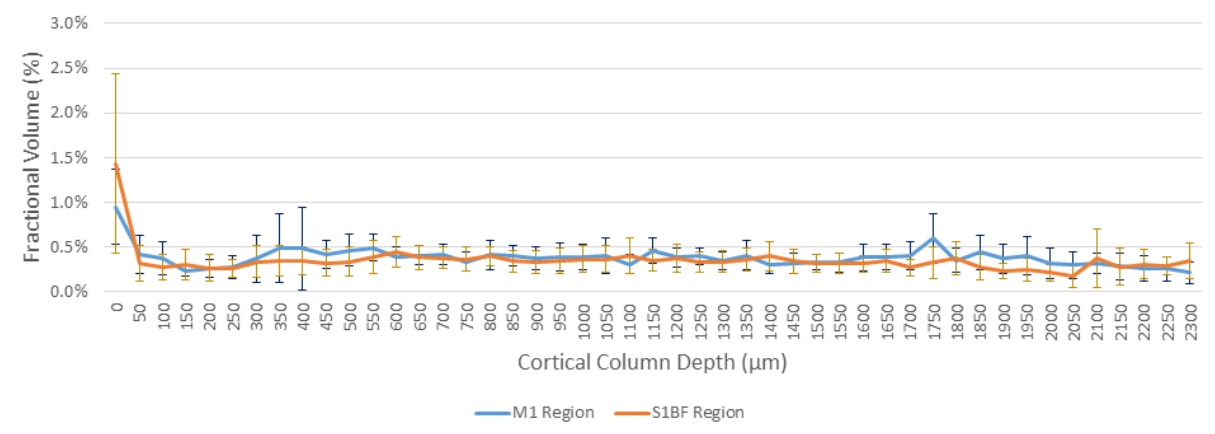

Significance of Difference between Functional Regions along Cortical Depth

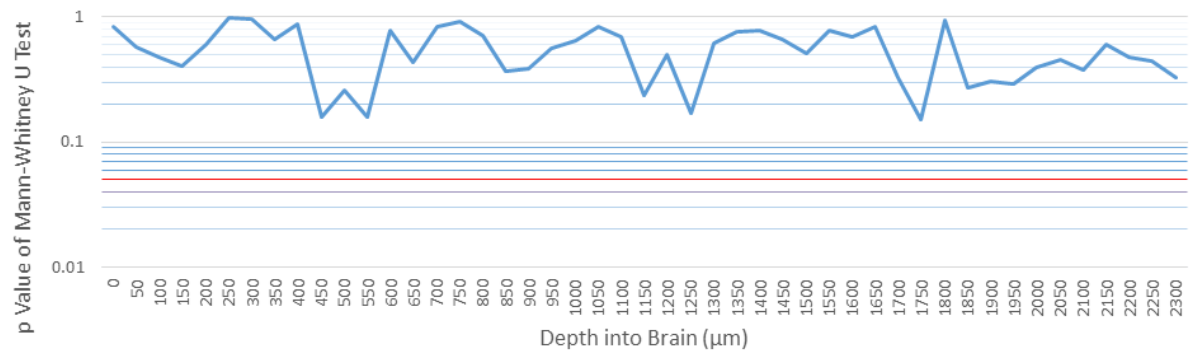

Figure 3.5: Comparison of Fractional Volume between Regions using VWF (Vascular) marker. VWF fractional volume distribution along the cortical column in conjunction with the presented p-value distribution demonstrate no statistically significant differences between the two functional regions. Error bars on top fractional volume plot represent standard error of each data point $(n=18$ for each functional region). The bottom plot is a log-scale representation of the $p$ values for the depth dependent statistical comparison of $F V$ of both functional regions from the top plot, with $\alpha=0.05$ threshold highlighted with a bright red line.

Finally, assessment of the nuclear marker reveals no significant differences in fractional volume between the M1 and S1BF regions. This is demonstrated below in Figure 3.6. Alternatively, examinations of nuclei density similarly have no difference between the M1 and S1BF region, with the density profile appearing the same as the volume fraction profile. This is shown below in Figure 3.7. 
Nuclei Staining: Fractional Volume Comparison

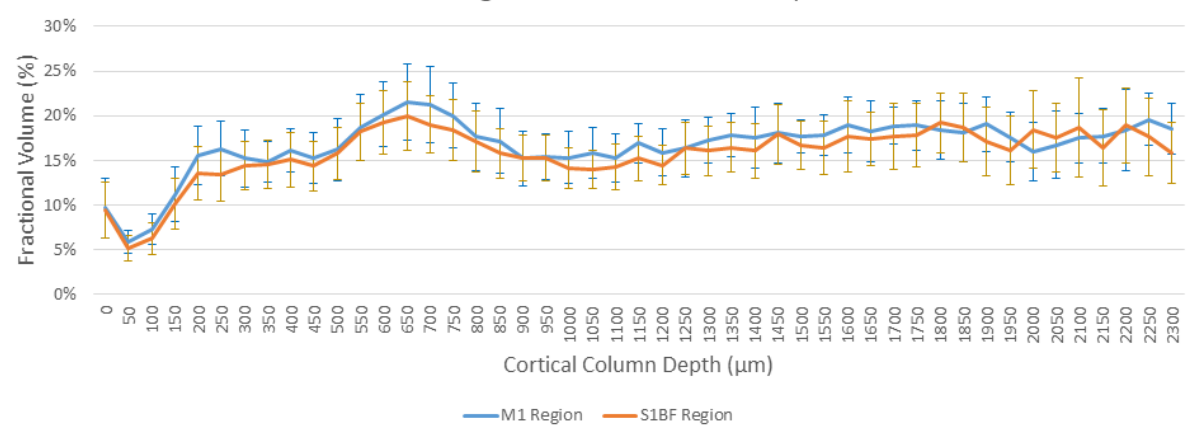

Significance of Difference between Functional Regions along Cortical Depth

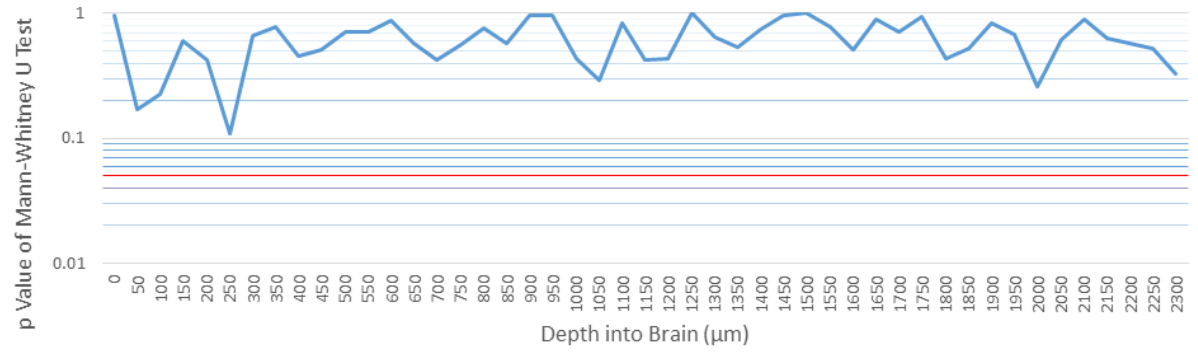

Figure 3.6: Comparison of Fractional Volume between Regions using Nuclei marker. Nuclei fractional volume distribution along the cortical column in conjunction with the presented p-value distribution demonstrate no statistically significant differences between the two functional regions. Error bars on top fractional volume plot represent standard error of each data point ( $n=18$ for each functional region). The bottom plot is a log-scale representation of the $p$ values for the depth dependent statistical comparison of $F V$ of both functional regions from the top plot, with $\alpha=0.05$ threshold highlighted with a bright red line.

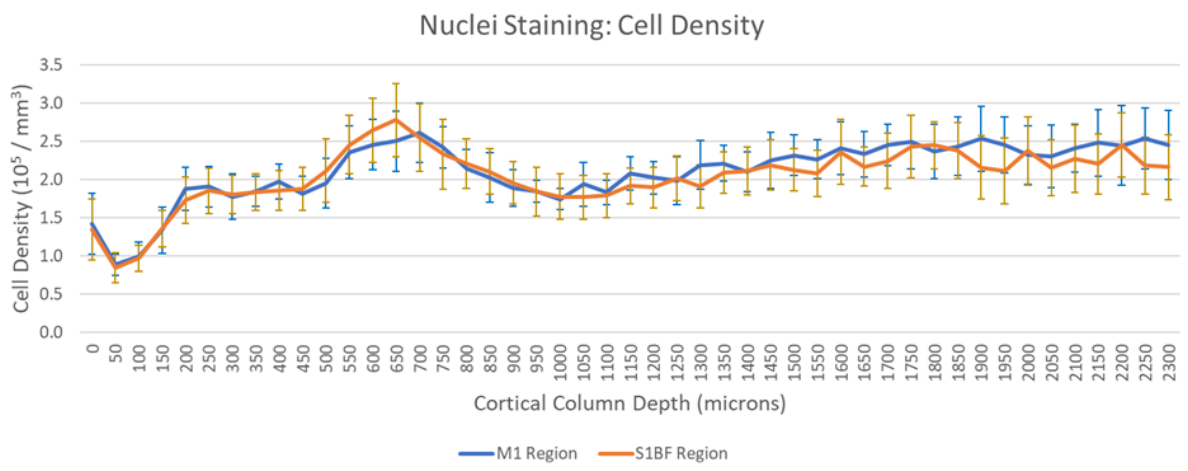

Significance of Difference between Functional Regions along Cortical Depth

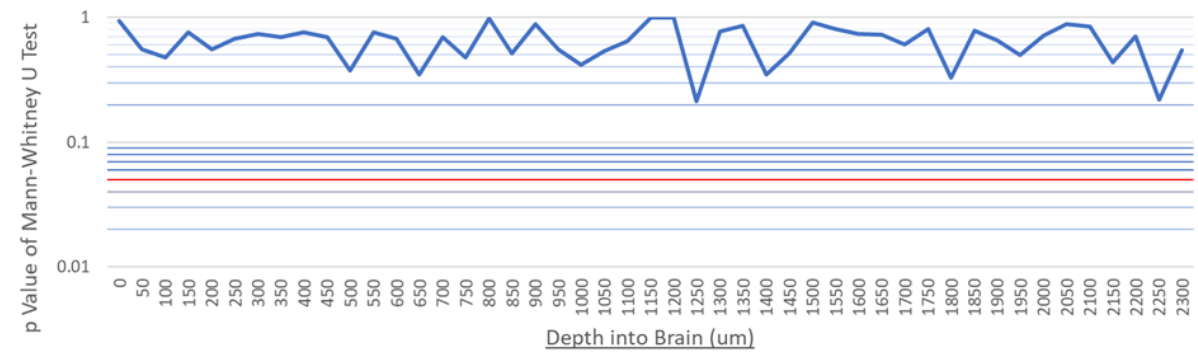

Figure 3.7: Nuclei Density along Cortical Column. Nuclei cellular density along the cortical column in conjunction with the presented p-value distribution demonstrate no statistically significant differences between the two functional regions. Error bars on top fractional volume plot represent standard error of each data point ( $n=18$ for each functional region). The bottom plot is a logscale representation of the $p$ values for the depth dependent statistical comparison of FV of both functional regions from the top plot, with $\alpha=0.05$ threshold highlighted with a bright red line. 
In order to understand the dense interconnectivity of these cell types in the neuropil, it is useful to have a visualization of the rendered data. This is provided below in Figure 3.8.

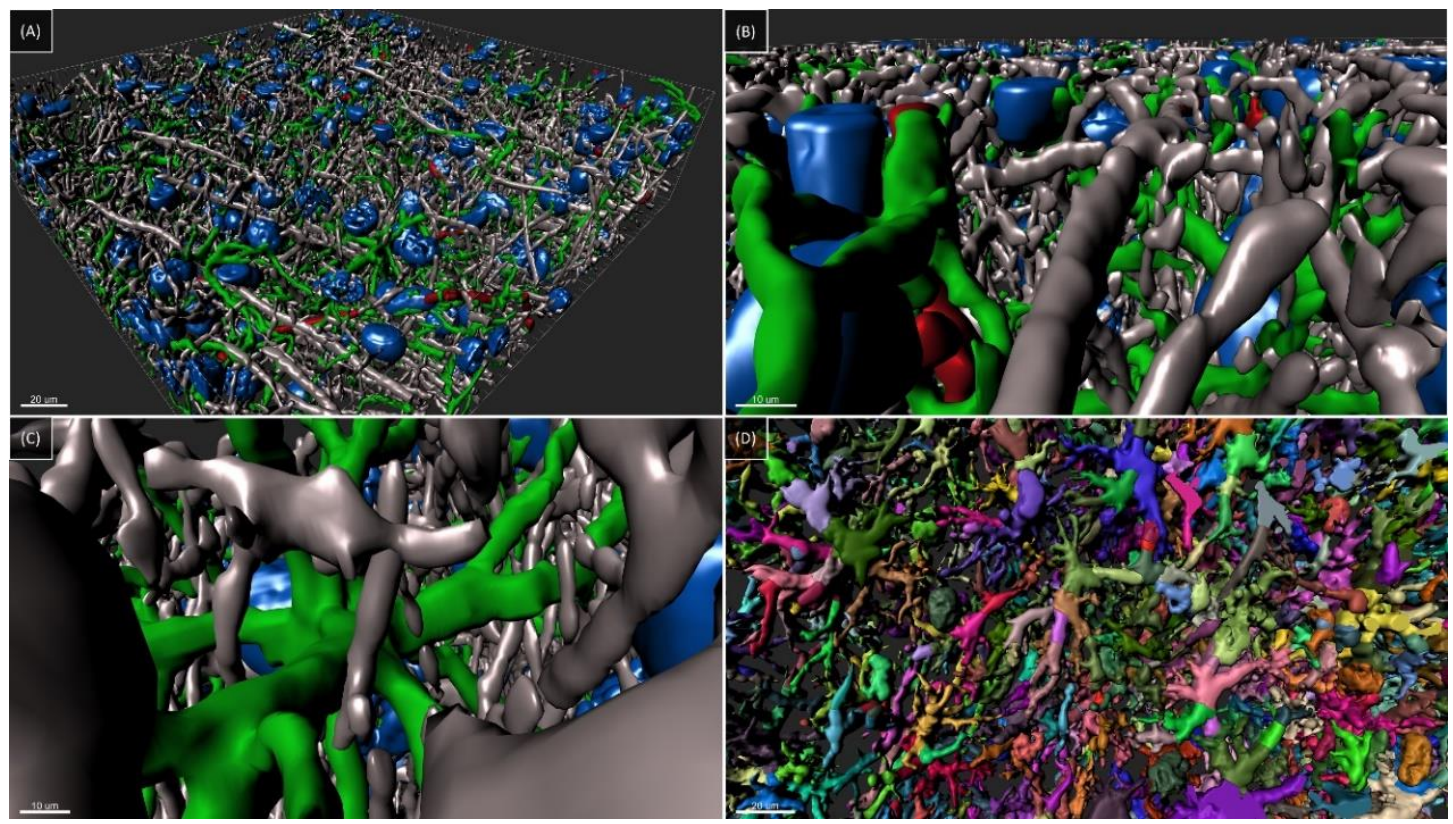

Figure 3.8: Visualization of Interconnectivity of Stained Cellular Markers. Architectural interconnectivity of the neuronal dendrites (grey), astrocytes (green), vasculature (red) and nuclei (blue) can be best visualized through $3 D$ modeling of the neuropil. $(A, B) T w o$ perspectives of the outer surface of a small sub-cube extracted from the cortical column reveals the neuropil's intrinsic complexity. (C) Within this dense data cube, an astrocyte is shown flanked by numerous dendritic processes. (D) The underlying process of fractional volume analysis is provided here, demonstrating for the astrocyte channel how the data cube (and individual astrocytes) are subdivided into numerous smaller volumetric objects - randomly pseudocolored for ease of visualization. Extraction of the positional and volumetric characteristics of each individual fragment allows for continuous measurement of volume fraction along the cortical column.

\section{Discussion}

A variety of methodological advances and useful quantitative metrics for the motor and somatosensory regions of the murine brain are generated which can be leveraged by future research groups. Th simple methodology developed for generating depth-variant PSFs and subsequent deconvolution provides a valuable protocol for labs to enhance the accuracy of their 3D data cubes. Furthermore, by developing depth-independent and depth-dependent fractional volume metrics for neuronal, astrocytic, vascular and nuclear components in healthy adult murine tissue, foundational metrics are provided which can be used towards computational studies, disease state characterization and better characterization of MRI quantitative metrics in techniques such as quantitative gradient recalled echo (qGRE) (Wen et al., 2018) and diffusion MRI (Gilani et al., 2019). Usage of these techniques can take advantage of wellunderstood changes in fractional volume, such as increases in local vascularization due to tumor 
angiogenesis (Pathak et al., 2001). The applications of this work in predicting local fractional volumes of major cellular components using MRI is especially exciting due to its long-term potential for providing a noninvasive estimation of the severity of disease states that may currently require a risky biopsy of brain tissue. Additionally, characterization of Astrocytic volume fraction in 50 micron increments along the cortical depth of the M1 and S1BF region can hence provide a useful map of depth-dependent variations of glutamate uptake in these regions (Medvedev et al., 2014).

While few direct comparisons exist in the literature for many of the analyses performed here, examples exist that support some key findings. In regard to the neuronal Smi-311 marker, this study identified a significantly higher volume fraction in the S1BF compared to the M1 region in a specific region of the cortical column. Neuron density has been established as higher in the S1BF region as compared to the M1 region in both primates (Young et al., 2013) and mice (Herculano-Houzel et al., 2013; Keller et al., 2018), although these authors are unable to identify the specific cortical depth where these differences become significant. Similarly, other authors have identified that the neuron staining marker Rorb is expressed at a lower intensity in the motor region of mice as compared to the sensory region. Considering the astrocytic GFAP stain, there are very few examples for comparison in the scientific literature. However, one group characterizing astrocyte density continuously through the somatosensory cortex of a mouse identified a similar trend of an early peak in superficial layers, a dip in intermediate layers and a rapid rise when approaching the grey/white interface (McCaslin et al., 2011). This dissertation's approach utilizes fractional volume while the literature example utilizes astrocyte density, but they both demonstrate similar depth-dependent profiles. Another group of authors provided useful metrics as to the average dye-filled astrocyte volume as well as the ratio of this to a GFAP-stained astrocyte (15\%) (Bushong et al., 2002). Using this knowledge and the aggregated volume information collected in this study, the Astrocyte density curve from (McCaslin et al., 2011) was replicated in Figure 3.5 and surprisingly nearly perfectly matched the absolute density found in the literature - ranging from $1 \cdot 10^{4}$ to $4 \cdot 10^{4}$ astrocytes $/ \mathrm{mm}^{3}$ along the cortical column. Assessing the VWF analysis, related work exists which characterizes fractional volume as a function of cortical depth in mice (Tsai et al., 2009) as well as work which simply summarizes an 'average' fractional vascular volume (L.-Y. Zhang et al., 2018). The results of this study match the general shape of the literature fractional volume profile. However, it is important to 
mention that the absolute measurements of vascular fractional volume calculated in this study underestimate of the true fractional volume as the VWF stain does not provide as continuous of a vascular tracing as alternative staining markers such as RECA-1. RECA-1 was not available for this study due to limitations on available animal antibody sources resulting from using four simultaneous fluorescent markers. Finally, considering the nuclear staining fractional volume, the distinct profile seen from the results of this study well matches various literature representations of continuous cell density along the cortical depth (Wu et al., 2016) (Keller et al., 2018). Furthermore, assessment of absolute nuclei density from this study has a Y-axis ranging from $1 \cdot 10^{5}-3 \cdot 10^{5}$ cells $/ \mathrm{mm}^{3}$, which closely matches the range seen in both of these literature examples. In conclusion, these various examples from the literature provide clear evidence as to the validity of the findings.

This work also provides additional evidence towards a theory of cerebral cortex evolution that intertwines the wire volume minimization principle and the spine proportion economical maximization principle (Karbowski, 2015). These theories encompass a general principle that axons and dendrites each compose $1 / 3$ of cortical volume, astroglia compose $(1 / 3)^{2}$ of cortical volume and capillaries compose $(1 / 3)^{4}$ of cortical volume. According to this evolutionary theory, this ratio is believed to be generally conserved regardless of brain size or functional region. These hypotheses generally fit the themes of the presented findings in relation to both the conservation of fractional volume across brain and functional region as well as the diminishing fractional volume when moving from neuronal to astrocytic and finally vascular components.

Furthermore, this work contributes to recent research foci examining the motor cortex as a potentially 'pseudo-granular' region instead of its traditional labeling as an agranular region. Historically, the motor cortex is considered agranular due to its lack of traditional layer IV cytoarchitecture, which is believed to be lost postnatally (Barbas \& García-Cabezas, 2015). The unique cytoarchitecture of Layer IV in granular regions of the brain is typically reflected by the presence of dense granular neurons, a neuronal subtype characterized morphologically by a small soma and a few small claw-like dendritic projections (Rizzolatti \& Luppino, 2001). While the motor cortex lacks this cellular component, evidence has emerged that Layer IV still exists in the motor cortex in a connectivity context, since it still receives input from primary thalamocortical axons and provides output to excitatory neurons in Layers II and III, both key 
characteristic of Layer IV in granular tissue (Yamawaki et al., 2014)(Bopp et al., 2017). The analysis presented in this study further contributes to this discussion by demonstrating no significant difference in nuclei staining density or fractional volume between the 'agranular' motor and 'granular' somatosensory regions - an unexpected event due to the expected difference in nuclei density due to the lack of a dense granular cell layer. Since granular and agranular cortices have been demonstrated as having very different nuclei density profiles in the mouse (Tsai et al., 2009), the presented research provides additional evidence of the underlying cytoarchitectural similarities between the motor and somatosensory regions.

Furthermore, a statistically significant decrease in neuronal staining volume is identified in this study in the region corresponding to Layer IV (500-600 microns deep) of the motor versus somatosensory cortex, which matches previous work in the literature examining the expression of the neuronal marker Rorb in the motor and somatosensory cortices (Yamawaki et al., 2014). The identification of the Layer IV region is performed based on aligning the layer-specific cell density profiles provided in previous literature (Tsai et al., 2009) (Keller et al., 2018) (Wu et al., 2014) with the experimentally calculated cell density profile.

Despite the exciting applications of the developed work, there are a number of key limitations which must be addressed. First, FVA does not provide metrics which can be used to understand individual cell morphologies nor functional activity in brain tissue. Furthermore, while the blind deconvolution methodology can enhance the validity of FVA metrics by reducing axial elongation that is introduced during the imaging process, deconvolution carries the risks of creating additional artifacts in the final data. While this is minimized by limiting the number of deconvolution iterations to 10 and reducing shot noise in the dataset through median filtering prior to deconvolution, the risk is still present. Finally, estimates of astrocyte density made using the fractional volume data does not take into account the potential heterogeneity of astrocyte form throughout the cortical column, which limits the accuracy of this particular estimation method. 


\section{Chapter 4. Automated Nuclei Classification \& Astrocytic Individualization}

\section{Introduction}

Characterizing the morphological properties of individual cells is a fundamental goal within the broader aim of understanding the complex architecture of the cerebral cortex. Due to the density of interwoven filaments in the neuropil, this can be an extremely difficult challenge. An important starting point in this form of analysis is a clear identification of each cell and its nucleus, so that morphological analysis can utilize the cell center correctly during filament tracing. This can be readily accomplished by using a combination of fluorescent structural cell-specific markers and a fluorescent generalized nuclear marker, since linking of a nucleus to an individual cell becomes simpler due to the unstained 'hole' within the cell soma where the nucleus lies. On a smaller scale, this technique is appropriate, since subsequent manual filament tracing can effectively extract 3-dimensional cell structure. However, for processing large datasets with tens of thousands of nuclei, the development of an automated approach becomes necessary.

While the underlying goal of automated nuclei classification has clear advantages in a variety of common fluorescence microscopy scenarios, its implementation has not yet been realized in the academic literature. The most similar example is a project which correlated neuronal and microvascular density in the murine cortex through automated mapping of the location of neuronal and non-neuronal nuclei (Tsai et al., 2009). This group identified neuronal (and non-neuronal) nuclei based on the colocalization of the neuronal nuclei marker NeuN and the generalized nuclei label DAPI - two markers whose fluorescent signals were well-aligned for neuronal nuclei. Interpretation of colocalized fluorescent labeling has also been used to distinguish between different subtypes of neurons due to labeling of unique transcription factors (Sigl-Glöckner \& Brecht, 2017), but this technique is even more distantly related to the concept of cell-specific nuclei assignment.

One of the closest-matching implementations of cell-specific nuclei assignment was carried out without fluorescence - instead using assessment of cytological features following Nissl staining (GarcíaCabezas et al., 2016). The methodology required manual assignment of each nuclei by experienced raters, which although lacking the benefits of automation provided a reliable identification with an inter-rater 
reliability (Krippendorff's alpha) value of 0.92 . The cytological identification relied on the darkness of the nuclei stain, its shape, the presence of cytoplasm and the distribution of nuclear heterochromatin.

Despite the rarity of nuclei assignment techniques in the literature, they hold tremendous promise for the detailed morphological assessment of cortical astrocytes. Cortical astrocytes in the dense neuropil connect at their peripheral end-feet, which makes their individual volumetric rendering and analysis extremely difficult during typical immunofluorescent staining. The complexity of astrocyte individualization is highlighted in Figure 4.1. Researchers attempting to analyze individual astrocyte morphology in co-cultured neuron-astrocyte networks have described that the dense entangling makes individual cell separation impossible (Bar El et al., 2018). In order to independently analyze the morphology of a single astrocyte, researchers commonly use dye-filling (Savtchenko et al., 2018) (Wilhelmsson et al., 2006) (Moye et al., 2019). While this technology serves a unique niche, it suffers from being extremely time-consuming and requiring expensive additional instrumentation, preventing its use for automated morphological analysis of large numbers of astrocytes.

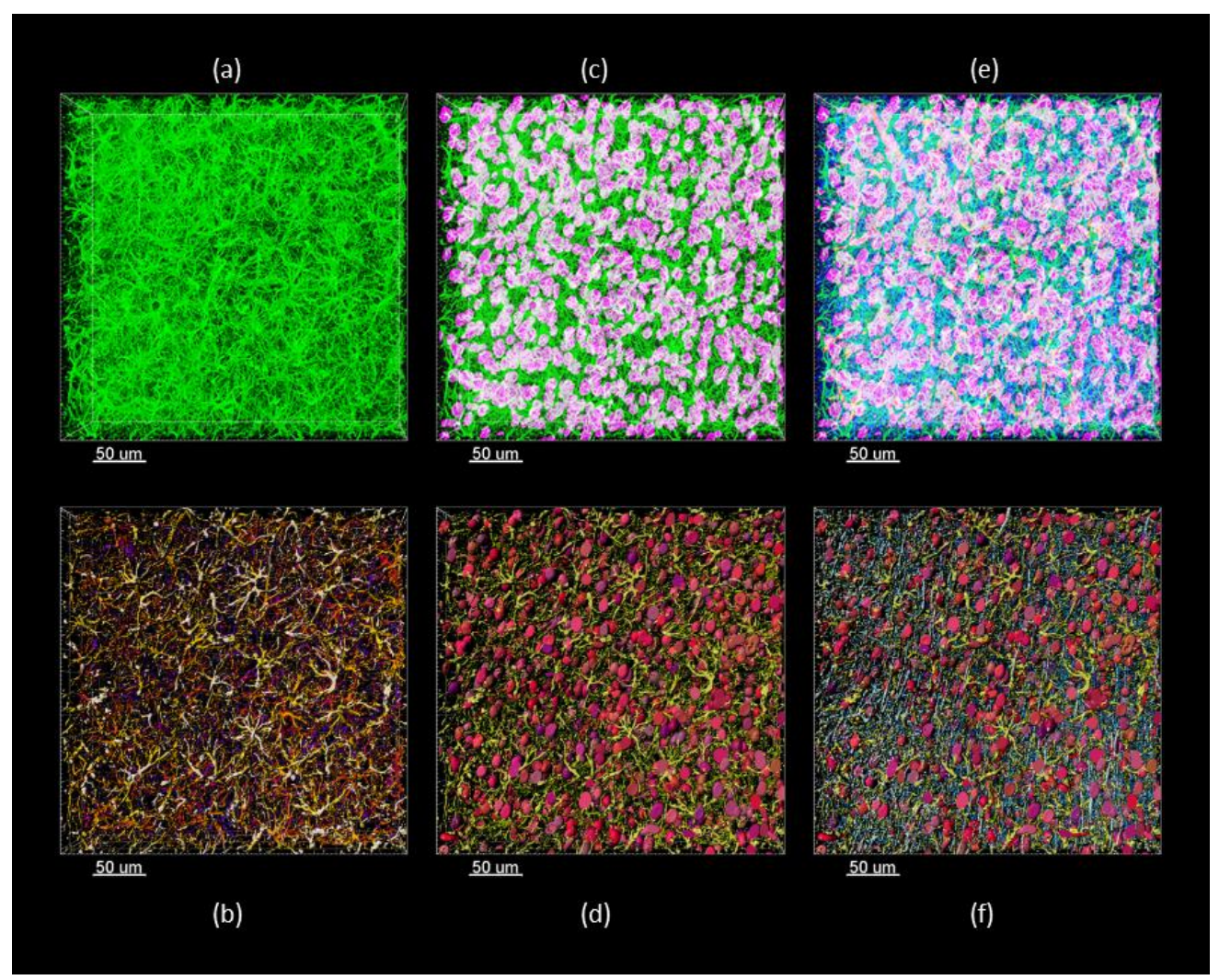

Figure 4.1: Complexity of Individual Astrocyte Identification. Astrocytes form a highly interconnected network in the dense neuropil and are extremely difficult to separate, which is demonstrated in green in the blended XY projection (a) and volumetric model (b), where filaments are pseudocolored based on Z-depth for enhanced visibility. Nuclei are then added to (a) in (c) to 
highlight the challenge of individual-astrocyte nuclear alignment, which is then volumetrically modeled in (d) with astrocytes in yellow and nuclei in randomized shades of pink/purple for ease of visibility. When the remaining two fluorescent channels (neuronal dendrites \& microvasculature) are added in the blend projection (e) and volumetric model (f), it becomes clear the immense challenge of precisely identifying individual astrocytes and their corresponding nuclei. Figures generated using Bitplane Imaris.

Apart from dye-filling, the only other currently available method for astrocyte morphological individualization is manual process tracing. Using the Fiji-ImageJ Simple Neurite Tracer (SNT) plugin, one group demonstrated the capability to measure process thickness, process length and carry out Sholl's analysis of rodent astrocytes (Tavares et al., 2017). Another group used the same plugin to demonstrate a cortical-layer specific territorial volume and orientation angle of neocortical astrocytes in the mouse somatosensory cortex (Lanjakornsiripan et al., 2018). A third group used the manual tracing capability of the Neurolucida software package to distinguish the morphology of astrocytes in Macaques suffering from simian immunodeficiency virus (SIV) from a healthy control group based on Sholl's analysis, arbor volume, arbor length and the presence of bifurcations (K. M. Lee et al., 2014). Manual process tracing reduces the need for expensive microinjection equipment, but still requires extensive manual effort and is poorly suited for large-area automated morphological analysis.

Characterization of astrocyte morphological diversity has become a popular topic in recent years due to recent progress uncovering their heterogeneity and links to its unique effects on underlying neuronastrocyte spatiotemporal interplay (Zhou et al., 2019). The underlying structural plasticity of astrocytes is believed to be critical in uncovering the molecular nature of various cognitive processes, such as learning and memory (Suzuki et al., 2011)(Ben Menachem-Zidon et al., 2011). The diversity of astrocytes has been further supported through a variety modalities, including RNA sequencing and electron microscopy, but a clear definition of the diverse classes is still elusive (Khakh \& Deneen, 2019). Since the morphological diversity has a direct impact on their interaction with neuronal synapses, other glia and surrounding vasculature, further study into astrocyte morphology is critical to better understand this cell type in both normal and diseased tissue (Schiweck et al., 2018).

The most substantial contribution to aid nuclei assignment for astrocytes was the recent identification of a nuclear marker, Sox9, which is specific for astrocytes and glial progenitors. Outside of neurogenic regions, Sox9 has been revealed to be a useful astrocyte-specific nuclear marker (W. Sun et al., 2017). However, it is important to recognize that due to its parallel specificity for progenitor cells, Sox $9^{+}$ 
glia have also been found to generate glutamatergic neurons, demonstrating that subsequent interpretation of staining patterns must proceed cautiously (Kaplan et al., 2017). Since parallel Sox9 staining would require usage of an additional fluorescent channel, of which modern fluorescent microscopes typically only have three or four available, it is more beneficial to leverage a technique that can utilize a generalized nuclei fluorescent stain to identify multiple cell type categories in parallel.

The current work aims to resolve existing challenges of cell-specific nuclei identification and subsequent astrocyte morphological analysis by taking advantage of bright staining patterns in the perinuclear rim surrounding nuclei within cellular soma. A unique four-channel staining and imaging routine separately labels astrocytes, neurons, vasculature and nonspecific nuclei. Sequential steps of nuclei volumetric modeling, masking, binarization and dilation allows for unique assessment of staining density in the remaining three channels in the small region immediately surrounding each nucleus where the soma corresponding to the nuclei lies. Extraction of the perinuclear fluorescent intensity in the astrocytic, neuronal and vascular channels in conjunction with the use of a state vector machine 3D discrimination hyperplane allows for reliable distinction of relevant cellular components - specifically astrocytic, neuronal, vascular and other-cell nuclei. Parameter optimization carried out using pooled and un-pooled training/testing sets maximizes sensitivity and specificity of the resulting hyperplanes. Recombination of selected nuclei with their cell-specific fluorescent channel allows for cell individualization and subsequent surface and filamentous analysis for calculating cell-specific metrics.

The novelty of this work rests on its use of perinuclear fluorescent staining intensity of the neuronal, astrocytic and vascular channels for nuclei sorting. As a result, this methodology emerges as the first published technique for automated astrocyte identification and individualization for rapid morphological quantification. Furthermore, this technique identifies optimal perinuclear distances from the nuclear boundary to maximize sensitivity and specificity for subsequent cell-type identification. This research is highly impactful for researchers who aim to rapidly collect and analyze distinct astrocyte morphologies for subsequent assessment of their heterogenous form and function and who have a multichannel confocal fluorescent microscope 


\section{Methods \& Materials}

Details on brain collection, slicing and subsequent immunofluorescent staining and imaging can be found in Chapter 2. Briefly, adult Wistar rats were sacrificed and coronal slices $70 \mu \mathrm{m}$ thick were sectioned using a vibratome. Landmarks were identified using a rat brain atlas to identify slices containing the primary motor (M1) and primary somatosensory (S1BF) regions. From three unique rat brains, three slices each were collected containing the M1 and S1BF regions - encompassing 18 slices in total. Each slice was immunofluorescently stained with four markers. The first marker, Smi-311 (Pan-neuronal neurofilament marker), is a neuronal marker which labels dendrites and soma of all neuronal cells. The second marker, GFAP (Glial Fibrillary Acidic Protein), labels Astrocytic soma and arbors. The third marker, VWF (Von Willebrand Factor), labels the vascular endothelium. The final marker, a commercial product called NucRed Dead, is a fluorescent generalized nuclei stain.

Slices were stained and entire cortical columns from the functional region of interest in each hemisphere were imaged at 60x magnification, spanning approximately [800 $\mu \mathrm{m}$ x $3000 \mu \mathrm{m} \times 70 \mu \mathrm{m}]$ at a voxel size of $[0.207 \mu \mathrm{m} \times 0.207 \mu \mathrm{m} \times 1 \mu \mathrm{m}]$. Data was collected from two fluorescent confocal microscopes, an Olympus FV1000 and a Nikon C2+. The Olympus required two successive cortical column scans, each of two channels, while the Nikon had the intrinsic capability to collect all four channels during a single scan. Unfortunately, the need for two successive scans created spatially varying offset between each pair of collected channels in the Olympus data, requiring usage of only the collected Nikon data for this particular study until improved stitching tools could be implemented to resolve the challenges of the Olympus datasets. The data collected from the Nikon C2+ encompassed one slice from the M1 and another from the S1BF groups, providing two cortical columns from each functional region which were leveraged for this study.

To resolve issues with depth-dependent spherical aberration in each of the four channels, a unique PSF collection and deconvolution routine was implemented as discussed in Chapter 3. Briefly, using an imaging chamber filled with a liquid chosen to match the refractive index of brain tissue, unique point spread functions were collected at simulated depths of $0 \mu \mathrm{m}, 25 \mu \mathrm{m}, 50 \mu \mathrm{m}$ and $75 \mu \mathrm{m}$ for each of the four fluorescent channels. Blind deconvolution was utilized within Matlab to reduce the substantial axial 
stretching that occurs as a result of spherical aberration. Deconvolution is a necessary step to assess the perinuclear rim since PSF-driven blurring both laterally and axially can cause the nuclei to obscure the perinuclear rim - which in many cases can be only a few pixels wide at a high magnification.

Once the cortical column has been successfully acquired for all four channels and the resultant data has been stitched and deconvolved, the next step is to collect discrete metrics for each individual nucleus within the cortical column to facilitate later cell-category assignment. To accomplish this objective, Bitplane Imaris was utilized to transform the nuclei-channel data cube into a collection of volumetrically modeled nuclei. In order to ensure that nuclei with 'touching' boundaries were properly separated into distinct objects, Imaris' built-in seed-point separation tool was leveraged, which uses the watershed algorithm to split clustered nuclei. Once all nuclei were uniquely volumetrically modeled, the distinct characteristics of each were exported - including the centroid position, volume and sphericity. Furthermore, the volumetrically modeled objects were masked to generate additional image stacks - one in which all volumetric objects were binarized and another where each individual volumetric object was given a unique intensity value.

Identification of the perinuclear rim requires a sequence of simple steps. First, binarized nuclei undergo a 3-dimensional binary fill operation in order to ensure that any 'holes' present within them due to weaker interior staining are eliminated. Next, a binary dilation procedure is carried out, which expands the binarized region by a specific number of pixels from the existing boundaries of the binarized nuclei.

Finally, the dilated and un-dilated binarized nuclei are subtracted from one another, leaving only the thin ring around the nuclei representing the location of the perinuclear region. Since the binarized data has a perinuclear area with a value of 1 and a nuclear and background region with a value of 0 , the data can then be directly multiplied by the image stack of the same region in the other fluorescent channels, allowing an effective sampling of the perinuclear region staining intensity from other fluorescently stained channels. A diagram highlighting this process is shown in Figure 4.2. 


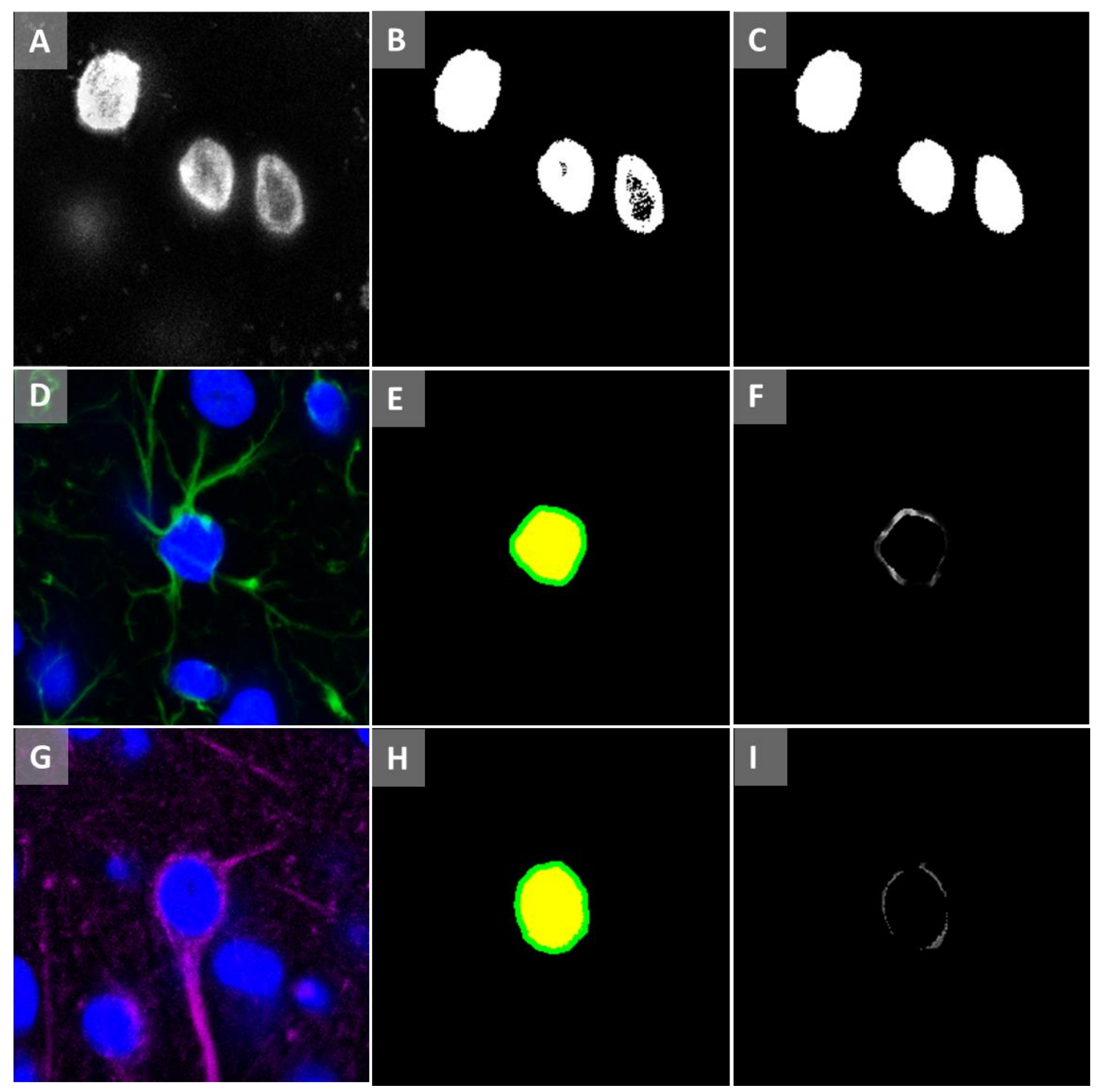

Figure 4.2: Demonstration of Perinuclear Parameter Extraction Technique. First, collected nuclei (A) are binarized (B) and then undergo a binary fill operation $(C)$ to ensure full representation of the nuclear volume. For both Astrocytes $(D)$ and Neurons $(G)$, the perinuclear zone for each cell type consists of a thin ring surrounding the nucleus. The highlighted nucleus in each case first undergoes a binary dilation operation of five pixels, creating two distinct binary objects - a yellow nucleus and green perinuclear zone (E,H). Multiplication of the binarized perinuclear zone and the corresponding cellular channel's grayscale image allows for sampling of the perinuclear zone $(F, I)$. Subsequently, the pixel values within the perinuclear zone of these images are extracted for further analysis.

In order to demonstrate that the perinuclear staining approach is valid for discriminating between different cell-type categories, it became necessary to annotate a subset of existing nuclei in the dataset so that their perinuclear staining values from each channel can be compared to the annotated cell type. To accomplish this, a Matlab-based GUI was developed to facilitate nuclei selection and annotation, the details of which are presented in Figure 4.3. Approximately 50 nuclei were collected from each of the four desired groups - 'neuron', 'astrocyte', 'vasculature' and 'other cell'. The GUI accepts as input the nuclei-specific 
statistics from Imaris as well as the binarized and pseudo-colored nuclei image stack masks. Within the interface, the data cube is navigated by hand and channel visibility and maximum intensity projection thickness can be managed in order to locate nuclei throughout the dataset. Once nuclei are selected by hand, the corresponding nucleus within the Imaris statistics data is identified based on the distance between the clicked location and the array of nuclei centroids exported from Imaris. After identifying the cell type, the GUI collects a variety of information from each selected nucleus. First, it cross-references the position, volume and sphericity from the exported Imaris statistics. Second, it carries out a variety of dilation procedures using 15 distinct dilation amounts pixels $[2,3,4,5,7,10,12,15,20,25,30,35,40,45,50]$. For each dilation, the fluorescence intensity within the perinuclear zone of a given nucleus was collected in the Smi-311, GFAP and VWF channels. The mean, standard deviation and number of pixels are collected for each combination of dilation and channel. Although the size of the perinuclear zone may vary with the nuclear size, this factor is not considered since nuclei volume remains relatively stable and the perinuclear area is stained regardless of variations in nuclear size. By collecting perinuclear data at such a wide variety of radii around the nuclear edge, subsequent optimization practices aim to identify the optimal dilations to maximize sensitivity and specificity of cell-type identification.

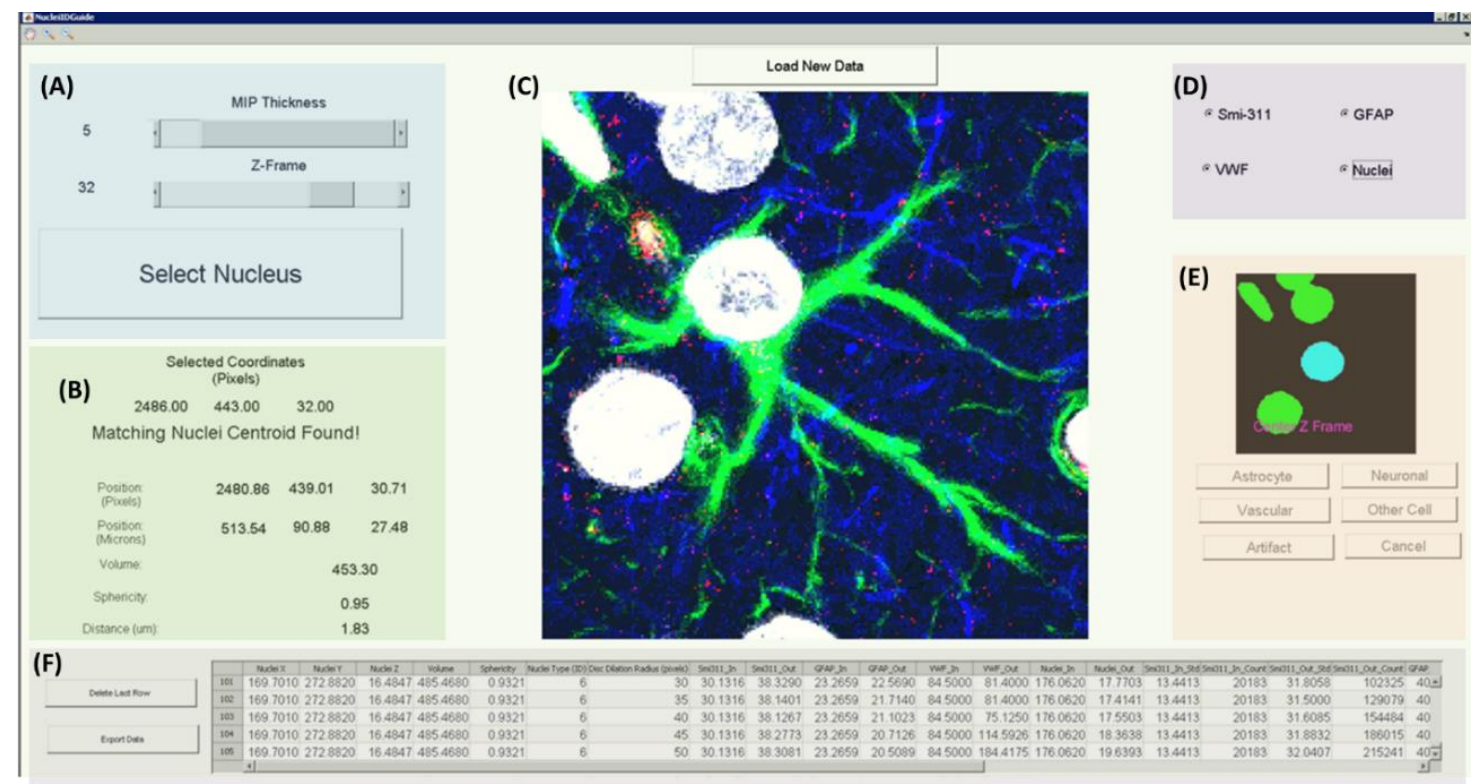

Figure 4.3: Matlab GUI for Nuclei Annotation \& Feature Extraction. To use the GUI, the dataset is first navigated to highlight a suitable nucleus for annotation. Manipulation of the (A) maximum intensity projection and Z-frame using sliders as well as the (D) visibility of the four fluorescently stained channels using radio buttons allows visual confirmation of the cellular origin of the nuclei of interest. After clicking 'Select Nuclei' the user clicks the nuclei of interest. Following this, the closest matching nucleus from the Imaris output is identified and relevant information is presented $(B)$ such as the position of the centroid, volume, sphericity, and the distance of the centroid from the location clicked (to validate accuracy). Following this, the chosen nuclei is (E) highlighted automatically and separated from surrounding nuclei as a Z-stack is panned from 7 frames below the centroid until 7 frames above 
the centroid. This allows clear visualization for validation of nuclei individualization. The same Z-panning is then automatically displayed while the selected nuclei is superimposed onto the remaining fluorescent channels to ensure a correct selection is made. Finally, once completed the user can choose the identity of the selected object. After the selection is made, perinuclear parameters are extracted and collected in $(F)$ for all fifteen binary dilation parameters for the chosen nuclei. Once this entire process has been carried out for each nucleus, data can then be conveniently exported within the GUI to an Excel filetype.

In order to separate cell types once the dilation parameters have been optimized, a 3D support vector machine with a third-order hyperplane was decided as the discrimination methodology. To carry this out, perinuclear fluorescence values for all nuclei are individually normalized in each of the neuronal, astrocytic and vascular channels and each nucleus is plotted in a 3-dimensional array, with one axis per channel. The specific dilation parameter is decided on for each of these three fluorescent channels through optimization experiments and are applied to each of the unknown nuclei. Since all axes are normalized, the hyperplane derived for each decision boundary can be used throughout all data sets.

To optimize dilation parameters, annotated data was independently pooled between both hemispheres of the M1 dataset and both hemispheres of the S1BF dataset, with the goal of using one as a training set and the other as a testing set. First, M1 data was used for training and S1BF was used for testing in order to assess the influence of nuclei pixel dilation on sensitivity and specificity. Then, the training and testing sets were reversed, and the process was carried out again. The two sets of results were averaged to identify the overall effect of dilation quantity on sensitivity and specificity. Parameter optimization utilizing the previously described list of dilation amounts to maximize sensitivity and specificity. Vascular channel pixel dilation was assessed separately from neuronal and astrocytic channel pixel dilation, which were grouped together. Analysis was performed in this way since vascular-type cells (i.e. endothelial cells, pericytes) would not have typical perinuclear staining since the vascular stain was lumenal in nature. On the other hand, neuronal and astrocytic cells were expected to have typical perinuclear staining since they are distinct cells with a soma surrounding the nucleus. Once the optimal per-channel dilation parameters were chosen through this methodology, all data was pooled together to carry out a typical 70\%/30\% randomized training/testing analysis. The process of randomization into training and testing sets and subsequent assessment of sensitivity and specificity was carried out 1000 times to understand overall algorithm ability and variability.

The final outcome of this process is the automated classification of all nuclei in a dataset, regardless of its size. Once completed, the binary masks of chosen nuclei (such as Astrocytic nuclei) are 
modified to have a pixel value slightly above the dataset saturation value, after which they are re-added back into the fluorescent channel of their specific cell type. This process results in an image stack where nuclei for the cell of interest has been superimposed into the channel. As a result of this process and the brighter pixel value of the nuclei, Imaris can then be leveraged to identify each nuclei as a seed point for subsequent volumetric and filament modeling. With the clear identification of each cell's soma, automated cell individualization and morphological extraction through volumetric and filament-based modeling can proceed reliably since cell boundaries in a dense network can be separated using the watershed transform.

The flowchart in Figure 4.4 highlights the sequence of steps carried out in this methodology.

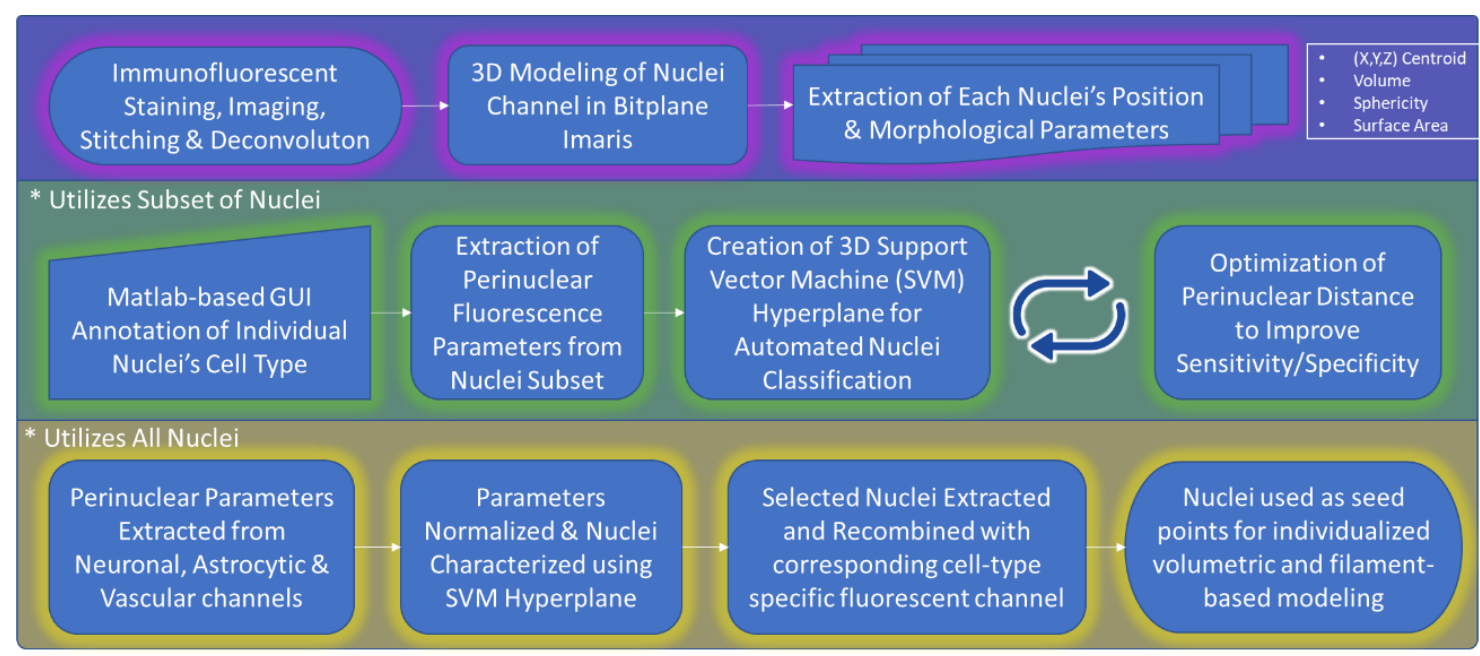

Figure 4.4: Flowchart of Protocol Methodology. The first sequence of steps, highlighted in purple, include the process of raw data collection and $3 D$ modeling of the nuclei channel within Imaris, after which a variety of morphological parameters (centroid, volume, sphericity, surface area) are collected for each individual nucleus object. Following this, the second sequence of steps, highlighted in green, are used to develop the nuclei classification model, using a subset of dataset nuclei. First, nuclei from different slices, depths and functional regions are manually annotated using a GUI, after which Imaris morphological parameters and perinuclear region pixel values from all fluorescent channels are collected for various diameters around each nucleus. An iterative optimization process is then carried out, during which various combinations of perinuclear diameters are used, with each generating a new SVM hyperplane and resultant sensitivity/specificity value. Once the optimal parameters are chosen, a finalized classification hyperplane is created, and the final step, highlighted in yellow, is carried out with all dataset nuclei. The finalized nuclei classification hyperplane from step two is combined with normalized per-nuclei parameters for all dataset nuclei to allow rapid automation of vast quantities of nuclei within the datasets. Following this, nuclei are 3-dimensionally recombined with the corresponding channel of the cell they are assigned to, allowing them to be used as seed points for cell individualization.

\section{Results}

Preliminary optimization required determining the ideal perinuclear distance from the nuclei boundary to maximize sensitivity and specificity of the cell-type determination. The results of this analysis for astrocyte cell-type identification are provided in Table 4.1. Optimal perinuclear distance from the 
astrocytic and neuronal channel is only a few pixels, which matches expectations due to the very thin perinuclear stained zone.

\section{Sensitivity}

\begin{tabular}{|c|c|c|c|c|c|c|c|c|c|c|c|c|c|c|c|c|}
\hline & & \multicolumn{15}{|c|}{ Perinuclear Distance from Nuclei Boundary (Pixels): Astrocyte \& Neuronal Channel } \\
\hline & & 2 & 3 & 4 & 5 & 7 & 10 & 12 & 15 & 20 & 25 & 30 & 35 & 40 & 45 & 50 \\
\hline \multirow{15}{*}{ 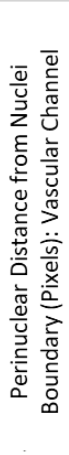 } & 2 & 849 & 953 & 10 & 855 & 788 & 22 & 809 & 52 & 590 & 672 & 694 & 628 & 627 & 667 & 19 \\
\hline & 3 & & & & 66 & & & & & 49 & 718 & 308 & & & 667 & 810 \\
\hline & 4 & 887 & 953 & 317 & 885 & 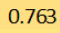 & 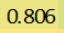 & 0 & & 05 & 732 & 308 & & & & 657 \\
\hline & 5 & 0.912 & 0.955 & 929 & 0.905 & 0.778 & 775 & .764 & 0.780 & 833 & 768 & .778 & 597 & 592 & .604 & .689 \\
\hline & 7 & 914 & .917 & 866 & 849 & 81 & 55 & .764 & 18 & 797 & 829 & 731 & 00 & & 0.729 & .57 \\
\hline & 10 & 0.873 & 7 & 35 & 0.866 & 0.733 & .763 & 6 & 0 & 309 & 784 & 829 & 24 & 83 & 32 & 557 \\
\hline & 12 & 0 & 0.941 & 1 & 0.905 & 0.687 & 0.8 & 0.8 & & 70 & 99 & 02 & 18 & & 41 & 526 \\
\hline & 15 & 0.871 & .865 & 85 & 0.833 & 0.709 & U & & 0.8 & 52 & 55 & 92 & 06 & & & 541 \\
\hline & 20 & 0.910 & 85 & 853 & 0.828 & 0.819 & 76 & 9 & 0 & 00 & 748 & 806 & 06 & 21 & 0.595 & .573 \\
\hline & 25 & 0.937 & 0.9 & 95 & 0.8 & 6 & & & & & 91 & 10 & 76 & & 66 & 621 \\
\hline & 30 & 0.922 & 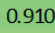 & 85 & 0.8 & 0.8 & 0.83 & 0.77 & 0.8 & 0.690 & 23 & 74 & & & & 0.661 \\
\hline & 35 & 0.908 & 0.899 & 0.885 & 0.855 & 0.856 & & 0.8 & 0.814 & 0.768 & 0.714 & 0.724 & 0.720 & & 0.727 & 0.640 \\
\hline & 40 & 0. & & & & & & & & 0.727 & 0.667 & 70 & 75 & & 0.672 & 0.586 \\
\hline & 45 & 0.868 & 0.895 & 0.856 & 0.851 & 0.842 & 0.8 & & & 0.711 & 0.702 & 0.671 & 0.645 & 52 & 0.696 & .616 \\
\hline & 50 & 0.846 & 0.871 & 0.835 & 0.838 & 0.824 & 0.795 & 0.800 & 0.797 & 0.720 & 0.678 & 0.693 & 0.655 & 0.627 & 0.666 & 0.631 \\
\hline
\end{tabular}

\section{Specificity}

\begin{tabular}{|c|c|c|c|c|c|c|c|c|c|c|c|c|c|c|c|c|}
\hline & & \multicolumn{15}{|c|}{ Perinuclear Distance from Nuclei Boundary (Pixels): Astrocyte \& Neuronal Channel } \\
\hline & & 2 & 3 & 4 & 5 & 7 & 10 & 12 & 15 & 20 & 25 & 30 & 35 & 40 & 45 & 50 \\
\hline \multirow{15}{*}{ 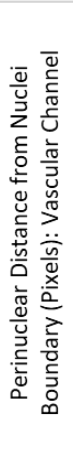 } & 2 & 885 & 943 & 858 & 15 & 683 & 0 & 59 & 0 & & 0.8958 & & 0.8921 & 88 & 48 & \\
\hline & 3 & & & & & & & & & & 61 & & & & 82 & \\
\hline & 4 & 9943 & 0.9779 & 0.9917 & 0.9917 & 0.9683 & 0.9758 & 0.9544 & 0.939 & 0.903 & 0.8823 & & & 0.8557 & 71 & 928 \\
\hline & 5 & 9943 & 9836 & 0.9917 & 0.9758 & 0.9609 & 0.9464 & 0.9389 & 0.926 & 0.8847 & 0.8863 & & 0.8743 & 0.8631 & 43 & 745 \\
\hline & 7 & & & & 0.9 & & & 0.9126 & & & & & & & & \\
\hline & 10 & 0 & 0 & 0. & 0.9758 & 0. & 0. & 0.9084 & 3 & 0. & 72 & 28 & 02 & 28 & & 69 \\
\hline & 12 & & & & 0. & & & 0.915 & 0.8 & & & & & & & \\
\hline & 15 & & & & & & & 0.9132 & 0.8 & & & & & & & \\
\hline & 20 & 0 & 0 & 0.5 & 0.9915 & 0 & 0. & 0.8902 & 0.9 & & 82 & 0.9221 & & 52 & & 79 \\
\hline & 5 & 0 & & 0.9775 & 0.9915 & & 0.9256 & & & & & & & & & 0.89 \\
\hline & 30 & 0.9803 & 0.9858 & 0.9833 & 0.9915 & & & 0.9269 & 0.91 & & & & & & & .919 \\
\hline & 5 & 0.9802 & 0.9915 & 0.9833 & 0.9915 & 0.9603 & 0.9462 & 0.9462 & 0.9338 & 0.8987 & 0.9068 & 0.9189 & 0.8963 & 0.8649 & 0 & 0.8838 \\
\hline & 40 & & 0.9857 & & & & & & & & & & & & & \\
\hline & 45 & 0.9741 & 0.98 & 0.9635 & 0.9754 & & & 0.9394 & 0.9281 & & 45 & 04 & 0.8734 & 0.8644 & 3814 & 0.8504 \\
\hline & 50 & 0.9737 & 0.9798 & 0.9632 & 0.9692 & 0.9754 & 0.9462 & 0.9269 & 0.9218 & 0.9251 & 0.937 & 0.9312 & 0.8748 & 0.8613 & 0.8767 & 0.8561 \\
\hline
\end{tabular}

Table 4.1: Sensitivity/Specificity Optimization of Perinuclear Distance Assessment. Since perinuclear pixel density was collected from 15 unique distances from the nuclei boundary for each channel, it was critical to identify the relationship between the distance chosen and the resulting sensitivity/specificity of the subsequent nuclei identification. To carry out this optimization, neuronal and astrocytic perinuclear distances were kept the same along one axis while the vascular perinuclear distance was separately modified. Neuronal and astrocytic parameters were grouped since nuclei of both cell types have similar thin perinuclear rings, while vascular parameters were separated since the lumen vascular staining does not have the same spatial relationship with endothelial cell nuclei lining vascular structures. To generate this table, data from two cortical columns of one brain slice was used as training data while two cortical columns of a different brain slice was used as testing data. Roles were then reversed, and sensitivity/specificity calculations were run once again. The above tables represent the average values from both between-slice testing routines. Pseudocoloring of cells is performed automatically within Excel to represent the spectrum from favorable (dark green) to unfavorable (dark red) performance.

Based on the results of the sensitivity/specificity testing, the chosen perinuclear distances used were 3 pixels for neuronal/astrocyte channel and 5 pixels for the vascular channel. Based on these parameters, perinuclear staining intensity was calculated for each cell type for the neuronal, astrocytic and vascular channel. Figure 4.5 demonstrates the distinct separation of each cell type along these axes as well as the discrimination hyperplane developed using the support vector machine. 


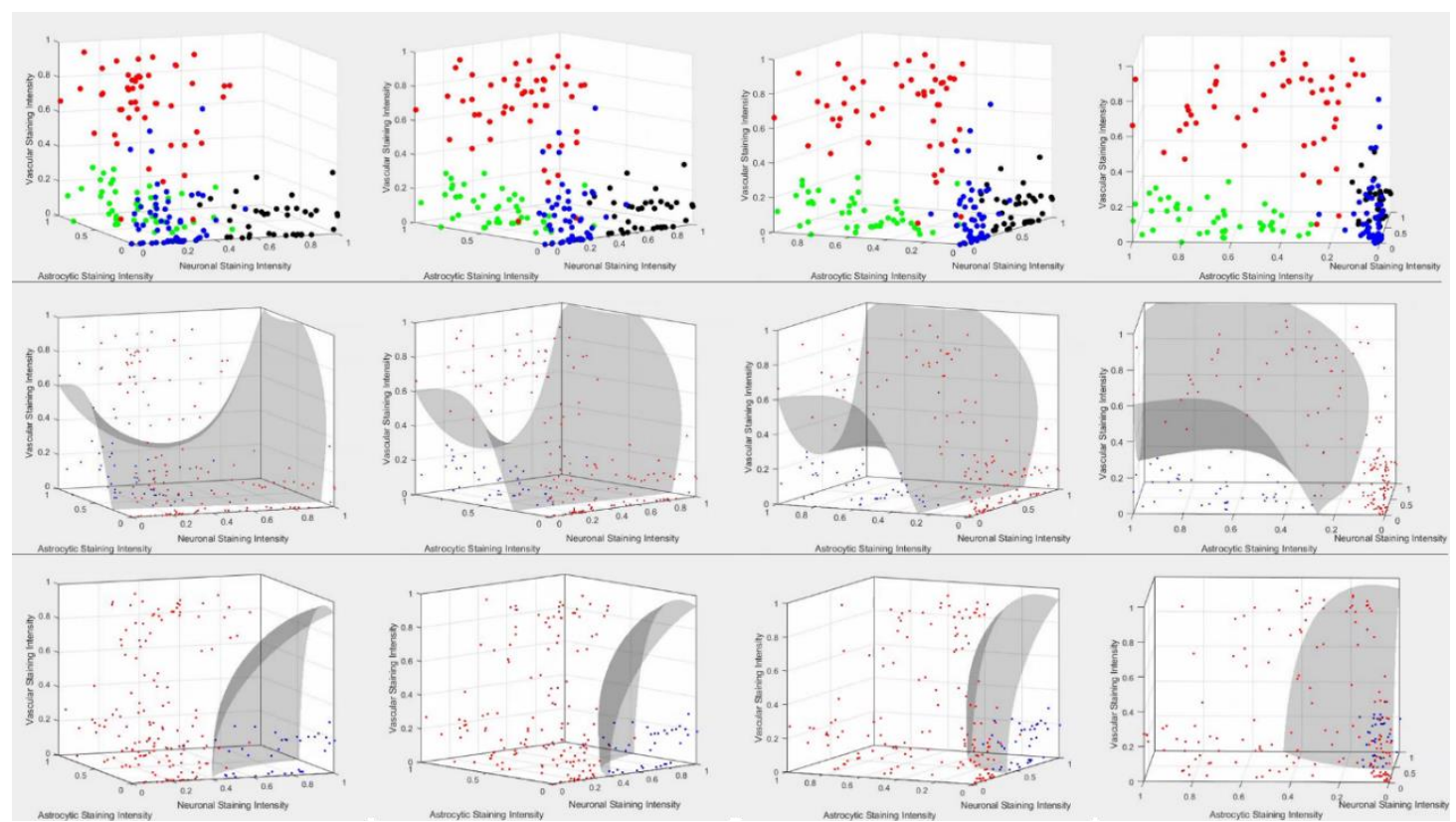

Figure 4.5: Distribution of Nuclei and Hyperplane Development. Images in the first row demonstrate the distribution of Neurons (Black), Astrocytes (Green), Vascular (Red) and Other nuclei (Blue) along the normalized axes of perinuclear staining density in the corresponding fluorescently labeled channels. The second and third row provide the fitted support vector machine hyperplane for astrocytic and neuronal nuclei identification, respectively. In the second and third row, blue dots represent the nuclei of interest while red dots represent all other nuclei, demonstrating the discrimination accuracy of the developed hyperplane. Each column in all rows represents an iterative, synchronized rotation of the $3 D$ plot along the XY plane to aid in visualization.

To provide a final layer of validation as to the power of the discrimination method, the sensitivity and specificity was determined by randomly segregating the annotated data into training (70\%) and testing (30\%) sets, with the procedure repeated 1,000 times and parameters calculated for each cycle. The results of this analysis are shown in Figure 4.7, which demonstrate the ability of this method to accurately identify astrocytic and neuronal cells.

\begin{tabular}{lcc} 
& \multicolumn{2}{c}{ Astrocyte } \\
& Average & Standard Deviation \\
Sensitivity & $97.65 \%$ & $4.46 \%$ \\
Specificity & $98.99 \%$ & $1.49 \%$
\end{tabular}

\begin{tabular}{lcc} 
& \multicolumn{2}{c}{ Neuron } \\
& Average & Standard Deviation \\
Sensitivity & $93.12 \%$ & $6.27 \%$ \\
Specificity & $98.66 \%$ & $1.47 \%$
\end{tabular}

Table 4.2: Result of Randomized Train/Test Groupings. Randomized separation of data into training (70\%) and testing (30\%) groups was conducted 1,000 times in order to identify average and standard deviation of resultant sensitivity and specificity metrics.

Following recombination of chosen nuclei within their corresponding fluorescent channel, individualization of cells in a dense network become substantially easier and no longer require manual 
processing steps. The three astrocytes shown in Figure 4.6 would typically be grouped as one object due to the contact between individual cell end-feet, but with the aid of overlaid nuclei seed points, it becomes simple for the watershed algorithm to identify separation points between these adjacent nuclei. Subsequent analysis based on surface or filamentous rendering can then be carried out in an automated manner, substantially simplifying morphological analysis over large regions of tissue.
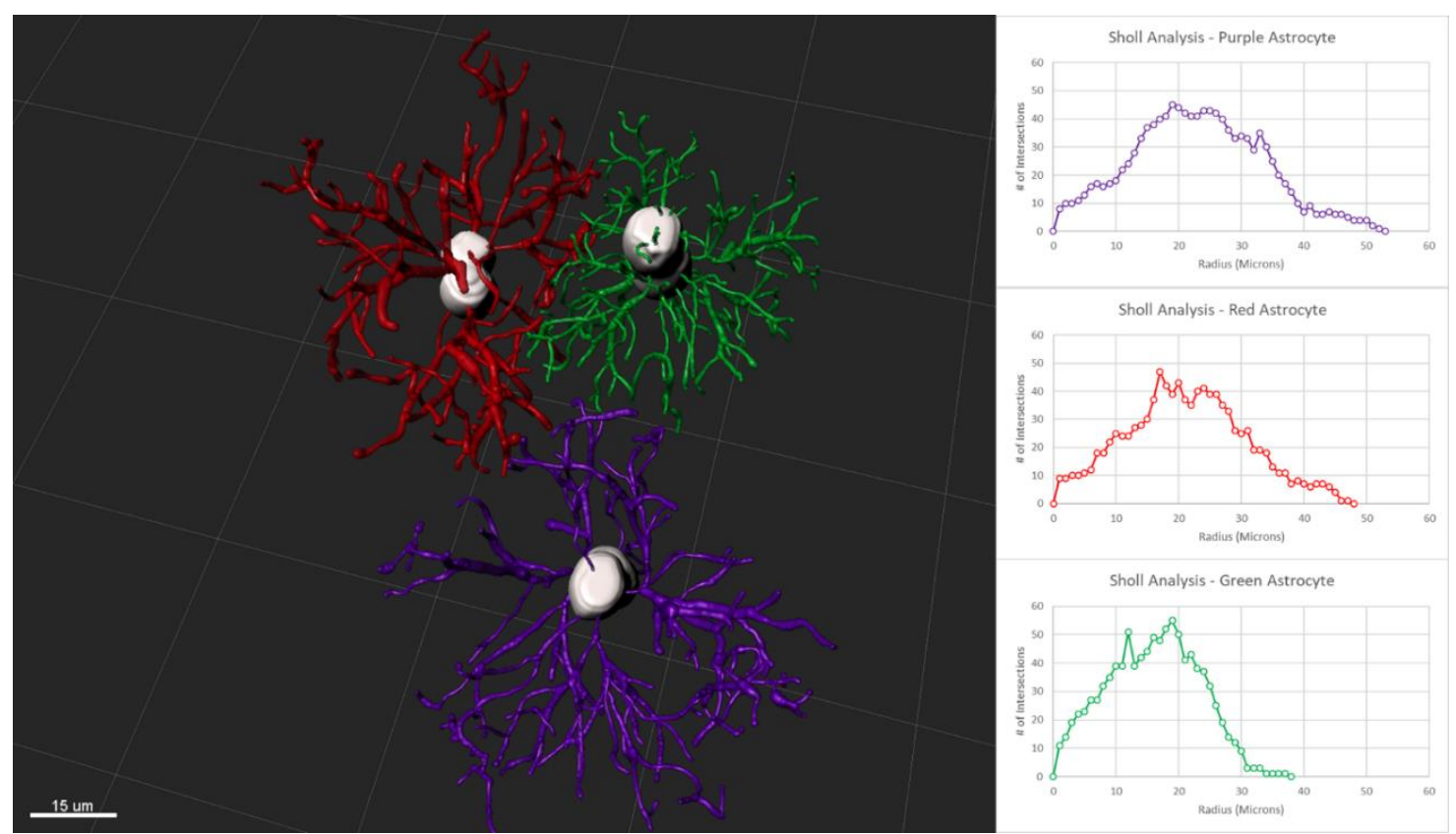

Figure 4.6: Astrocyte Individualization and Parameter Extraction. With the aid of nuclei sorting, astrocyte-specific nuclei are overlaid onto the Astrocyte channel and can be subsequently used as seed points within modeling software such as Bitplane Imaris for cell individualization and cell-specific morphological feature extraction.

\section{Discussion}

Cell type-specific nuclei assignment based on perinuclear fluorescent values emerges as a powerful tool for individualizing cells within a dense network. Furthermore, for cell types that have no distinct nuclear marker, this strategy serves as a blueprint for automating cell identification. In the complex neuropil, resolving this problem has previously required laborious manual tracing and dye-filling. Through this methodology, it is possible to instead utilize semi-automated 3D modeling and morphological analysis capabilities of modern modeling toolboxes to rapidly collect valuable cell-specific data.

Previous attempts to distinguish and individualize these cell types have been carried out in a few different ways. Through assessment of cytological features, one group has attempted identification of 
neuronal, glia and vascular endothelial cells using a decision-making tree which examines the shape of the nucleus, presence of cytoplasm and the distribution of nuclear heterochromatin (García-Cabezas et al., 2016). While an effective method, this technique required many trained raters and the underlying distinction characteristics were more qualitative than quantitative in nature. This method was applied to fluorescent confocal data in a recent study, with the quantitative parameters of texture, intensity and volume used to distinguish neuronal and glial nuclei (Lin et al., 2007) through a similar train/test routine as proposed in this manuscript. The use of perinuclear fluorescence proposed here may provide a more reliable process due to the specificity of immunofluorescently stained cell-specific markers as an identifying feature over parameters such as nuclear intensity or texture.

Prior to deciding on the parameters for separation, various nuclei morphological properties were assessed for their separation ability. Specifically, nuclei volume, sphericity and surface area were evaluated with the annotated data to determine if they were sufficient for clustering the relevant classes of nuclei. None of these parameters demonstrated a clear ability to segregate cell-types of interest, and hence remained unused. Furthermore, it was realized that optimal classification accuracy required assessment of all three relevant staining channels. Assessment of the vascular channel was critical to separate the very similar presentation of astrocytic cells and astrocytic cuffing around vascular components. Additionally, inclusion of the neuronal staining channel helped define the clear cut-off of perinuclear astrocytic staining intensity that reflected an astrocytic vs non-astrocytic cell type.

Once nucleus classification is complete, this protocol provides the critical functionality of aiding morphological characterization. Astrocyte morphology is difficult to study due to their inherent connection to nearly all other surrounding structures in the brain in their supporting role (Mohammed et al., 2018). When attempting to volumetrically render the astrocyte channel, all astrocytes become conjoined into one massive object due to their interconnected structure, and only with a clear definition of each cell's individual soma can cell separation techniques accurately identify appropriate separation points between adjacent cells. The conjoining of adjacent astrocytes is partially due to resolution limitations during imaging and partially due to intrinsic connectivity between adjacent astrocytes. Cell individualization and identification of the soma in an automated fashion allows filament tracing methodologies to accurately 
reconstruct architecture of astrocytic arbors without the need for laborious manual intervention or individual cell dye filling.

When carrying out this protocol, it is critical to ensure that all fluorescent channels are optimally aligned. This is more reliable when the multichannel fluorescent acquisition is collected in a single cycle which means that for each $\mathrm{X}, \mathrm{Y}, \mathrm{Z}$ position all fluorescent channels are collected at the same time before moving to a new position. When attempting to implement this strategy using a collection of previouslyacquired cortical columns, these issues became apparent since of the two microscopes utilized, the first (Olympus FV1000) could only collect up to three channels per cortical column scan (and thus went through two full scan cycles) while the second (Nikon $\mathrm{C} 2+$ ) could be programmed to run the entire cortical column scan in one cycle. As a result of this, only the data from the second microscope could be easily utilized since data from the first microscope had spatially-varying offset between the scans due to independent stitching processes. Error of even a few pixels throughout the dataset is sufficient to prevent optimal application of this algorithm.

Despite the clear advantages of this methodology, a few important limitations exist which must be carefully understood. First, this protocol requires multichannel fluorescent data in order to more reliably distinguish the cell type of interest from others in the region. In the case of astrocytes, this became especially important since the discrimination algorithm could easily mistake nuclei within vascular components due to the astrocytic cuffing that exists in close proximity. Depending on the cell type that researchers need to identify, it is important to consider the cell types most proximal to determine the appropriate targets for other fluorescent channels. Another important limitation to consider was realized in attempts to discriminate neuronal nuclei in addition to astrocytic nuclei. Neuronal dendrites are densely interwoven throughout the cortex, with fine processes from many neurons closely weaving a path around nearly all nuclei present in the dataset. As a result of this, nuclei identification becomes fundamentally less sensitive due to a higher 'background' neuronal-channel signal that surrounds nuclei of any type.

Furthermore, subsequent neuron individualization - even with correctly identified neuronal nuclei - suffers from the same challenges. While the distinct domains of adjacent astrocytes are ideal for subsequent individualization of astrocytes, the interwoven nature of numerous neurons with frequently sub-micron spacing between fine filaments makes individualization of neurons without manual tracing or dye-filling 
nearly impossible. Hence, this methodology is clearly ideal for cells with typically distinct 3 -dimensional domains within interconnected networks. 


\section{Chapter 5. Conclusion}

This PhD dissertation demonstrates numerous methodological advancements which aim to substantially improve the workflow of cortical immunofluorescent staining, imaging and subsequent quantitative analysis. Each stage of this work brings with it both unique enhancements as well as limitations, but as a result of the developed techniques preliminary analyses of tissue architecture and cellular morphology are completed.

The developed immunofluorescent staining and imaging techniques provide improved strategies for high-resolution fluorescence imaging over large 3-dimensional regions. Software-aided alignment of the cortical slice and stereotaxic atlas enhances confidence of localization of the region of interest at high magnification. Tissue compression during antigen retrieval improves epitope availability at various depths without damaging tissue. Finally, a comprehensive factorial design demonstrates that the extended immunofluorescent staining protocol provides quantitatively superior image quality to more typical staining methods. Despite these advances, the most fundamental limitation of the described staining and imaging technique is that the thorough optimization is highly specific for the four fluorescent labels chosen. Researchers with similar goals but different fluorescent labels may not require the unique aspects of the presented methodology, but the techniques used can still prove useful in a protocol optimization workflow. An additional limitation was the cross-reactivity of the neuronal and vascular antibody pairings, which required sequential instead of simultaneous staining - substantially extending the duration of the staining procedure. VWF as a vascular label is also not ubiquitously expressed in the vascular endothelium, reducing its value in continuous vascular tracing throughout the data cube.

The subsequent task of deconvolving the raw microscopy data leverages a novel, low-cost methodology for assessing the spectral and depth-dependent PSF (point-spread function) of the imaging system through the development of a set of fixed-depth optical phantoms. Following the measurement of the PSF, iterative blind deconvolution reduces the presence of spherical aberration which would substantially harm downstream analyses. The developed technique allows researchers to adopt a workflow that can ensure their data is improved in a depth-specific and wavelength-specific manner. Despite these key advantages, several challenges still exist for this methodology. First, measurements of sufficient PSFs 
in each spectral channel and numerous depths are laborious. Second, the relevance of this methodology grows with the brain slice thickness used, and hence researchers using slice thicknesses below 10 um may not find the technique critical. Third, since only PSFs at discrete depths are measured, outcomes are dependent on the spacing between the discrete depths and the nonlinear change in spherical aberration between them. Finally, since the ground truth remains unknown, the quantitative 'improvement' cannot be reliably measured in experimental data.

Fractional Volume Analysis (FVA) provides a simple method for calculating the density of cellular components in the cortical column in both a depth-dependent and depth-independent manner. Furthermore, the subsequent statistical analyses comparing hemispheres, brains and functional regions provide valuable insight as to the intrinsic variability within and between functional regions of healthy tissue. The analysis carried out is novel and provided evidence that the fractional volume occupied by each cellular component in the motor and somatosensory regions largely does not change significantly along the cortical depth axis of the column, with a few relevant exceptions. As many emerging MRI technologies leverage the fractional volume of major cellular components to distinguish disease states, characterizing region and cortical column depth specific FVA in the normal brain may play a critical role in later disease diagnosis. Limitations of this strategy are predominately due to the nature of FVA, which does not provide any cell-specific information but instead examines aggregate filament density proportional to the volume of the region being assessed. Furthermore, as a volumetric measure, FVA is very susceptible to potential error due to incomplete immunofluorescent staining or imaging aberrations such as spherical aberration which can elongate processes axially. While in this work these errors would not affect comparisons made since all datasets were processed equally, other researchers will need to carefully understand these considerations before implementing FVA into their analysis.

Automated nuclei classification solves an important problem in modern neuroscience - how to maximize the number of cell-specific fluorescent markers used without needing a unique nuclear stain for each type of cell. Furthermore, this novel methodology provides a method for cell-specific nuclei assignment for cells that do not yet have a cell-specific nuclear marker available. A critical downstream application of this tool is the subsequent individualization of highly interconnected cells in the neuropil - a process that for cells such as astrocytes can only be currently done through tedious manual tracing or dye- 
filling. Using this improved method, astrocytes can be morphologically analyzed in an automated fashion. Hence, this methodology provides the first published method for automated astrocyte individualization and morphological analysis using traditional confocal fluorescence imaging techniques. Despite the importance of this method, it is important to recognize that the sensitivity and specificity of the nuclei assignment methodology for astrocytes is extremely high (97.7\%/99.0\%, respectively) but not $100 \%$. Due to this, a degree of manual user review is useful to ensure that all modeled objects are astrocytes. Furthermore, subsequent astrocyte individualization in a highly interconnected network uses individual nuclei as seed points, and thus proper splitting of adjacent cells requires sufficient staining and post-deconvolution quality to ensure accurate separation. Finally, this methodology works best with a multichannel fluorescent dataset and cell types that occupy unique, interconnected domains in order to separate, reducing the number of cell types that are appropriate with this strategy.

Future work utilizing the diverse tools and technologies developed in this dissertation should focus on the analysis of a disease state, such as focal epilepsy. Focal epilepsy will allow hemispheric comparison to better extract disease-state differences in cortical architecture as the disease state will be within only one hemisphere. Additional value can be extracted through comparisons of extracted metrics with those from the normal brain established in this dissertation. Furthermore, it may be valuable to test alternative vascular markers to improve the homogeneity of vascular staining as well as neuronal markers to reduce the need for extended immunofluorescent staining durations. Automated nuclei assignment can also be tested with other relevant cells in the cortical column, such as microglia. Finally, a more comprehensive analysis of astrocyte morphology can be carried out using extracted quantitative metrics, which can be used to cluster astrocytes into morphological sub-types for further evaluation. This dissertation develops a wide variety of novel tools and technologies to augment the existing neuroscience workflow, and thus it can be used to enhance a vast array of different research projects across multiple disciplines. 
References

Adriani, G., Ma, D., Pavesi, A., Kamm, R. D., \& Goh, E. L. K. (2017). A 3D neurovascular microfluidic model consisting of neurons, astrocytes and cerebral endothelial cells as a blood-brain barrier. Lab on a Chip, 17(3), 448-459. https://doi.org/10.1039/C6LC00638H'

Aguet, F., Van De Ville, D., \& Unser, M. (2008). An accurate PSF model with few parameters for axially shift-variant deconvolution. 2008 5th IEEE International Symposium on Biomedical Imaging: From Nano to Macro, 157-160. https://doi.org/10.1109/ISBI.2008.4540956

Alberts, B., Johnson, A., Lewis, J., Raff, M., Roberts, K., \& Walter, P. (2002). Molecular Biology of the Cell (4th Editio). Garland Science. https://doi.org/10.1097/00024382-200209000-00015

Bar El, Y., Kanner, S., Barzilai, A., \& Hanein, Y. (2018). Activity changes in neuron-astrocyte networks in culture under the effect of norepinephrine. PLOS ONE, 13(10), e0203761. https://doi.org/10.1371/journal.pone.0203761

Barbas, H., \& García-Cabezas, M. Á. (2015). Motor cortex layer 4: less is more. Trends in Neurosciences, 38(5), 259-261. https://doi.org/10.1016/j.tins.2015.03.005

Bélanger, M., Allaman, I., \& Magistretti, P. J. (2011). Brain Energy Metabolism: Focus on AstrocyteNeuron Metabolic Cooperation. Cell Metabolism, 14(6), 724-738.

https://doi.org/10.1016/j.cmet.2011.08.016

Belichenko, P. V., Sourander, P., Malmgren, K., Nordborg, C., von Essen, C., Rydenhag, B., Lindström, S., Hedström, A., Uvebrant, P., \& Dahlström, A. (1994). Dendritic morphology in epileptogenic cortex from TRPE patients, revealed by intracellular Lucifer Yellow microinjection and confocal laser scanning microscopy. Epilepsy Research, 18(3), 233-247. https://doi.org/10.1016/09201211(94)90044-2

Ben Hadj, S., Blanc-Feraud, L., Aubert, G., Engler, G., Maalouf, E., Colicchio, B., \& Dieterlen, A. (2013). Blind depth-variant blur removal in confocal microscopy. 2013 IEEE 10th International Symposium on Biomedical Imaging, 165-168. https://doi.org/10.1109/ISBI.2013.6556438

Ben Menachem-Zidon, O., Avital, A., Ben-Menahem, Y., Goshen, I., Kreisel, T., Shmueli, E. M., Segal, M., Ben Hur, T., \& Yirmiya, R. (2011). Astrocytes support hippocampal-dependent memory and long-term potentiation via interleukin-1 signaling. Brain, Behavior, and Immunity, 25(5), 1008-1016. https://doi.org/10.1016/j.bbi.2010.11.007

Beutner, E. H. (2003). The development of immunofluorescence and the immunopathology of the skin. 99109.

Biggs, D. S. C. (2010). 3D Deconvolution Microscopy. Current Protocols in Cytometry, 52(1), 12.19.112.19.20. https://doi.org/10.1002/0471142956.cy1219s52

Biggs, D. S. C., \& Andrews, M. (1997). Acceleration of iterative image restoration algorithms. Applied Optics, 36(8), 1766. https://doi.org/10.1364/AO.36.001766

Bopp, R., Holler-Rickauer, S., Martin, K. A. C., \& Schuhknecht, G. F. P. (2017). An Ultrastructural Study of the Thalamic Input to Layer 4 of Primary Motor and Primary Somatosensory Cortex in the Mouse. The Journal of Neuroscience, 37(9), 2435-2448. https://doi.org/10.1523/JNEUROSCI.2557-16.2017

Boutet de Monvel, J., Le Calvez, S., \& Ulfendahl, M. (2001). Image Restoration for Confocal Microscopy: Improving the Limits of Deconvolution, with Application to the Visualization of the Mammalian 
Hearing Organ. Biophysical Journal, 80(5), 2455-2470. https://doi.org/10.1016/S00063495(01)76214-5

Bria, A., \& Iannello, G. (2012). TeraStitcher - A tool for fast automatic 3D-stitching of teravoxel-sized microscopy images.

Briggman, K. L., \& Denk, W. (2006). Towards neural circuit reconstruction with volume electron microscopy techniques. Current Opinion in Neurobiology, 16(5), 562-570. https://doi.org/10.1016/j.conb.2006.08.010

Brown, C. M., Dalal, R. B., Hebert, B., Digman, M. A., Horwitz, A. R., \& Gratton, E. (2008). Raster image correlation spectroscopy (RICS) for measuring fast protein dynamics and concentrations with a commercial laser scanning confocal microscope. Journal of Microscopy, 229(1), 78-91. https://doi.org/10.1111/j.1365-2818.2007.01871.x

Bushong, E. A., Martone, M. E., Jones, Y. Z., \& Ellisman, M. H. (2002). Protoplasmic Astrocytes in CA1 Stratum Radiatum Occupy Separate Anatomical Domains. The Journal of Neuroscience, 22(1), 183192. https://doi.org/10.1523/JNEUROSCI.22-01-00183.2002

Čapek, M., Janáček, J., \& Kubínová, L. (2006). Methods for compensation of the light attenuation with depth of images captured by a confocal microscope. Microscopy Research and Technique, 69(8), 624-635. https://doi.org/10.1002/jemt.20330

Cerbai, F., Lana, D., Nosi, D., Petkova-kirova, P., Zecchi, S., Brothers, H. M., Wenk, G. L., \& Giovannini, M. G. (2012). The Neuron-Astrocyte-Microglia Triad in Normal Brain Ageing and in a Model of Neuroinflammation in the Rat Hippocampus. 7(9). https://doi.org/10.1371/journal.pone.0045250

Chen, J., Lin, R., Wang, H., Meng, J., Zheng, H., \& Song, L. (2013). Blind-deconvolution opticalresolution photoacoustic microscopy in vivo. Optics Express, 21(6), 7316.

https://doi.org/10.1364/OE.21.007316

Chung, W., Allen, N. J., \& Eroglu, C. (2015). Astrocytes Control Synapse Formation, Function, and Elimination. Cold Spring Harbor Perspectives in Biology, 7(9), a020370. https://doi.org/10.1101/cshperspect.a020370

Cogswell, C., \& Sheppard, C. (2014). Confocal Brightfield Imaging Techniques Using an On-Axis Scanning Optical Microscope. In Confocal Microscopy (Issue May, pp. 213-243).

Cooper, G. (2000). The Cell: A Molecular Approach (2nd Editio). Sinauer Associates.

De Jonge, N., \& Peckys, D. B. (2016). Live Cell Electron Microscopy Is Probably Impossible. ACS Nano, 10(10), 9061-9063. https://doi.org/10.1021/acsnano.6b02809

Deonarain, M., Yahioglu, G., Stamati, I., Pomowski, A., Clarke, J., Edwards, B., Diez-Posada, S., \& Stewart, A. (2018). Small-Format Drug Conjugates: A Viable Alternative to ADCs for Solid Tumours? Antibodies, 7(2), 16. https://doi.org/10.3390/antib7020016

Dobson, P. F., Rocha, M. C., Grady, J. P., Chrysostomou, A., Hipps, D., Watson, S., Greaves, L. C., Deehan, D. J., \& Turnbull, D. M. (2016). Unique quadruple immunofluorescence assay demonstrates mitochondrial respiratory chain dysfunction in osteoblasts of aged and PolgA- -- mice. Scientific Reports, 6(1), 31907. https://doi.org/10.1038/srep31907

Domínguez-Álvaro, M., Montero-Crespo, M., Blazquez-Llorca, L., Insausti, R., DeFelipe, J., \& AlonsoNanclares, L. (2018). Three-dimensional analysis of synapses in the transentorhinal cortex of 
Alzheimer's disease patients. Acta Neuropathologica Communications, 6(1), 20.

https://doi.org/10.1186/s40478-018-0520-6

Eilam, R., Aharoni, R., Arnon, R., \& Malach, R. (2016). Astrocyte morphology is confined by cortical functional boundaries in mammals ranging from mice to human. 1-18. https://doi.org/10.7554/eLife.15915

Ferri, G., Gaudio, R. M., Castello, I. F., Berger, P., \& Giro, G. (1997). Quadruple Immunofluorescence : A Direct Visualization Method. 45(2), 155-158.

Fitzpatrick, A. W. P., Falcon, B., He, S., Murzin, A. G., Murshudov, G., Garringer, H. J., Crowther, R. A., Ghetti, B., Goedert, M., \& Scheres, S. H. W. (2017). Cryo-EM structures of tau filaments from Alzheimer's disease. Nature, 547(7662), 185-190. https://doi.org/10.1038/nature23002

Fulwyler, M., Hanley, Q. S., Schnetter, C., Young, I. T., Jares-Erijman, E. A., Arndt-Jovin, D. J., \& Jovin, T. M. (2005). Selective photoreactions in a programmable array microscope (PAM): Photoinitiated polymerization, photodecaging, and photochromic conversion. Cytometry Part A, 67A(2), 68-75. https://doi.org/10.1002/cyto.a.20174

Gao, R., Asano, S. M., Upadhyayula, S., Pisarev, I., Milkie, D. E., Liu, T., Singh, V., Graves, A., Huynh, G. H., Zhao, Y., Bogovic, J., Colonell, J., Ott, C. M., Zugates, C., Tappan, S., Rodriguez, A., Mosaliganti, K. R., Sheu, S., Pasolli, H. A., ... Betzig, E. (2019). Cortical column and whole-brain imaging with molecular contrast and nanoscale resolution. Science, 363(6424).

https://doi.org/10.1126/science.aau8302

García-Cabezas, M. Á., John, Y. J., Barbas, H., \& Zikopoulos, B. (2016). Distinction of Neurons, Glia and Endothelial Cells in the Cerebral Cortex: An Algorithm Based on Cytological Features. Frontiers in Neuroanatomy, 10(NOV), 1-28. https://doi.org/10.3389/fnana.2016.00107

Gelderblom, M., Weymar, A., Bernreuther, C., Velden, J., Arunachalam, P., Steinbach, K., Orthey, E., Arumugam, T. V, Leypoldt, F., Simova, O., Thom, V., Friese, M. A., Prinz, I., Hölscher, C., Glatzel, M., Korn, T., Gerloff, C., Tolosa, E., \& Magnus, T. (2012). Neutralization of the IL-17 axis diminishes neutrophil invasion and protects from ischemic stroke. Blood, 120(18), 3793-3802. https://doi.org/10.1182/blood-2012-02-412726

Gilani, N., Hildebrand, S., Schueth, A., \& Roebroeck, A. (2019). Monte Carlo Simulation of Diffusion MRI in geometries constructed from two-photon microscopy of human cortical grey matter. BioRxiv, 626945. https://doi.org/https://doi.org/10.1101/626945

Girkin, J. M., \& Carvalho, M. T. (2018). The light-sheet microscopy revolution. Journal of Optics, 20(5), 053002. https://doi.org/10.1088/2040-8986/aab58a

Golgi, C. (1873). Sulla struttura della sostanza grigia del cervello. Gazz Med Ita Lombarda, 33, 244-246.

Gong, H., Zeng, S., Yan, C., Lv, X., Yang, Z., Xu, T., Feng, Z., Ding, W., Qi, X., Li, A., Wu, J., \& Luo, Q. (2013). NeuroImage Continuously tracing brain-wide long-distance axonal projections in mice at a one-micron voxel resolution. NeuroImage, 74, 87-98. https://doi.org/10.1016/j.neuroimage.2013.02.005

Gonzalez-bellido, P. (2012). Labeling and Confocal Imaging of Neurons in Thick Invertebrate Tissue Samples. May 2014. https://doi.org/10.1101/pdb.prot069625

Grabinski, T. M., Kneynsberg, A., Manfredsson, F. P., \& Kanaan, N. M. (2015). A Method for Combining RNAscope In Situ Hybridization with Immunohistochemistry in Thick Free-Floating Brain Sections and Primary Neuronal Cultures. 1-19. https://doi.org/10.1371/journal.pone.0120120 
Gräf, R., Rietdorf, J., \& Zimmermann, T. (2005). Live Cell Spinning Disk Microscopy. In Advances in Biochemical Engineering/Biotechnology (Vol. 95, Issue September 2015, pp. 57-75). https://doi.org/10.1007/b102210

Grünewald, A., Lax, N. Z., Rocha, M. C., Reeve, A. K., Hepplewhite, P. D., A. Rygiel, K., Taylor, R. W., \& Turnbull, D. M. (2014). Quantitative quadruple-label immunofluorescence of mitochondrial and cytoplasmic proteins in single neurons from human midbrain tissue. Journal of Neuroscience Methods, 232, 143-149. https://doi.org/10.1016/j.jneumeth.2014.05.026

Haseleu, J., Anlauf, E., Blaess, S., Endl, E., \& Derouiche, A. (2013). Studying subcellular detail in fixed astrocytes : dissociation of morphologically intact glial cells (DIMIGs ). 7(May), 1-10. https://doi.org/10.3389/fncel.2013.00054

Herculano-Houzel, S., Watson, C., \& Paxinos, G. (2013). Distribution of neurons in functional areas of the mouse cerebral cortex reveals quantitatively different cortical zones. Frontiers in Neuroanatomy, 7(OCT), 1-14. https://doi.org/10.3389/fnana.2013.00035

Hernández Candia, C. N., \& Gutiérrez-Medina, B. (2014). Direct Imaging of Phase Objects Enables Conventional Deconvolution in Bright Field Light Microscopy. PLoS ONE, 9(2), e89106. https://doi.org/10.1371/journal.pone.0089106

Hillman, E. M. C., Voleti, V., Li, W., \& Yu, H. (2019). Light-Sheet Microscopy in Neuroscience. Annual Review of Neuroscience, 42(1), 295-313. https://doi.org/10.1146/annurev-neuro-070918-050357

Hirano, A., Kawanami, T., \& Llena, J. F. (1994). Electron microscopy of the blood-brain barrier in disease. Microscopy Research and Technique, 27(6), 543-556. https://doi.org/10.1002/jemt.1070270609

Hoffpauir, B. K., Pope, B. A., \& Spirou, G. A. (2007). Serial sectioning and electron microscopy of large tissue volumes for 3D analysis and reconstruction: a case study of the calyx of Held. Nature Protocols, 2(1), 9-22. https://doi.org/10.1038/nprot.2007.9

Holmes, T. J., Bhattacharyya, S., Cooper, J. A., Hanzel, D., Krishnamurthi, V., Lin, W., Roysam, B., Szarowski, D. H., \& Turner, J. N. (1995). Light Microscopic Images Reconstructed by Maximum Likelihood Deconvolution. In Handbook of Biological Confocal Microscopy (pp. 389-402). Springer US. https://doi.org/10.1007/978-1-4757-5348-6_24

Jiang, M., \& Wang, G. (2003). Development of blind image deconvolution and its applications. Journal of $X$-Ray Science and Technology, 11(1), 13-19.

Kälin, S., Heppner, F. L., Bechmann, I., Prinz, M., Tschöp, M. H., \& Yi, C. (2015). Hypothalamic innate immune reaction in obesity. Nature Publishing Group, 11(June), 339-351. https://doi.org/10.1038/nrendo.2015.48

Kaplan, E. S., Ramos-Laguna, K. A., Mihalas, A. B., Daza, R. A. M., \& Hevner, R. F. (2017). Neocortical Sox9+ radial glia generate glutamatergic neurons for all layers, but lack discernible evidence of early laminar fate restriction. Neural Development, 12(1), 14. https://doi.org/10.1186/s13064-017-0091-4

Karbowski, J. (2015). Cortical Composition Hierarchy Driven by Spine Proportion Economical Maximization or Wire Volume Minimization. PLOS Computational Biology, 11(10), e1004532. https://doi.org/10.1371/journal.pcbi.1004532

Keller, D., Erö, C., \& Markram, H. (2018). Cell Densities in the Mouse Brain: A Systematic Review. Frontiers in Neuroanatomy, 12(October). https://doi.org/10.3389/fnana.2018.00083 
Khakh, B. S., \& Deneen, B. (2019). The Emerging Nature of Astrocyte Diversity. Annual Review of Neuroscience, 42(1), 187-207. https://doi.org/10.1146/annurev-neuro-070918-050443

Khoradmehr, A., Sc, M., Mazaheri, F., Sc, M., Anvari, M., Ph, D., Tamadon, A., \& Ph, D. (2019). A Simple Technique for Three-Dimensional Imaging and Segmentation of Brain Vasculature Using Fast Freeof-Acrylamide Clearing Tissue in Murine. 21(1), 49-56. https://doi.org/10.22074/cellj.2019.5684.Introduction

Kimbrough, I. F., Robel, S., Roberson, E. D., \& Sontheimer, H. (2015). Vascular amyloidosis impairs the gliovascular unit in a mouse model of Alzheimer's disease. 3716-3733. https://doi.org/10.1093/brain/awv327

Kobat, D., Horton, N. G., \& Xu, C. (2011). In vivo two-photon microscopy to 1.6-mm depth in mouse cortex. Journal of Biomedical Optics, 16(10), 106014. https://doi.org/10.1117/1.3646209

Koho, S., Fazeli, E., Eriksson, J. E., \& Hänninen, P. E. (2016). Image Quality Ranking Method for Microscopy. Nature Publishing Group, 1-15. https://doi.org/10.1038/srep28962

Konig, K. (2000). Multiphoton microscopy in life sciences. Journal of Microscopy, 200(2), 83-104. https://doi.org/10.1046/j.1365-2818.2000.00738.x

Lai, H. M., Ng, W.-L., Gentleman, S. M., \& Wu, W. (2017). Chemical Probes for Visualizing Intact Animal and Human Brain Tissue. Cell Chemical Biology, 24(6), 659-672. https://doi.org/10.1016/j.chembiol.2017.05.015

Lanjakornsiripan, D., Pior, B.-J., Kawaguchi, D., Furutachi, S., Tahara, T., Katsuyama, Y., Suzuki, Y., Fukazawa, Y., \& Gotoh, Y. (2018). Layer-specific morphological and molecular differences in neocortical astrocytes and their dependence on neuronal layers. Nature Communications, 9(1), 1623. https://doi.org/10.1038/s41467-018-03940-3

Larson, A. M. (2011). Multiphoton microscopy. Nature Photonics, 5(1), 1-1. https://doi.org/10.1038/nphoton.an.2010.2

Lauer, S. M., Schneeweiß, U., Brecht, M., \& Ray, S. (2018). Visualization of Cortical Modules in Flattened Mammalian Cortices. January, 1-9. https://doi.org/10.3791/56992

Lavialle, M., Aumann, G., Anlauf, E., Pröls, F., Arpin, M., \& Derouiche, A. (2011). Structural plasticity of perisynaptic astrocyte processes involves ezrin and metabotropic glutamate receptors. 108(31), 12915-12919. https://doi.org/10.1073/pnas.1100957108

Lee, K. M., Chiu, K. B., Renner, N. A., Sansing, H. A., Didier, P. J., \& MacLean, A. G. (2014). Form follows function: astrocyte morphology and immune dysfunction in SIV neuroAIDS. Journal of NeuroVirology, 20(5), 474-484. https://doi.org/10.1007/s13365-014-0267-1

Lee, S., Han, S., Salama, P., Dunn, K. W., \& Delp, E. J. (2019). Three Dimensional Blind Image Deconvolution for Fluorescence Microscopy using Generative Adversarial Networks. 2019 IEEE 16th International Symposium on Biomedical Imaging (ISBI 2019), 538-542. https://doi.org/10.1109/ISBI.2019.8759250

Lefort, S., Tomm, C., Floyd Sarria, J.-C., \& Petersen, C. C. H. (2009). The Excitatory Neuronal Network of the C2 Barrel Column in Mouse Primary Somatosensory Cortex. Neuron, 61(2), 301-316. https://doi.org/10.1016/j.neuron.2008.12.020 
Lehre, K. P., \& Rusakov, D. A. (2002). Asymmetry of Glia near Central Synapses Favors Presynaptically Directed Glutamate Escape. Biophysical Journal, 83(1), 125-134. https://doi.org/10.1016/S00063495(02)75154-0

Lin, G., Chawla, M. K., Olson, K., Barnes, C. A., Guzowski, J. F., Bjornsson, C., Shain, W., \& Roysam, B. (2007). A multi-model approach to simultaneous segmentation and classification of heterogeneous populations of cell nuclei in 3D confocal microscope images. Cytometry Part A, 71A(9), 724-736. https://doi.org/10.1002/cyto.a.20430

Lu, M., Banetta, L., Young, L. J., Smith, E. J., Bates, G. P., Zaccone, A., Kaminski Schierle, G. S., Tunnacliffe, A., \& Kaminski, C. F. (2019). Live-cell super-resolution microscopy reveals a primary role for diffusion in polyglutamine-driven aggresome assembly. Journal of Biological Chemistry, 294(1), 257-268. https://doi.org/10.1074/jbc.RA118.003500

Maalouf, E., Colicchio, B., \& Dieterlen, A. (2011). Fluorescence microscopy three-dimensional depth variant point spread function interpolation using Zernike moments. Journal of the Optical Society of America A, 28(9), 1864. https://doi.org/10.1364/JOSAA.28.001864

Mahou, P., Vermot, J., Beaurepaire, E., \& Supatto, W. (2014). Multicolor two-photon light-sheet microscopy. Nature Methods, 11(6), 600-601. https://doi.org/10.1038/nmeth.2963

Mahou, P., Zimmerley, M., Loulier, K., Matho, K. S., Labroille, G., Morin, X., Supatto, W., Livet, J., Débarre, D., \& Beaurepaire, E. (2012). Multicolor two-photon tissue imaging by wavelength mixing. 9(8). https://doi.org/10.1038/nmeth.2098

McCaslin, A. F. H., Chen, B. R., Radosevich, A. J., Cauli, B., \& Hillman, E. M. C. (2011). in vivo 3D Morphology of Astrocyte-Vasculature Interactions in the Somatosensory Cortex: Implications for Neurovascular Coupling. Journal of Cerebral Blood Flow \& Metabolism, 31(3), 795-806. https://doi.org/10.1038/jcbfm.2010.204

Medvedev, N., Popov, V., Henneberger, C., Kraev, I., Rusakov, D. A., \& Stewart, M. G. (2014). Glia selectively approach synapses on thin dendritic spines. Philosophical Transactions of the Royal Society B: Biological Sciences, 369(1654), 20140047. https://doi.org/10.1098/rstb.2014.0047

Meyer, H. S., Schwarz, D., Wimmer, V. C., Schmitt, A. C., Kerr, J. N. D., Sakmann, B., \& Helmstaedter, M. (2011). Inhibitory interneurons in a cortical column form hot zones of inhibition in layers 2 and 5A. Proceedings of the National Academy of Sciences, 108(40), 16807-16812. https://doi.org/10.1073/pnas.1113648108

Meyer, K., Ostrenko, O., Bourantas, G., Morales-Navarrete, H., Porat-Shliom, N., Segovia-Miranda, F., Nonaka, H., Ghaemi, A., Verbavatz, J.-M., Brusch, L., Sbalzarini, I., Kalaidzidis, Y., Weigert, R., \& Zerial, M. (2017). A Predictive 3D Multi-Scale Model of Biliary Fluid Dynamics in the Liver Lobule. Cell Systems, 4(3), 277-290.e9. https://doi.org/10.1016/j.cels.2017.02.008

Mlodzianoski, M. J., Cheng-Hathaway, P. J., Bemiller, S. M., McCray, T. J., Liu, S., Miller, D. A., Lamb, B. T., Landreth, G. E., \& Huang, F. (2018). Active PSF shaping and adaptive optics enable volumetric localization microscopy through brain sections. Nature Methods, 15(8), 583-586. https://doi.org/10.1038/s41592-018-0053-8

Moerner, W. E. W. E. (2015). Single-Molecule Spectroscopy, Imaging, and Photocontrol: Foundations for Super-Resolution Microscopy (Nobel Lecture). Angewandte Chemie International Edition, 54(28), 8067-8093. https://doi.org/10.1002/anie.201501949 
Mohammed, H., Al-Awami, A. K., Beyer, J., Cali, C., Magistretti, P., Pfister, H., \& Hadwiger, M. (2018). Abstractocyte: A Visual Tool for Exploring Nanoscale Astroglial Cells. IEEE Transactions on Visualization and Computer Graphics, 24(1), 853-861. https://doi.org/10.1109/TVCG.2017.2744278

Moye, S. L., Diaz-Castro, B., Gangwani, M. R., \& Khakh, B. S. (2019). Visualizing Astrocyte Morphology Using Lucifer Yellow Iontophoresis. Journal of Visualized Experiments, 151, e60225. https://doi.org/10.3791/60225

Nakase, T., Fushiki, S., \& Naus, C. C. G. (2003). Astrocytic Gap Junctions Composed of Connexin 43 Reduce Apoptotic Neuronal Damage in Cerebral Ischemia. Stroke, 34(8), 1987-1993. https://doi.org/10.1161/01.STR.0000079814.72027.34

Nelson, M., Ledoux, J., Taylor, M., Bonev, A., Hannah, R., Solodushko, V., Shui, B., Tallini, Y., \& Kotlikoff, M. (2010). Spinning Disk Confocal Microscopy of Calcium Signalling in Blood Vessel Walls. Microscopy and Analysis (Americas Ed.), 24(2), 5-8. http://www.ncbi.nlm.nih.gov/pubmed/22506097\%0Ahttp://www.pubmedcentral.nih.gov/articlerender .fcgi?artid=PMC3324844

Oberheim, N. A., Goldman, S. A., \& Nedergaard, M. (2012). Heterogeneity of Astrocytic Form and Function (R. Milner (ed.); Vol. 814, pp. 23-45). Humana Press. https://doi.org/10.1007/978-1-61779452-0_3

Ogata, K., \& Kosaka, T. (2002). Structural and quantitative analysis of astrocytes in the mouse hippocampus. Neuroscience, 113(1), 221-233. https://doi.org/10.1016/S0306-4522(02)00041-6

Ohadi, D., \& Rangamani, P. (2019). Geometric Control of Frequency Modulation of cAMP Oscillations due to Calcium in Dendritic Spines. Biophysical Journal, 117(10), 1981-1994. https://doi.org/10.1016/j.bpj.2019.10.004

Pankajakshan, P., Blanc-Feraud, L., Kam, Z., \& Zerubia, J. (2009). Point-Spread Function retrieval for fluorescence microscopy. 2009 IEEE International Symposium on Biomedical Imaging: From Nano to Macro, 1095-1098. https://doi.org/10.1109/ISBI.2009.5193247

Park, H.-J., Lee, K., Park, E. S., Oh, S., Yan, R., Zhang, J., Beach, T. G., Adler, C. H., Voronkov, M., Braithwaite, S. P., Stock, J. B., \& Mouradian, M. M. (2016). Dysregulation of protein phosphatase $2 \mathrm{~A}$ in parkinson disease and dementia with lewy bodies. Annals of Clinical and Translational Neurology, 3(10), 769-780. https://doi.org/10.1002/acn3.337

Park, S., Kang, W., Kwon, Y.-D., Shim, J., Kim, S., Kaang, B.-K., \& Hohng, S. (2018). Superresolution fluorescence microscopy for 3D reconstruction of thick samples. Molecular Brain, 11(1), 17. https://doi.org/10.1186/s13041-018-0361-z

Pathak, A. P., Schmainda, K. M., Ward, B. D., Linderman, J. R., Rebro, K. J., \& Greene, A. S. (2001). MRderived cerebral blood volume maps: Issues regarding histological validation and assessment of tumor angiogenesis. Magnetic Resonance in Medicine, 46(4), 735-747. https://doi.org/10.1002/mrm.1252

Pawley, J. (2006). Handbook Of Biological Confocal Microscopy (J. B. Pawley (ed.)). Springer US. https://doi.org/10.1007/978-0-387-45524-2

Paxinos, G., \& Watson, C. (2007). The Rat Brain in Stereotaxic Coordinates. In Elsevier (6th Editio). Academic Press. https://doi.org/10.1354/vp.43-1-86-a

Pessoa, L. (2014). Understanding brain networks and brain organization. Physics of Life Reviews, 11(3), 400-435. https://doi.org/10.1016/j.plrev.2014.03.005 
Petroff, O. A. C., Pan, J. W., \& Rothman, D. L. (2002). Magnetic Resonance Spectroscopic Studies of Neurotransmitters and Energy Metabolism in Epilepsy. Epilepsia, 43(SUPPL. 1), 40-50. https://doi.org/10.1046/j.1528-1157.2002.043s1040.x

Power, R. M., \& Huisken, J. (2017). A guide to light-sheet fluorescence microscopy for multiscale imaging. Nature Methods, 14(4), 360-373. https://doi.org/10.1038/nmeth.4224

Qin, L., Liu, X., Liu, S., Liu, Y., Yang, Y., Yang, H., Chen, Y., \& Chen, L. (2017). Differentially expressed proteins underlying childhood cortical dysplasia with epilepsy identified by iTRAQ proteomic profiling. PLOS ONE, 12(2), e0172214. https://doi.org/10.1371/journal.pone.0172214

R\&D Systems. (2017). IHC / ICC Protocol Guide. R\&D Systems. https://resources.rndsystems.com/images/site/rnd-systems-ihc-protocol-br.pdf

Reeve, A. K., Grady, J. P., Cosgrave, E. M., Bennison, E., Chen, C., Hepplewhite, P. D., \& Morris, C. M. (2018). Mitochondrial dysfunction within the synapses of substantia nigra neurons in Parkinson ' $\mathrm{s}$ disease. NpjParkinson's Disease, July 2017, 1-10. https://doi.org/10.1038/s41531-018-0044-6

Ritter, P., Schirner, M., Mcintosh, A. R., \& Jirsa, V. K. (2013). The Virtual Brain Integrates Computational Modeling. 3(2). https://doi.org/10.1089/brain.2012.0120

Rizzolatti, G., \& Luppino, G. (2001). The cortical motor system. Neuron, 31(6), 889-901. https://doi.org/10.1016/s0896-6273(01)00423-8

Robel, S., Buckingham, S. C., Boni, J. L., Campbell, S. L., Danbolt, N. C., Riedemann, T., Sutor, B., \& Sontheimer, H. (2015). Reactive Astrogliosis Causes the Development of Spontaneous Seizures. Journal of Neuroscience, 35(8), 3330-3345. https://doi.org/10.1523/JNEUROSCI.1574-14.2015

Rocha, M. C., Grady, J. P., Grünewald, A., Vincent, A., Dobson, P. F., Taylor, R. W., Turnbull, D. M., \& Rygiel, K. A. (2015). A novel immunofluorescent assay to investigate oxidative phosphorylation deficiency in mitochondrial myopathy: understanding mechanisms and improving diagnosis. Scientific Reports, 5(1), 15037. https://doi.org/10.1038/srep15037

Samborska, V., Gordleeva, S., Ullner, E., Lebedeva, A., Kazantsev, V., Ivanchenko, M., \& Zaikin, A. (2016). Mammalian Brain As a Network of Networks. Opera Medica \& Physiologica, 2(1). https://doi.org/https://doi.org/10.20388/omp2016.001.0024

Santi, P. A. (2011). Light Sheet Fluorescence Microscopy. Journal of Histochemistry \& Cytochemistry, 59(2), 129-138. https://doi.org/10.1369/0022155410394857

Saunders, N. R., Dziegielewska, K. M., Møllgård, K., \& Habgood, M. D. (2015). Markers for blood-brain barrier integrity: how appropriate is Evans blue in the twenty-first century and what are the alternatives? Frontiers in Neuroscience, 9(OCT), 1-16. https://doi.org/10.3389/fnins.2015.00385

Savtchenko, L. P., Bard, L., Jensen, T. P., Reynolds, J. P., Kraev, I., Medvedev, N., Stewart, M. G., Henneberger, C., \& Rusakov, D. A. (2018). Disentangling astroglial physiology with a realistic cell model in silico. Nature Communications, 9(1), 3554. https://doi.org/10.1038/s41467-018-05896-w

Sawada, K., Kawakami, R., Shigemoto, R., \& Nemoto, T. (2018). Super-resolution structural analysis of dendritic spines using three-dimensional structured illumination microscopy in cleared mouse brain slices. European Journal of Neuroscience, 47(9), 1033-1042. https://doi.org/10.1111/ejn.13901 
Scalia, C. R., Boi, G., Bolognesi, M. M., Riva, L., Manzoni, M., Desmedt, L., Bosisio, F. M., Ronchi, S., Leone, B. E., \& Cattoretti, G. (2017). Antigen Masking During Fixation and Embedding, Dissected. 65(1). https://doi.org/10.1369/0022155416673995

Schindelin, J., Arganda-Carreras, I., Frise, E., Kaynig, V., Longair, M., Pietzsch, T., Preibisch, S., Rueden, C., Saalfeld, S., Schmid, B., Tinevez, J., White, D. J., Hartenstein, V., Eliceiri, K., Tomancak, P., \& Cardona, A. (2012). Fiji: an open-source platform for biological-image analysis. Nature Methods, 9(7), 676-682. https://doi.org/10.1038/nmeth.2019

Schiweck, J., Eickholt, B. J., \& Murk, K. (2018). Important shapeshifter: Mechanisms allowing astrocytes to respond to the changing nervous system during development, injury and disease. Frontiers in Cellular Neuroscience, 12(August), 1-17. https://doi.org/10.3389/fncel.2018.00261

Schneider, C. A., Rasband, W. S., \& Eliceiri, K. W. (2012). NIH Image to ImageJ: 25 years of image analysis. Nature Methods, 9(7), 671-675. https://doi.org/10.1038/nmeth.2089

Shajkofci, A., \& Liebling, M. (2018). Semi-Blind Spatially-Variant Deconvolution in Optical Microscopy with Local Point Spread Function Estimation by Use of Convolutional Neural Networks. 2018 25th IEEE International Conference on Image Processing (ICIP), 3818-3822. https://doi.org/10.1109/ICIP.2018.8451736

Shioya, M., Obayashi, S., Tabunoki, H., Arima, K., Saito, Y., Ishida, T., \& Satoh, J. (2010). Aberrant microRNA expression in the brains of neurodegenerative diseases: miR-29a decreased in Alzheimer disease brains targets neurone navigator 3. Neuropathology and Applied Neurobiology, 36(4), 320330. https://doi.org/10.1111/j.1365-2990.2010.01076.x

Sigl-Glöckner, J., \& Brecht, M. (2017). Polyploidy and the Cellular and Areal Diversity of Rat Cortical Layer 5 Pyramidal Neurons. Cell Reports, 20(11), 2575-2583. https://doi.org/10.1016/j.celrep.2017.08.069

Strasser, F., Offterdinger, M., Piestun, R., \& Jesacher, A. (2019). Spectral image scanning microscopy. Biomedical Optics Express, 10(5), 2513. https://doi.org/10.1364/BOE.10.002513

Sun, J., Lee, S. J., Wu, L., Sarntinoranont, M., \& Xie, H. (2012). Refractive index measurement of acute rat brain tissue slices using optical coherence tomography. Optics Express, 20(2), 1084. https://doi.org/10.1364/OE.20.001084

Sun, W., Cornwell, A., Li, J., Peng, S., Osorio, M. J., Aalling, N., Wang, S., Benraiss, A., Lou, N., Goldman, S. A., \& Nedergaard, M. (2017). SOX9 Is an Astrocyte-Specific Nuclear Marker in the Adult Brain Outside the Neurogenic Regions. The Journal of Neuroscience, 37(17), 4493-4507. https://doi.org/10.1523/JNEUROSCI.3199-16.2017

Suzuki, A., Stern, S. A., Bozdagi, O., Huntley, G. W., Walker, R. H., Magistretti, P. J., \& Alberini, C. M. (2011). Astrocyte-Neuron Lactate Transport Is Required for Long-Term Memory Formation. Cell, 144(5), 810-823. https://doi.org/10.1016/j.cell.2011.02.018

Synaptic Systems. (2018). Protocol for immunohistochemistry - vibratome sections.

Tauer, U. (2002). Advantages and Risks of Multiphoton Microscopy in Physiology. Experimental Physiology, 87(6), 709-714. https://doi.org/10.1113/eph8702464

Tavares, G., Martins, M., Correia, J. S., Sardinha, V. M., Guerra-Gomes, S., das Neves, S. P., Marques, F., Sousa, N., \& Oliveira, J. F. (2017). Employing an open-source tool to assess astrocyte tridimensional structure. Brain Structure and Function, 222(4), 1989-1999. https://doi.org/10.1007/s00429-016$1316-8$ 
Tønnesen, J., Inavalli, V. V. G. K., \& Nägerl, U. V. (2018). Super-Resolution Imaging of the Extracellular Space in Living Brain Tissue. Cell, 172(5), 1108-1121.e15. https://doi.org/10.1016/j.cell.2018.02.007

Tsai, P. S., Kaufhold, J. P., Blinder, P., Friedman, B., Drew, P. J., Karten, H. J., Lyden, P. D., \& Kleinfeld, D. (2009). Correlations of Neuronal and Microvascular Densities in Murine Cortex Revealed by Direct Counting and Colocalization of Nuclei and Vessels. Journal of Neuroscience, 29(46), 1455314570. https://doi.org/10.1523/JNEUROSCI.3287-09.2009

Uhlirova, H., Kılıç, K., Tian, P., Thunemann, M., Desjardins, M., Saisan, P. A., Sakadžić, S., Ness, T. V., Mateo, C., Cheng, Q., Weldy, K. L., Razoux, F., Vandenberghe, M., Cremonesi, J. A., Ferri, C. G. L., Nizar, K., Sridhar, V. B., Steed, T. C., Abashin, M., ... Devor, A. (2016). Cell type specificity of neurovascular coupling in cerebral cortex. ELife, 5(MAY2016), 1-23. https://doi.org/10.7554/eLife.14315

Valm, A. M., Oldenbourg, R., \& Borisy, G. G. (2016). Multiplexed Spectral Imaging of 120 Different Fluorescent Labels. 1-17. https://doi.org/10.1371/journal.pone.0158495

Vercelli, A. E., Garbossa, D., Curtetti, R., \& Innocenti, G. M. (2004). Somatodendritic minicolumns of output neurons in the rat visual cortex. 20(May), 495-502. https://doi.org/10.1111/j.14609568.2004.03483.x

Verveer, Hanley, Verbeek, Van Vliet, \& Jovin. (1998). Theory of confocal fluorescence imaging in the programmable array microscope (PAM). Journal of Microscopy, 189(3), 192-198. https://doi.org/10.1046/j.1365-2818.1998.00336.x

Villaseñor, R., \& Collin, L. (2017). High-resolution Confocal Imaging of the Blood-brain Barrier: Imaging, 3D Reconstruction, and Quantification of Transcytosis. Journal of Visualized Experiments, 2017(129), 1-9. https://doi.org/10.3791/56407

Weissman, T. A., \& Pan, Y. A. (2015). Brainbow: New Resources and Emerging Biological Applications for Multicolor Genetic Labeling and Analysis. Genetics, 199(2), 293-306. https://doi.org/10.1534/genetics.114.172510

Wen, J., Goyal, M. S., Astafiev, S. V., Raichle, M. E., \& Yablonskiy, D. A. (2018). Genetically defined cellular correlates of the baseline brain MRI signal. Proceedings of the National Academy of Sciences, 115(41), E9727-E9736. https://doi.org/10.1073/pnas.1808121115

Wilhelmsson, U., Bushong, E. A., Price, D. L., Smarr, B. L., Phung, V., Terada, M., Ellisman, M. H., \& Pekny, M. (2006). Redefining the concept of reactive astrocytes as cells that remain within their unique domains upon reaction to injury. Proceedings of the National Academy of Sciences, 103(46), 17513-17518. https://doi.org/10.1073/pnas.0602841103

Witcher, M. R., Park, Y. D., Lee, M. R., Sharma, S., Harris, K. M., \& Kirov, S. A. (2009). Threedimensional relationships between perisynaptic astroglia and human hippocampal synapses. Glia, 58(5), NA-NA. https://doi.org/10.1002/glia.20946

Wu, J., Guo, C., Chen, S., Jiang, T., He, Y., Ding, W., Yang, Z., Luo, Q., \& Gong, H. (2016). Direct 3D Analyses Reveal Barrel-Specific Vascular Distribution and Cross-Barrel Branching in the Mouse Barrel Cortex. Cerebral Cortex, 26(1), 23-31. https://doi.org/10.1093/cercor/bhu166

Wu, J., He, Y., Yang, Z., Guo, C., Luo, Q., Zhou, W., Chen, S., Li, A., Xiong, B., Jiang, T., \& Gong, H. (2014). NeuroImage 3D BrainCV : Simultaneous visualization and analysis of cells and capillaries in a whole mouse brain with one-micron voxel resolution. NeuroImage, 87, 199-208. https://doi.org/10.1016/j.neuroimage.2013.10.036 
Xiong, B., Li, A., Lou, Y., Chen, S., Long, B., Peng, J., Yang, Z., Xu, T., Yang, X., Li, X., Jiang, T., Luo, Q., \& Gong, H. (2017). Precise cerebral vascular atlas in stereotaxic coordinates of whole mouse brain. Frontiers in Neuroanatomy, 11 (December), 1-17. https://doi.org/10.3389/fnana.2017.00128

Xu, X., Cheng, J., Witt, R. M., Sabatini, B. L., \& Wong, S. T. C. (2006). A shape analysis method to detect dendritic spine in 3D optical microscopy image. 2006 3rd IEEE International Symposium on Biomedical Imaging: From Nano to Macro - Proceedings, 2006(February 2014), 554-557. https://doi.org/10.1109/isbi.2006.1624976

Xue, Q., Liu, Y., Qi, H., Ma, Q., Xu, L., Chen, W., Chen, G., \& Xu, X. (2013). A Novel Brain Neurovascular Unit Model with Neurons, Astrocytes and Microvascular Endothelial Cells of Rat. International Journal of Biological Sciences, 9(2), 174-189. https://doi.org/10.7150/ijbs.5115

Yamawaki, N., Borges, K., Suter, B. A., Harris, K. D., \& Shepherd, G. M. G. (2014). A genuine layer 4 in motor cortex with prototypical synaptic circuit connectivity. ELife, 3, e05422. https://doi.org/10.7554/eLife.05422

Yang, B., Treweek, J. B., Kulkarni, R. P., Deverman, B. E., Chen, C.-K., Lubeck, E., Shah, S., Cai, L., \& Gradinaru, V. (2014). Single-Cell Phenotyping within Transparent Intact Tissue through Whole-Body Clearing. Cell, 158(4), 945-958. https://doi.org/10.1016/j.cell.2014.07.017

Young, N. A., Collins, C. E., \& Kaas, J. H. (2013). Cell and neuron densities in the primary motor cortex of primates. Frontiers in Neural Circuits, 7(FEBRUARY 2013), 1-11. https://doi.org/10.3389/fncir.2013.00030

Zhang, L.-Y., Lin, P., Pan, J., Ma, Y., Wei, Z., Jiang, L., Wang, L., Song, Y., Wang, Y., Zhang, Z., Jin, K., Wang, Q., \& Yang, G.-Y. (2018). CLARITY for High-resolution Imaging and Quantification of Vasculature in the Whole Mouse Brain. Aging and Disease, 9(2), 262. https://doi.org/10.14336/AD.2017.0613

Zhang, X., Zhao, H., Zhang, S., \& Li, R. (2019). A Novel Deep Neural Network Model for Multi-Label Chronic Disease Prediction. Frontiers in Genetics, 10(APR), 1-11. https://doi.org/10.3389/fgene.2019.00351

Zhou, B., Zuo, Y., \& Jiang, R. (2019). Astrocyte morphology: Diversity, plasticity, and role in neurological diseases. CNS Neuroscience \& Therapeutics, 25(6), 665-673. https://doi.org/10.1111/cns.13123 
VITA

\section{JARED LEICHNER}

2006-2010

GPA: 3.6

2010-2011

GPA: 3.91

2011-2020

GPA: 3.93
B.A., Biochemistry \& B.A., Psychology

Harriet L. Wilkes Honors College of FAU

Jupiter, Florida

Presidential Excellence Award Finalist - 2020 FIU Recognition Awards

$1^{\text {st }}$ Place -2020 Graduate Research Day Oral Symposium

Finalist - 2019 3-Minute Thesis Competition

$1^{\text {st }}$ Place -2018 GSAW Oral Symposium

$1^{\text {st }}$ Place -2018 BMES Oral Symposium

FIU Technology Fee Grant Recipient - \$21,000

Kauffman Foundation Creative Project Grant Recipient - \$5,000

Betty G. Reader Scholarship Recipient \& Perry Graduate Fellowship Recipient

MBA, International Business

Florida International University

Miami, Florida

Voted 'Most Likely To Succeed' by Class of 2011.

2010 FIU College of Business Scholarship - $\$ 18,000$

Chapman Merit Fellowship Holder

Finalist in Intercollegiate Ethics Case Competition

Semi-Finalist of ACG Cup Case Competition

Participated in eight-week academic \& linguistic immersion in China

M.S., Biomedical Engineering \& PhD, Biomedical Engineering

Florida International University

Miami, Florida

Founders Honors Scholarship \& Golden Key Honor Society Member Participated in six-week academic (anthropological/linguistic) immersion in Peru/Ecuador (2007) \& repairs of New Orleans' Lower Ninth Ward with PeaceCorp and ACORN (2006).

\section{PUBLICATIONS}

Leichner, J, Lin, W. (2020). Advances in imaging and analysis of 4 fluorescent components through the rat cortical column. Journal of Neuroscience Methods.

Sarwar, M, Leichner, J, Naja, G, Li, C. (2019). Smart-phone, paper-based fluorescent sensor for ultra-low inorganic phosphate detection in environmental samples. Microsystems Nanoengineering.

Deshmukh, A, Leichner, J, Bae, J, Song, Y, Valdes, P, Lin, W, Riera, J. (2018). Histological characterization of the irritative zones in focal cortical dysplasia using a preclinical rat model. Frontiers in Cellular Neuroscience.

Leichner, J, Sarwar, M, Nilchian, A, Zhu, X, Liu, H, Shuang, S, Li, C. (2017). Electrochemical lateral flow paper strip for oxidative-stress induced DNA damage assessment.

Xizi Dai, Yen-Chih Huang, Jared Leichner, Madhvan Nair, Wei-Chiang Lin, Chen-Zhong Li. (2015) Peptide modified polymer poly (glycerol- dodecanedioate co-fumarate) for efficient control of motor neuron differentiation. Biomedical Materials.

Coarasa, A, Milera, A, Carvajal, D, Gulec, S, Leichner, J, McGoron, A. (2013). (68)Ga-NOTACHSg and (99m)Tc-CHSg Labeled Microspheres for Lung Perfusion and Liver Radiomicrospheres Therapy Planning. International Journal of Medical Imaging. 